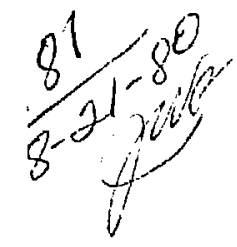

D. 1663

\title{
MASTER
}

UCRL-52868

\section{High-power pulsed lasers}

\author{
John F. Holzrichter
}

\section{April 2, 1980}

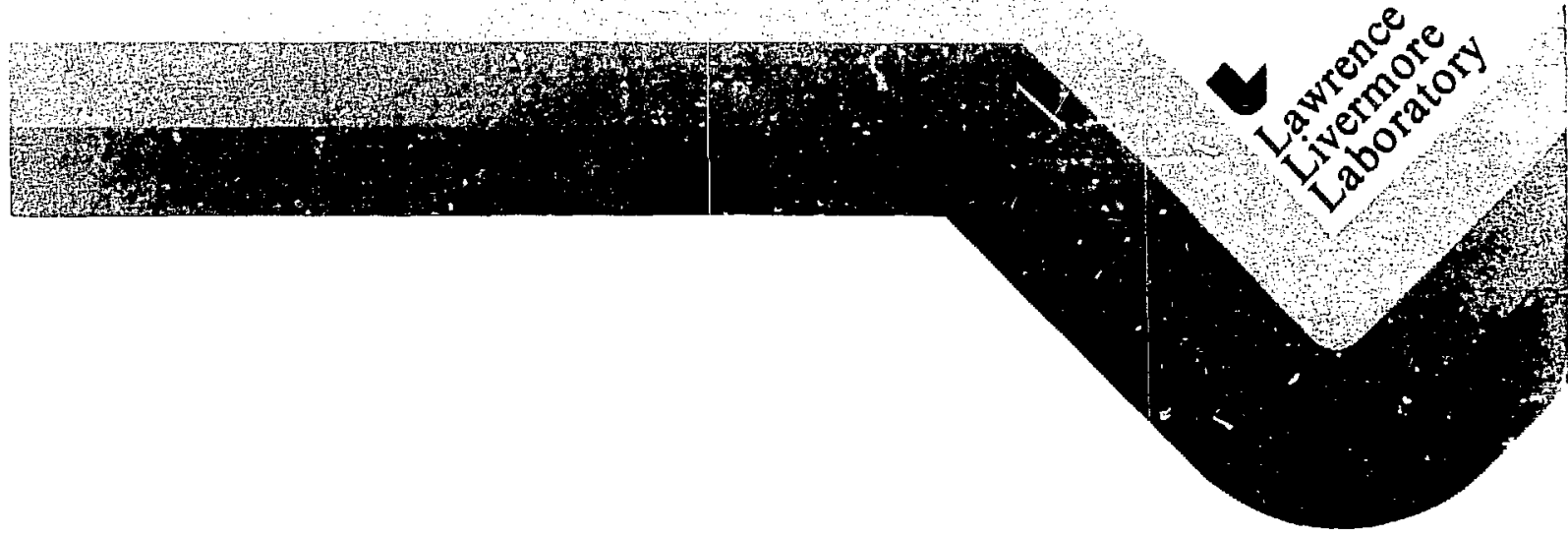




\title{
High-power pulsed lasers*
}

\author{
John F. Holzrichter \\ Manuscript date: April 2, 1980
}

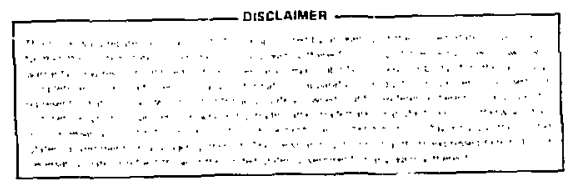

*A series of lectures for the 20th Scottish Iniversity Summer School in Physics, Laser Plasma Interaction

\section{LAWRENCE LIVERMORE LABORATORY University of California - Livermore, California - 14550}




\section{FOREWORD}

This report was originally presented as lectures for the $20 \mathrm{th}$ Scottish University Summer School in Physics on Laser Plasma Interaction, and is drawn fron many sources. Most of the contributions come from my colleagues in the Laser Program at the Lawrence Livermore Laboratory (LLL). Their voluminous output, summarized in the Laser Program Annual Reports from 1973 to the present (UCRL-500021-73 to -78), forms the basis for this work. Workers at the Naval Research Laboratory (Washington, D. C.), at Compagnie General d'Electricitie (Marcoussis, France), the University of Rochester (NY), the Lebedev Institute (Moscow), and the Los Alamos Scientific Laboratories (NM), have all made fundamental contributions to the technolegy of fusion lasers. I would like to acknowledge this influence on these lectures. In particular, I would like to acknowledge John L. Emmett, the Livermore Laser Fusion Program Director, for his encouragement and his innumerable contributions to the design of high-power fusion lasers. I would also like to thank Bill Krupke, who has led the advanced laser group at LLL. Most of the extensions of our laser design ideas beyond ND:glass are attributed to Bill and to his group. John Nuckolls deserves special mention for his work in target physics and its influence on laser design. Hal Ahlstrom's Fusion Experiment Program has made extensive contributions to target physics and to the optical diagnostics of lasers and targets.

In particular I would like to acknowledge my close working associates Thomas J. Gilmartin, Alexander J. Glass. Roger Haas and John B. Trenholme in the Laser Program for their assistance in this work. to Mrs. Patt Page for the preparation of the manuscript, to Mr. Robert Hendrickson for editorial assistance. and 1 extend my thanks to the members of the Fusion Program listed helow:

\section{l.aser design}

\section{J. A. Cilaze}

R. A. Haas

W. H. Hagen

W. W. Simmons

I B. Trenholme

\section{Propagation}

E. S. Bliss

A. J. Glass

J. T. Hunt

I. Seppala

W. W. Simmons

W. E. Warren

\section{Laser materials}

W. H. Lowdermilk

T. Marchı

D. Milam

W. L. Smith

S. F. Stokowski

E. P. Wallerstein

M. J. Weber

R. Wirtensen

\section{Laser engineering} and construction

L. Bradley

B. C. Johnson

D. J. Kuizenga

G. J. Linford

W. E. Martin

J. E. Murray

M. A. Summers

S. M. Yarema

\section{Diagnostics and alignment}

E. S. Bliss

F. W. Holloway

K. R. Manes

R. G. Ozarski

D. R. Speck

G. Suski

$J$ E. Swain

P. VanArsdall
W. L. Gagnon

T. J. Gilmartin

R. O. Godwin

C. A. Hurley

H. G. Patton

F. Reinecker

P. R. Rupert

I. F. Stowers

K. Whitham 


\section{CONTENTS}

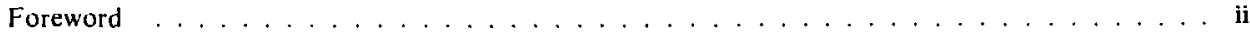

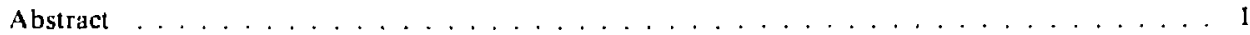

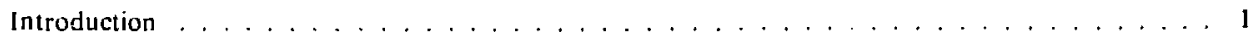

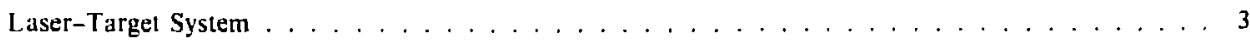

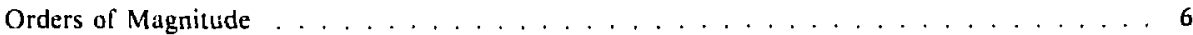

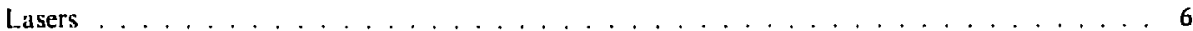

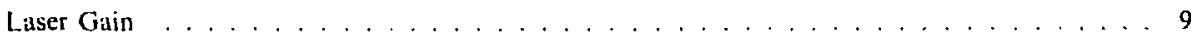

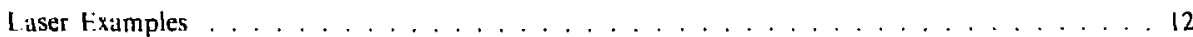

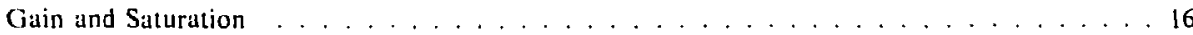

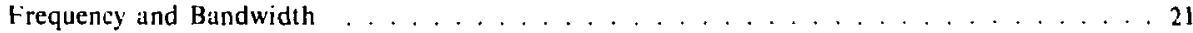

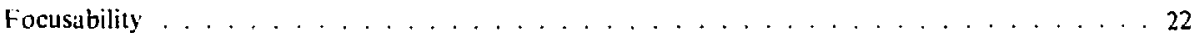

l.aser Operational constraints . . . . . . . . . . . . . . . . . . . . . 29

Gain Constraints . . . . . . . . . . . . . . . . . . . . . . . . 29

Chain Stability . . . . . . . . . . . . . . . . . . . . . . . . 29

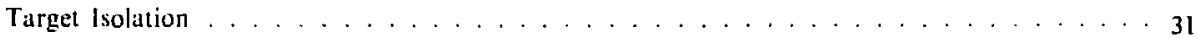

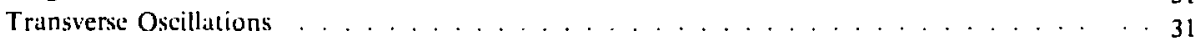

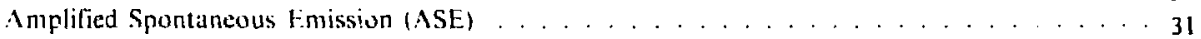

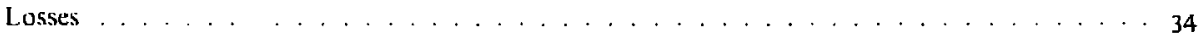

Nonlinear Optics . . . . . . . . . . . . . . . . . . . . . . . . . . 34

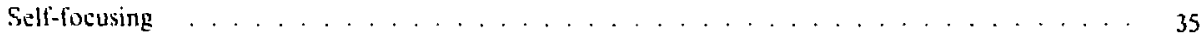

Damage limits . . . . . . . . . . . . . . . . . . . . . . . . 39

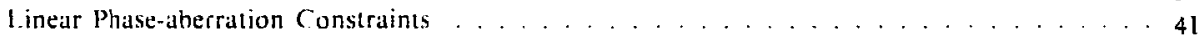

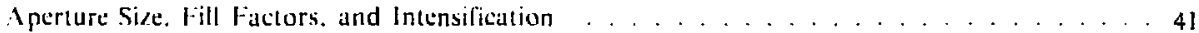

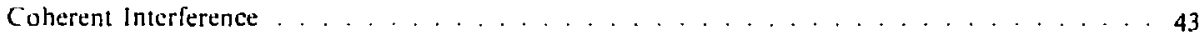

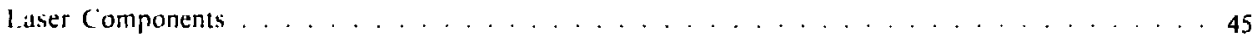

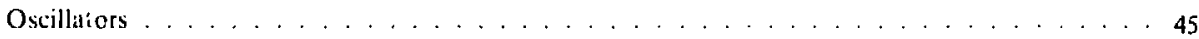

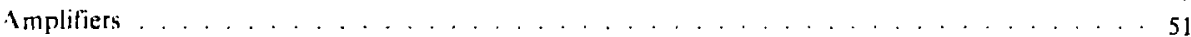

Vd:glass Medium . . . . . . . . . . . . . . . . . . . . . . . . . . 53

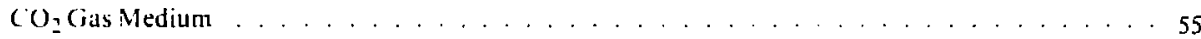

Spatial filters and Relays . . . . . . . . . . . . . . . . . . . . . . . 57

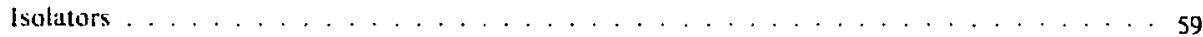

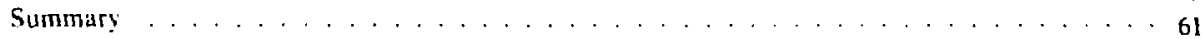

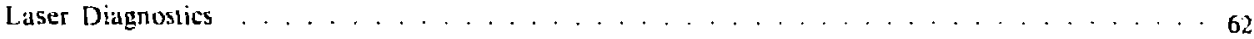

Vital Measurements . . . . . . . . . . . . . . . . . . . . 62

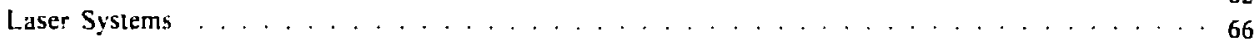

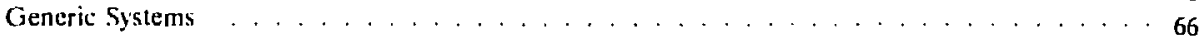

System Design . . . . . . . . . . . . . . . . . . . . . . . . . 68

Summary of Some Rules for Laser-stage Design . . . . . . . . . . . . . . . 70

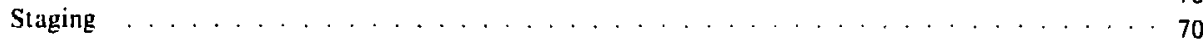

System Custs . . . . . . . . . . . . . . . . . . . . . . . . 74

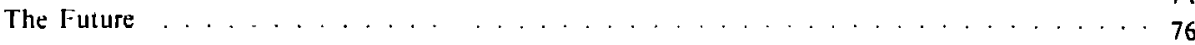

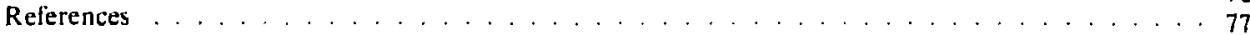




\section{High-power pulsed lasers}

\section{ABSTRACT}

The ideas that led to the successful construction and operation of large multibeam fusion lasers at the Lawrence Livermore Laboratory are reviewed. These lasers are based on the use of Nd:glass laser materials. However, most of the concepts are applicable to any laser being designed for fusion experimentation. This report is a summary of lectures given by the author at the 20 th Scottish University Summer School in Physics, on Laser Plasma Interaction. This report includes basic concepts of the laser plasma system, a discussion of lasers that are useful for short-pulse, high-power operation, laser design constraints, optical diagnostics, and system organization.

\section{INTRODUCTION}

With the demonstration of the laser in 1960 came the realization that its light could be focused and used to heat small but significant volumes of matter to temperatures high enough to induce fusion reactions. 1.2 In the laser, light is generated and propagated at low enough fluence levels so that optical damage to materials can be avoided. Most importantly, almost perfect coherence is retained, permitting focusing of the beam to areas of $\sim \lambda^{2}$ where $\lambda=$ laser wavelength. Thus, by focusing the laser output the power density on target can be increased enormously. Using this device, the experimenter can study matter under conditions of pressure. temperature. and density that have been attainable only under much more difficult experimental conditions such as in chemical explosions, hypervelocity gas guns, shock tubes, and nuclear explosions. For example, small targets $\left(\sim 10^{-7} \mathrm{~g}\right)$ are routinely heated to $1 \mathrm{keV}\left(10^{7} \mathrm{~K}\right)$ with $10 \mathrm{~J}, 0.1-\mathrm{ns}$ laser pulses. With a suitably intense laser beam and proper target construction, it was proposed that economical laser-induced nuclear fusion was feasible. ${ }^{3-5}$ This was followed by an experimental demonstration of laser fusion at KMS Fusion Inc.. Ann Arbor, M1, ${ }^{6}$ at LLL, ${ }^{7}$ and elsewhere. ${ }^{8}$

With the laser's invention in $1958,{ }^{9}$ national programs began, which have supported its development to the present, relatively well-understood experimental state. Some highlights for pulsed laser development are given in Table 1.

Much of the descriptive laser work presented here is based on laser science as developed in the 1960 s. Excellent references to the work of this period are given in Refs. 10 and 11. Glass lasers specific for plasma and fusion applications were developed in the mid-to-late 1960s by the Compagnie General d'Electricite (Marcoussis, France), at the Lebedev Institute (Moscow, Russia). at Sandia Laboratories (Albuquerque. NM), and at the Lawrence Livermore Laboratory (Livermore. CA). The power levels of the first systems were modest. about $0.01 \mathrm{TW}\left(1 \mathrm{TW}=10^{12} \mathrm{~W}\right)$. Since that time the technology of high-rower, pulsed-laser systems has developed dramatically. Examples are the 20-TW Shiva system (Fig. 1) and the Helios 10-TW $\mathrm{CO}_{2}$ laser. Now under construction are the Nova 300-TW glass laser and the Antares 100-TW $\mathrm{CO}_{2}$ laser. Designs for more energetic lasers $(\sim 1 \mathrm{MJ})$ have been proposed, with overall efficiencies of $\sim 5 \%$ and reliabilities of $>10^{7}$ shots between major maintenance cycles. A good compilation of articles on lasers for plasma applications is given in Ref. 12. A compendium of the Livermore work is given in the Livermore Laser Program Annual Reports. ${ }^{13}$ An excellent review of advanced laser concepts is given in Ref. 14.

This report was organized to give the reader an outline of the basic laser and optical principles that are important in high-power laser design. Examples of successes and failures are drawn primarily from experiences at the Livermore Laser Program. It is hoped that by reading this report. the reader will gain enough information on fusion-laser design that when the reader contemplates building a laser system. or simply using one for experiments, the system's limitations and possibilities will be apparent. 


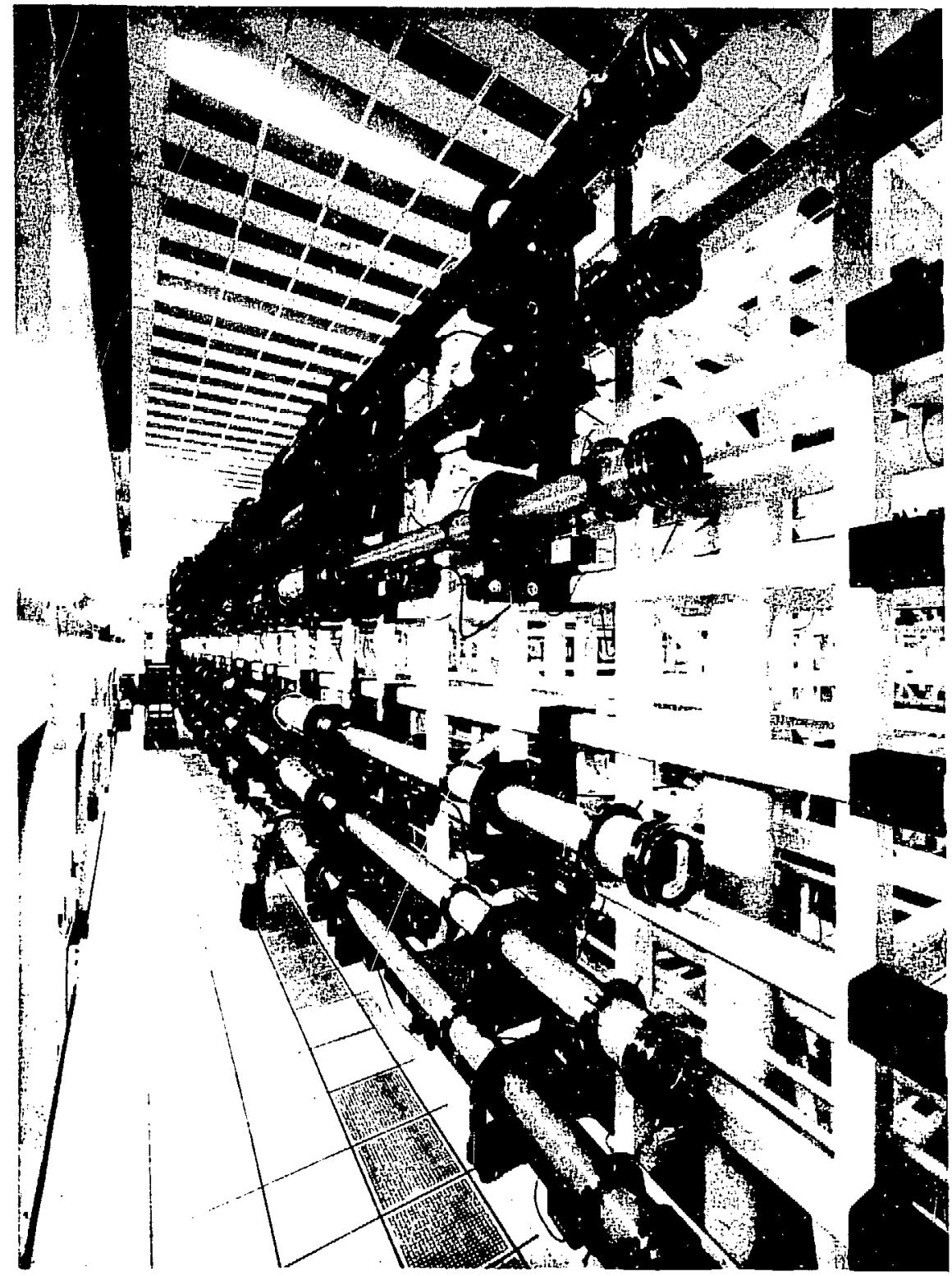

FIC. 1. Twenty-beam Shiva Laser system provides more than $20 \mathrm{TW}$ on a laser-fusion target in a $100 \mathrm{ps}$ pulse. 
TABLE 1. Pulsed laser developments.

\begin{tabular}{|c|c|c|}
\hline Devclopment & Investigators & Year \\
\hline Invention & Scliawlow and Townes & 1958 \\
\hline First laser $\left(\mathrm{Cr}^{3+}\right.$ in $\left.\mathrm{Al}_{2} \mathrm{O}_{3}\right)$ & Maiman & 1960 \\
\hline He-Ne laser & Javan, Bennett, and Herriott & 1961 \\
\hline Nd:glass laser & Snitzer & 1961 \\
\hline Master oscillator-pulse amplifier & Kisliuk and Boyle & 1961 \\
\hline Q-switching & Hellwarth & 1961 \\
\hline Giant pulse ruby laser & McClung and Hellwarth & 1962 \\
\hline $\mathrm{CO}_{2}$ laser & Patel & 1964 \\
\hline Nd: YAG laser & Geusic, Marios, and van Uitert & 1964 \\
\hline Iodine laser & Kasper and Pimentel & 1964 \\
\hline Faraday sotator & Aplet and Carion & 1964 \\
\hline Mode-locking & $\begin{array}{l}\text { DiDomenico } \\
\text { Hargrove et al. } \\
\text { Yariv }\end{array}$ & $\begin{array}{l}1964 \\
1964 \\
1965\end{array}$ \\
\hline Self-focusing & Chiau, Garmire, and Townes & 1965 \\
\hline Nd:glass fusion lasers & Ernest, Riffard, Robicux, and Sturel & $1965 / 66$ \\
\hline Disk laser amplifier & $\begin{array}{l}\text { Almasi et al. } \\
\text { Swain et al. }\end{array}$ & $\begin{array}{l}1966 \\
1969\end{array}$ \\
\hline TEA lasers & $\begin{array}{l}\text { Beaulieu } \\
\text { Dumanchin and Rocca-Serma }\end{array}$ & $\begin{array}{l}1969 \\
1969\end{array}$ \\
\hline Excimer laser & Basuv & 1970 \\
\hline Pulsed iodine laser & Hollis and Kompa & 1972 \\
\hline E-beam/sustainer & $\begin{array}{l}\text { Fenstermacher et al. } \\
\text { Daugherty et al. }\end{array}$ & $\begin{array}{l}1971 \\
1971\end{array}$ \\
\hline Uigh-power spatial fittering & Glaze, Rainer, and Simmons & 1975 \\
\hline KrF laser & Ewing and Brau & 1976 \\
\hline Relay imaging & Hunt & 1976 \\
\hline Sliva multi-bcam Nd:ylass iaser & Emmett et al. & 1977 \\
\hline Helios multi-beam $\mathrm{CO}_{2}$ laser & Boyer et al. & 1978 \\
\hline
\end{tabular}

\section{LASER-TARGET SYSTEM}

The laser-target inter،ction system consists of an oscillator, amplifiers, propagation devices, a target system. and diagnostics. The purpose of the laser-target system is to deposit sufficient energy in an appropriate time interval on interesting targets. In turn. the larget experimenter measures larget response as plasma temperature. material acceleration, $x$-ray production. and neutron production. To do this in a predictable fashion. or at least in a repeatable fashion. the laser input to the target must be well controlled. repeatable. and predictable. The experiment usually requires $10 \mathrm{~J}$ in $1 \mathrm{~ns}\left(10^{11)} \mathrm{W}\right)$ or greater in order to accomplish interesting physics. Although a great deal of interesting physics was accomplished with lasers of lower performance. modern laser-fusion-sriented experimentation is being carried out with devices exceeding this level. The present state of the art for laser systems is $>100 \mathrm{~kJ}$ in $1 \mathrm{~ns}$, or $>10^{14} \mathrm{~W}$. In fact. systems such as Shiva at L.L. are presently operating on target at $>2 \times 10^{13} \mathrm{~W}$ and Helios at Los Alamus Scientific Laboratory (L.ASL) is operating at $>2 \times 10^{12} \mathrm{~W}$,

Figure 2 shows the components of a laser system. They are (1) the oscillator. which creates the seed pulse with the proper pulse shupe: (2) the propagation path and telescope system, which projects and magnifies the beam as it leaves the oscillator and traverses the amplifiers and projects it to the alignment mirrors and focus lens: (3) the power amplifiers. which amplify the $0.1-1.0 \mathrm{~mJ}$ oscillator pulse to the desired level of $10^{1}-10^{5} \mathrm{~J}:(4)$ the system stabilizing and isolating elements. which prevent target reflections from returning and damaging the laser system and prevent the amplifier from self-oscillating off the target or its own surfaces: (5) the mirror and focusing lens system. which directs the laser light down onto the larget: (6) the turget itself. which defines the beam focal requirements: and (7) the diagnostic systems. which are used to analyze the performance of the laser and to determine the interactions of the laser beam with the larget.

Because laser oscillators must provide precisely controlled laser pulses. they are limited to $0.1-1.0 \mathrm{~mJ}$ of output energs. If the experiment requires $10^{1}-10^{5} \mathrm{~J}$ on target, the system putse gain will be at least $10^{4}-10^{8}$. 
Because of losses and saluration effects, the required smail-signal amplifier gain will be even larger $\left(10^{5}-10^{9}\right)$. I.aser amplifiers, which provide this gain in a ivell-controlled manner, come in convenient pulse-gain packages of 10-100X in smill diameters and 2-10X in large diameters. Approximately iu amplifiers in series are required to amplify the beam to reasonable levels. The diameter of the amplifier increases from approximately $1.11-50 \mathrm{~cm}$ in urder 10 accommodate the ever-increasing energy in the beam, while not exceeding damaging fluences.

The propagation elements expand and control the beam shape in such a way that no damage is done to the laser components by the intense laser field. These elements prevent the beam from hitting aperture cdges, provide correct diameter, etc., yet do this in such a fashion that they form the most spatiai ; compact, lawsil-cost laser chain. The isolator package is required to control both laser self-oscillation and target refleclion hecause, with ampitifier gains of $>10^{4}-10^{7}$. even the smallest dirt speck or target glint will provide al refleclivity of $1 \mathrm{O}^{-4}-1 \mathrm{O}^{-3}$, thus ciusing the laser-oscillation condition to be satisfied. If this oceurred, low-level flumescence noise would be amplified to large levels, causing laser self-damage or target destruction. Under pulse conditions, targets routinely reflect 10-20\% and sometimes more than $50 \%$. Amplification of this reflected light hack toward the laser, as the beam is "minified," will inevitably lead to damage. Thus, isolation is mandians!

The target system consists of the pointing mirrors, focusing lens, target manipulator, and diugnostic by nems. This sistem's primary purpose is to aim the laser beam (usually converging) onto a selected target areal. typically $100 \mu \mathrm{m}$ diam. At the same time the diagnostic system verilies the laser performance, i.e., it meitsures sthergy, pulse evolution, yuality of focal distribution. and energy reflected back from the target into the focusing lens. (The measurement of laser light scattered in uther directions will not he discussed here, a! hough wabl faser-energy atcounting is important because it is one of the few ways to accurately measure targei ithorption.) The most ohvious part of this system is the focus lens, which concentrates the bean energy (1) the larget. Howeser, the focil quality of this system ciannot overcome poor optical quality in the incoming liner heam. The licial quality is a function sf heam aberrations caused by thermal loading, nonlinear focusing, material index gradients and beam-induced material breakdown.

for mul largel experiments, and w ith reasonably designed lasers, the best anainable laser local-spot diambiter in usually unaller than reeded for the experiment. The experiment is often performed in the convergme wetion of the heam at the appropriate radius as shown in Figs. 3 and 4.

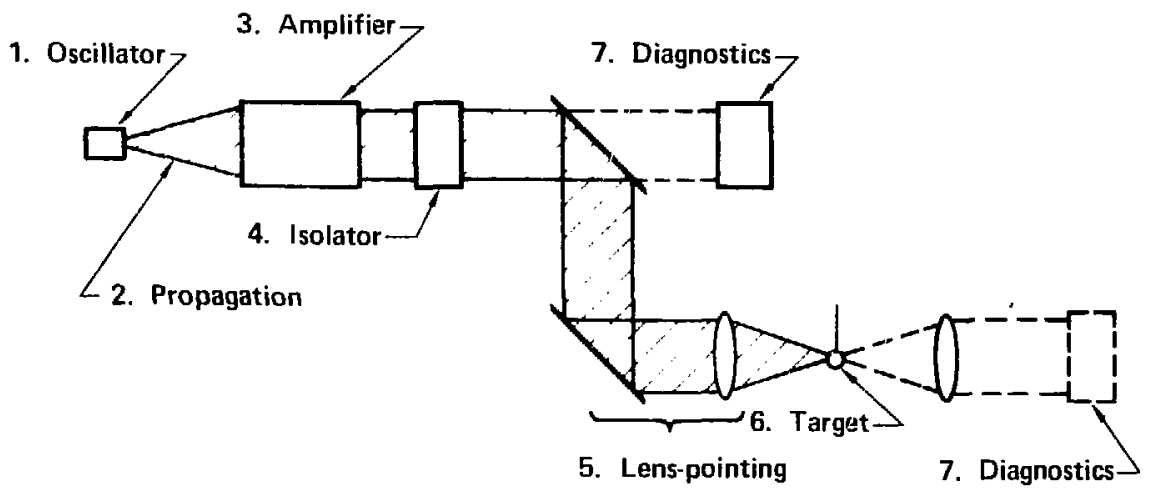

FIC. 2. Components in laser-target interaction system. 


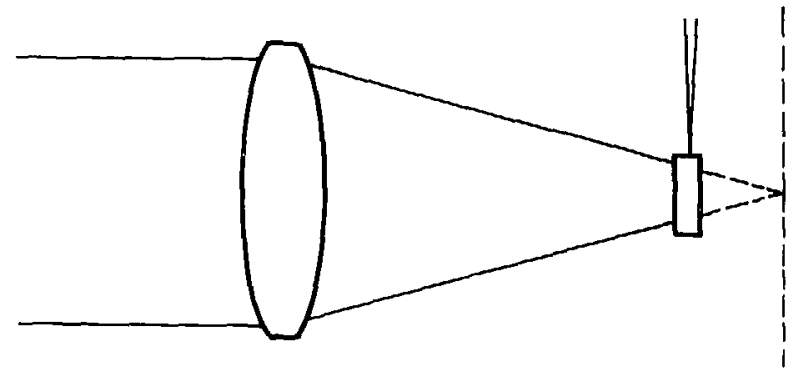

Focal plane

FIG. 3. Irradiated target is often located in front of the hest focus of the lens. Thus the experimenter controls power density and spot size. Foc.sl-spot quality is a function of beam's amplitude and phase uniformity.
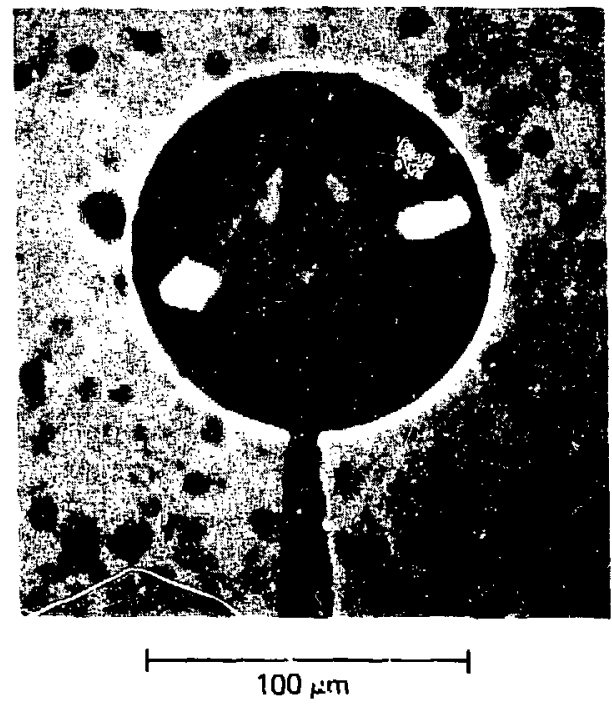

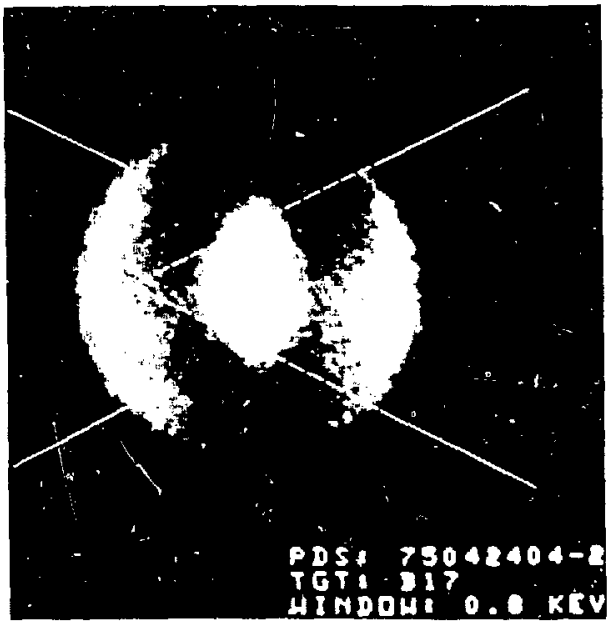

$800 \mathrm{eV}$ x-ray image

Target

FIG. 4. Simple ball target (left) is irradiated by two converging laser beams: (rigbt) $x$-ray image of the target implosion. ${ }^{13}$ 
TABLE 2. Orders of magnitude of measurements used in laser experiments.

\begin{tabular}{|c|c|}
\hline Measurement & Order of magnitude \\
\hline On-target energy & $10-10^{5} \mathrm{~J}$ \\
\hline $\begin{array}{l}\text { Pulse duration } \\
\text { (shorter pulses, } 5 \times 10^{-12} \mathrm{~s} \text {, are primarily } \\
\text { of interest as dingnostic tools) }\end{array}$ & $10^{-10}-10^{-8} \mathrm{~s}(0.1-10 \mathrm{~ns})$ \\
\hline $\begin{array}{l}\text { Average energy nuence } \\
\text { (governed by breakdown thresholds } \\
\text { and self-focusing) }\end{array}$ & $2-20 \mathrm{~J} / \mathrm{cm}^{2}$ \\
\hline Typical heam intensities & $10^{9}-10^{10} \mathrm{~W} / \mathrm{cm}^{2}$ \\
\hline $\begin{array}{l}\text { Peak beam electric fielus } \\
\text { (for } 10^{9}-10^{10} \mathrm{~W} / \mathrm{cm}^{2} \text { ) }\end{array}$ & $1-3 \times 10^{6} \mathrm{v} / \mathrm{cm}$ \\
\hline Peak beam mignetic fields & $3000-9000 \mathrm{C}$ \\
\hline Beam wavelengths & $\begin{array}{l}0.25 \times 10^{-4}-10^{-3} \mathrm{~cm} \\
(0.25 \mu \mathrm{m}-10 \mu \mathrm{m})\end{array}$ \\
\hline Beam frequencies & $3 \times 10^{13}-10^{15} \mathrm{~Hz}$ \\
\hline Optical periods & $\begin{array}{c}3 \times 10^{-14}-10^{-15} \mathrm{~s} \\
(0.03-0.001 \mathrm{ps})\end{array}$ \\
\hline Number of optical cycles in pulse of $1 \mathrm{~ns}$ & $3 \times 10^{4-10^{6}}$ \\
\hline Beam diameters & $5-50 \mathrm{~cm}$ \\
\hline Intensity on target & $10^{12}-10^{16} \mathrm{w} / \mathrm{cm}^{2}$ \\
\hline $\begin{array}{l}\text { Strength of the E ficlds for the above } \\
\text { focused spot }\end{array}$ & $3 \times 10^{7}-3 \times 10^{9} \mathrm{v} / \mathrm{cm}$ \\
\hline B fielus & $10 \mathrm{k}-10 \mathrm{MG}$ \\
\hline \multicolumn{2}{|l|}{ Typical time scales of interest } \\
\hline Laser optical period & $10^{-14} \mathrm{~s}$ \\
\hline Fusion bum time & $3 \times 10^{-11} \mathrm{~s}(30 \mathrm{ps})$ \\
\hline Lasti pulse durntion & $10^{-9} \mathrm{~s}(1 \mathrm{~ns})$ \\
\hline Laser medium pumping time & $10^{-7}-10^{-3} s$ \\
\hline Capacitor charging times & $1-10^{2} s$ \\
\hline
\end{tabular}

\section{ORDERS OF MAGNITUDE}

Table 2.

Several numbers are used routinely in laser-plasma experimentation. They are summarized in

\section{LASERS}

The laser described in Ref. 9 is a device that coherently amplifies optical radiation. (The acronym means Light Amplification by Stimulated Emission of Radiation.) The key observation leading to the demonstration of laser action was that certain nonthermal electron energy distributions could be created in materials, and under these conditions, the stimulated emission coefficient (the Einstein: B coefficient) associated with the electron transition will cause an optical field to grow in strength rather than be attenuated. By suitably placing an optical resonator (mirrors) around the prepared medium. spontaneous fluorescence associated with the randum de-excitation of the electron levels is collected by the surrounding mirrors (Fig. 5). It is redirected back and forth through the medium, and the optical field grows from the very low levels associated with spontaneous emissions to very high levels, which are usually limited by ma _rial breakdown. 


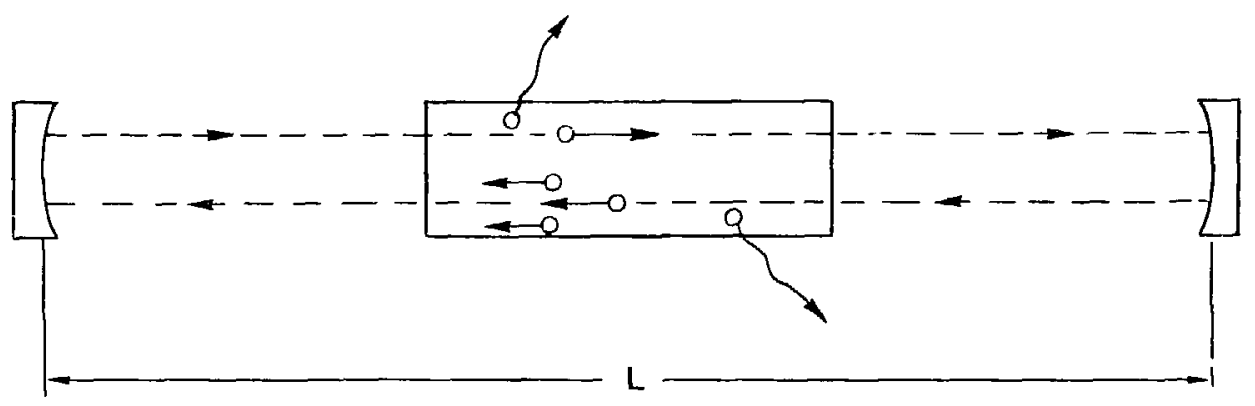

FIG. 5. Laser oscillation occurs when fluorescence noise grows in intensity in cavity.

As an example, select a 1 -cm-diam sphere from the laser rod in the cavity shown in Fig. 6. The rate of emission from the sphere of fluorescing material is approximated by dividing the instantaneous stored energy by the fluorescence decay time. For exämple, if we use a typical laser medium that stores $0.1 \mathrm{~J} / \mathrm{cm}^{3}$ and has a fluorescence decay time of $100 \mu \mathrm{s}$, then

$$
\frac{\mathrm{E}}{\mathrm{t}_{\mathrm{T}}}=\frac{\mathrm{C.} .1 \mathrm{~J} / \mathrm{sm}^{3} \times \mathrm{vol} .}{100 \mu \mathrm{s}}=522 \mathrm{~W} .
$$

Thus $522 \mathrm{~W}$ are radiated into $4 \pi$ sr of solid angle, through a surface area of $3.14 \mathrm{~cm}^{2}$. This leads to a 13-W/ $\mathrm{crn}^{2} / \mathrm{sr}$ fluorescence noise level.

This is now compared to the typical angular acceptance of a fusion target, which is $\mathrm{d} \theta \approx 10^{-4}$ rad, or a solid-angle acceptance of $(\mathrm{d} \theta)^{2}=\mathrm{d} \Omega=10^{-8} \mathrm{sr}$. In addition. lypical fluence limits for fusion lasers are $10^{9}-10^{10} \mathrm{~W} / \mathrm{cm}^{2}$. When beams of this fluence propagate within the $10^{-8} / \mathrm{sr}$ acceptance angle, $10^{17}-10^{18}$ $\mathrm{W} / \mathrm{cm}^{2} / \mathrm{sr}$ radiance levels must be achieved.

In Fig. 6 the noise level was $13 \mathrm{~W} / \mathrm{cm}^{2} / \mathrm{sr}$. Th: gain required to go from $13 \mathrm{~W} / \mathrm{cm}^{2} / \mathrm{sr}$ to $5 \times 10^{17} \mathrm{~W} / \mathrm{cm}^{2} / \mathrm{sr}$ is $38 \times 10^{16}$. If "gain-equal-to- 10 " amplifier sections are used in the laser, approximately

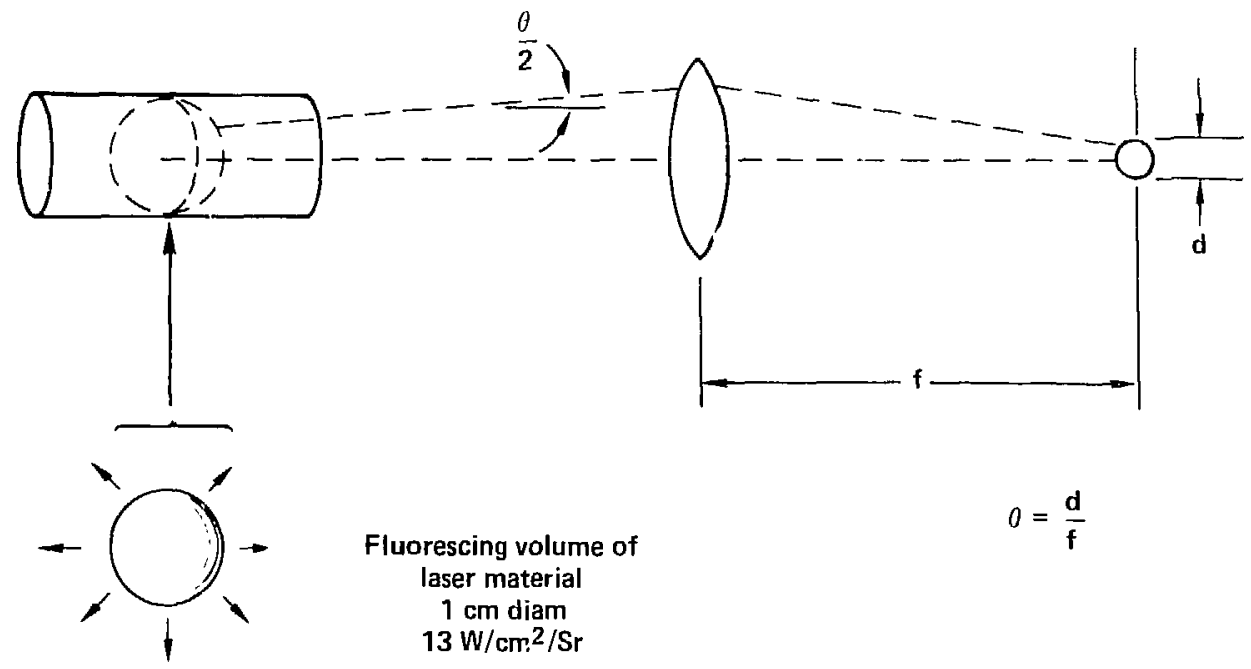

FIG. 6. Laser radiance is determined by power/area/solid angle. 


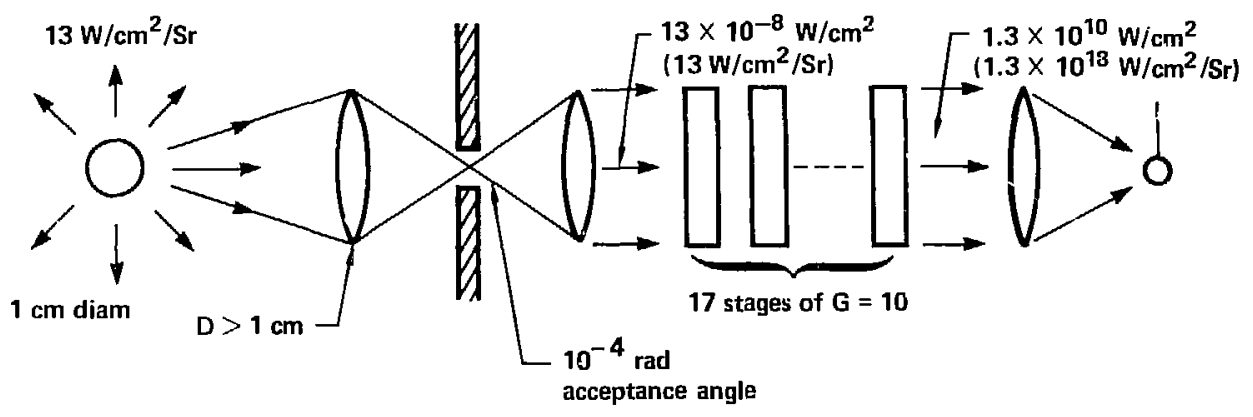

FIG. 7. Radiance amplification in laser system.

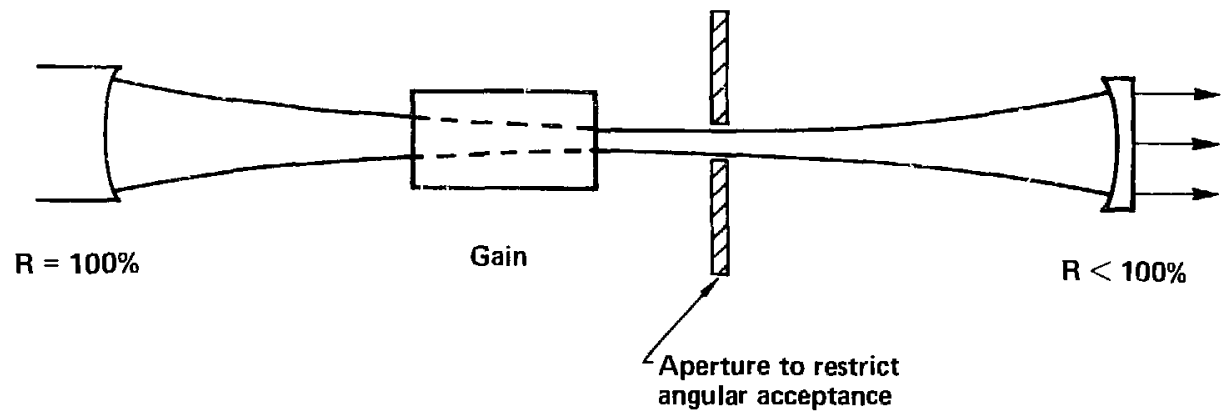

FIG. 8. Radiance amplification in cavity.

17 amplifiers are required to reach the required radiance levels (assuming no losses, aberrations, gain saturation, or area expansion). This is shown in Fig. 7.

The required radiance amplification can be performed in a very compact fashion (see Fig. 8) by placing mirrors around the gain medium and allowing the laser medium to both emit optical fluorescence noise to start the laser action, and to amplify the signal from the low-radiance levels of fluorescence noise to the high radiance required for target illumination. In other words, the oscillator cavity compactly amplifies the signal from noise to radiance levels limited by the beam-dianeter-imposed diffraction limit, by propagation constraints or damage, and/or nonlinear self-focusing limits. Stages of amplification following the oscillator can increase both the radiance of the oscillator pulse and the total beam power by reducing the diffraction-limited angular divergence, by increasing the area of the beam, and by amplifying the beam's intensity to the damage limit at the exit aperture of the amplifier system. The radiance and total beam power are eventually increased to the limit permitted either by dielectric breakdown or by defocusing optical aberrations associated with the increasing size of the amplifier system. An example ${ }^{15}$ of laser-beam radiance is shown in Fig. 9. 

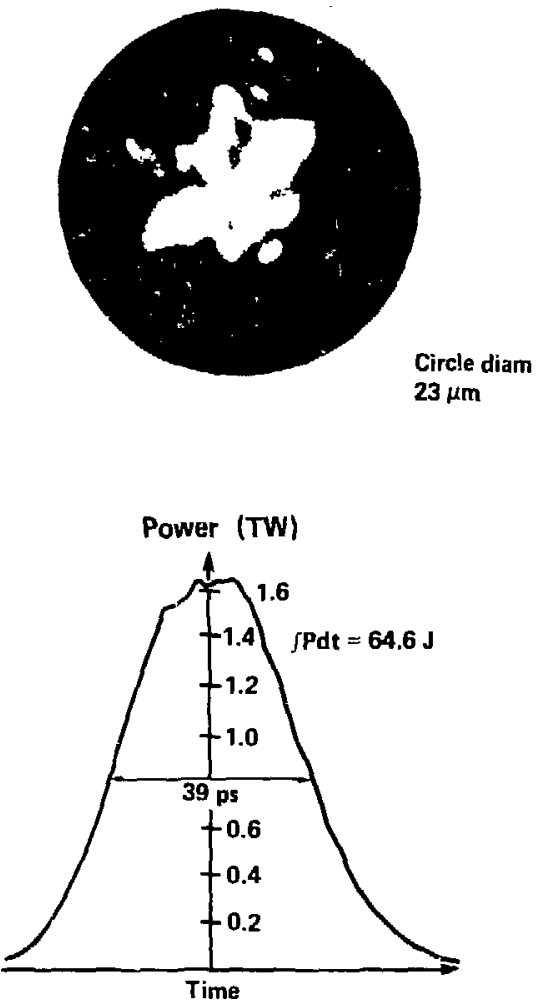

Shot $\# 4,9 / 30 / 76$
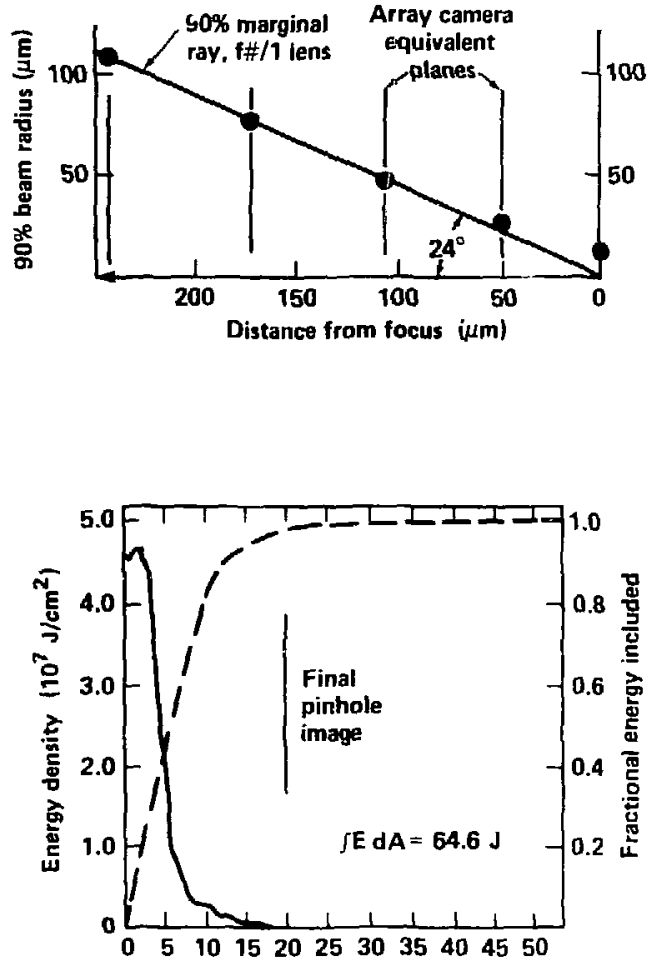

Radius from centroid $(\mu \mathrm{m})$ in plane of best focus

F1C. 9. Focal spot of Argus laser system. Radiance in this example is $1.5 \times 10^{18} \mathrm{~W} / \mathrm{cm}^{2} / \mathrm{sr}$.

\section{LASER GAIN}

The details of laser operation are given in several excellenı books and papers (e.g.. Re[s. 16 and 17 ) The amplification of a laser beam in a lossless medium proceeds as described by the following equation

$$
\frac{d I}{d z}=\alpha I
$$

where $\mathrm{I}$ is the laser energy fluence and $\alpha$ is the stimulated-emission coefficient. or the gain coefficient. Thi. equation integrates to

$$
I=I_{0} \exp (\alpha z) .
$$




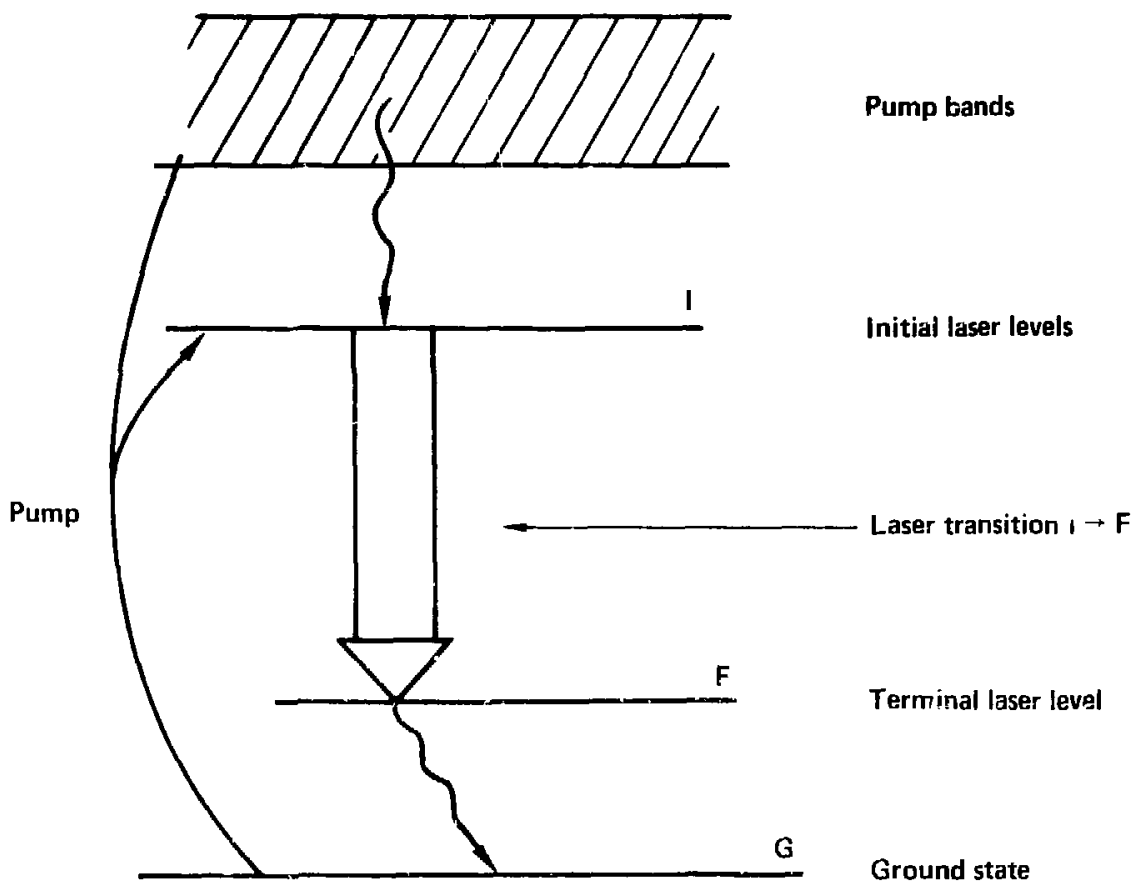

FIG. 10. Energy-level diagram for laser medium.

As long as $\alpha$ is independent of $\mathrm{l}$. the laser-beam fluence will exponentially increase as it traverses the gain medium. However, a does decrease as 1 increases, because the laser inversion level is decreased as the frint part of the optical pulse passes through the medium. leaving a lower gain for the rear part of the pulse.

The term $\mathrm{er}(\lambda)$ can be written as the sum of two parts, $a(\lambda)=\mathrm{N} \sigma(\lambda)$, where $\mathrm{N}$ is the inversion densit: in number per $\mathrm{cm}^{3}$ of the lasing species and $\sigma(\lambda)$ is the gain per ion, or the stimulated cross section. The units for $[\mathrm{N}]=\mathrm{cm}^{-3}$ and for $[\sigma(\lambda)]=\mathrm{cm}^{2}$.

A simple schematic of the optical transitions leading to laser gain is shown in Fig. 10.

Following notations in Ref. 17, the baser-gatin eoefficient at the center wavelength of the transition ss:

$$
\alpha(\lambda)=\left(N_{i}-\frac{B_{i}}{g_{j}} N_{f}\right) \lambda^{4} f(\lambda) / 8 \pi n^{2} t_{s} c
$$

At the line center wavelength for a I orenteian linewidth function, for example, pressure line broadening, the gain coefficient is

$$
\alpha(\lambda)=\left(N_{i}-\frac{B_{i}}{g_{f}} N_{f}\right) \lambda^{4} /(2 \pi)^{2} n^{2}+t_{s} \lambda \lambda .
$$


The terms in the gain equation, which vary with laser type, are as follows:

$\lambda \quad$ The wavelength, which varies rapidly with laser species.

$1 / t$ The spontaneous emission rate. which varies rapidly with the stimulatedemission matrix element. Short-wavelength lasers usually do not store energy as well as longer-wavelength lasers because their iluorescence lifetime is shorl and consequently their gain is high because $t_{s}^{-1}$ is large.

$N=\left(N_{i}-\frac{g_{i}}{g_{i}} N_{i}\right)$

The inversion-number density, which corresponds to energy storage in the medium. It varies between $10-500 \mathrm{~J} /$ /jter. The g's are degeneracy values describing the detailed atomic iransitions. The number $N$ is varied by adjusting the pump rate. This number should be as large as possible to reduce the volume of laser amplifier media.

n Index of ref ;ction of the "aser media.

id The laser line width, which varies strongly with the laser transition. Typical values $d$ re $300 \mathrm{~cm}^{-1}$ in $\mathrm{Nd}$ :glass to $<0.1 \mathrm{~cm}^{-1}$ for low pressure $\mathrm{CO}_{2}$. This parameter can be controlled to an extent by varying the pressure in a gas medium or by chousing different solid host materials to affect the characieristic ion line width.

$f(\lambda) \quad$ The normalized line-width function, $i_{-\infty}^{\infty} f(\lambda) d \lambda=i$, which measures the transition strength relative to the line peak as a function of $\lambda$.

As an example. consider a Nd:glass laser amplifier that stures $\sim 0.5 \mathrm{~J} / \mathrm{cm}^{3}$ in an inversion (belween the ${ }^{4} F_{3}{ }_{2}{ }^{-1} I_{11}$ 2 transition of $\mathrm{Nd}$ in laser glass). This corresponds to $3 \times 10^{18}$ excitations $\mathrm{cm}^{-3}$. For $\lambda_{\text {peat. }}=1.061 \mu \mathrm{m}, \mathrm{n}=1.56$, and $\mathrm{t}_{\mathrm{s}}=359 \mu \mathrm{s} /$ branching ratio. The branching ratio is the fraction of total radiative probability associated with the single transition from ${ }^{4} F_{3} \rightarrow^{4} I_{11}$ : here it is 0.484 . Futting these values into the gain equation above leads to a gain coefficient of $-0.08 \mathrm{~cm}^{-1}$ or $8 \% / \mathrm{cm}$. Examples of typical laser medium parameters are given in Table 3.

TABLE 3. Typical laser medium parameters.

\begin{tabular}{|c|c|c|c|c|c|}
\hline Laser & $x(\mu \mathrm{m})$ & $\theta\left(\mathrm{cm}^{2}\right)$ & $N\left(\mathrm{~cm}^{-3}\right)$ & $\Delta \lambda\left(\mathrm{cm}^{-1}\right)$ & $t_{5}(s)$ \\
\hline No:glass & 1.06 & $2.7 \times 10^{-20}$ & $3 \times 10^{18}$ & 340 & $360 \times 10^{-6}$ \\
\hline $\mathrm{CO}_{2}^{\mathrm{a}}$ & 10.6 & $6 \times 10^{-19}$ & $1 \times 10^{18}$ & 0.33 & 4.7 \\
\hline $\mathbf{I}_{2}$ & 1.3 & $2 \times 10^{-19}$ & $1 \times 10^{17}$ & 0.56 & 0.13 \\
\hline KrF & 0.249 & $2.5 \times 10^{-16}$ & $4 \cdot 10^{14}$ & 400 & $6.7 \times 10^{-9}$ \\
\hline
\end{tabular}




\section{LASER EXAMPLES}

Figures 11-14 show laser parameters for four laser media, which are of primary interest to the fusion experimenter. They are $\mathrm{Nd}$ :glass, ${ }^{18-21} \mathrm{CO}_{2}{ }^{22-24}$ iodine ${ }^{25-29}$ and $\mathrm{KrF}{ }^{30-32}$
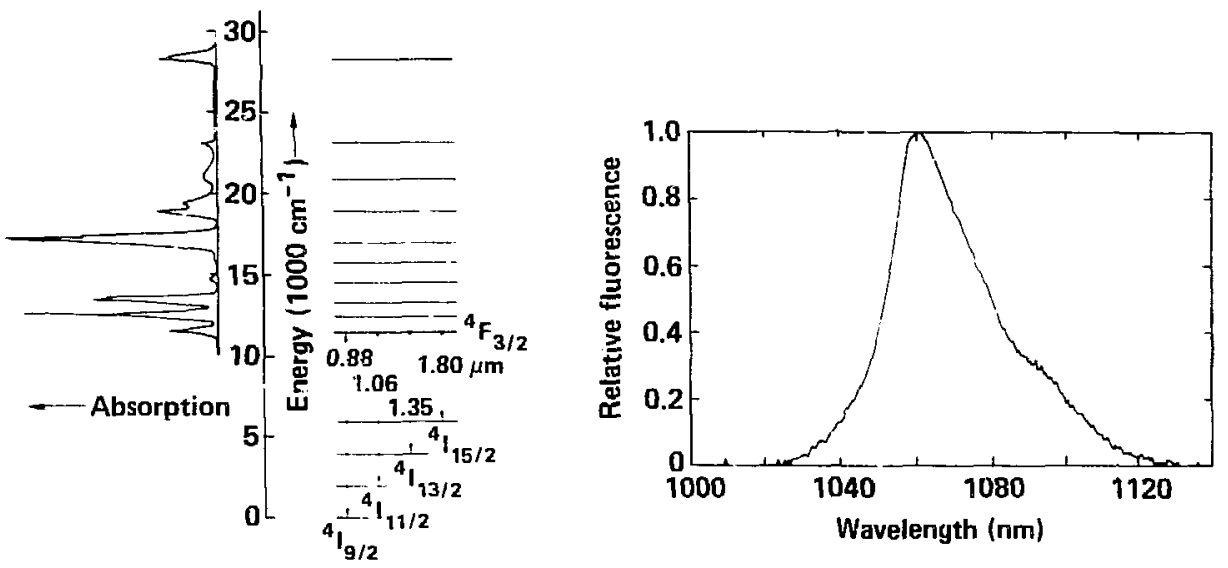

FIG. 11. Properties of Nd:glass laser.

Spectroscopic properties of $\mathrm{Nd}$ :

Wavelength

Cross section

Radiative lifetime

Fluorescence line width

Saturation fluence

$1061 \mathrm{~nm}$
$2.7 \times 10^{-20} \mathrm{~cm}^{2}$
$360 \mu \mathrm{s}$
$27.8 \mathrm{~nm}$
$5 \mathrm{~J} / \mathrm{cm}^{2}$.

Typicai laser amplifier parameters:

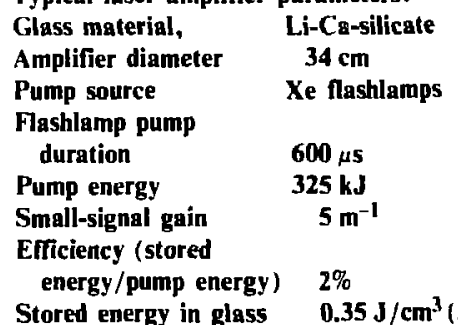

$0.35 \mathrm{~J} / \mathrm{cm}^{3}$ ( $350 \mathrm{~J} /$ liter ). 

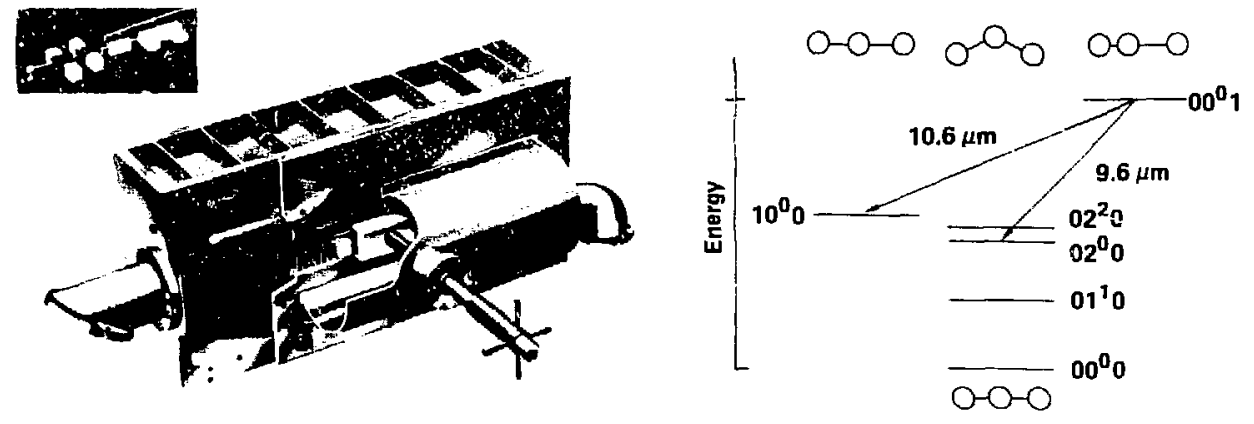

FIG. 12. Properties of the $\mathrm{CO}_{2}$ molecular laser.

Molecular properties:

Wavelength

Cross section

00"1 Radiative lifetime

$00^{0} 1$ Collisional lifetime (laser mix)

Saturation nuence

(all rot levels)
Typical laser purameters:

Gas mixture

Operating E/N

Applied field

Pump current density

Pump duration

Small-signal gain coefficient

Stored energy density

Efficiency (short pulse)

$$
\mathrm{He}: \mathrm{N}_{2}: \mathrm{CO}_{2} \text {, }
$$
1280:110:410 Torr

$\sim 2 \times 10^{-16} \mathrm{~V} / \mathrm{cm}^{2}$

$\sim 4 \mathrm{kV} / \mathrm{cm}$ atm

$-10 \mathrm{~A} / \mathrm{cm}^{2}$

2-4 us

$0.05 \mathrm{~cm}^{-1}$

$10-20 \mathrm{~J} /$ liter atm

2-10\%. 


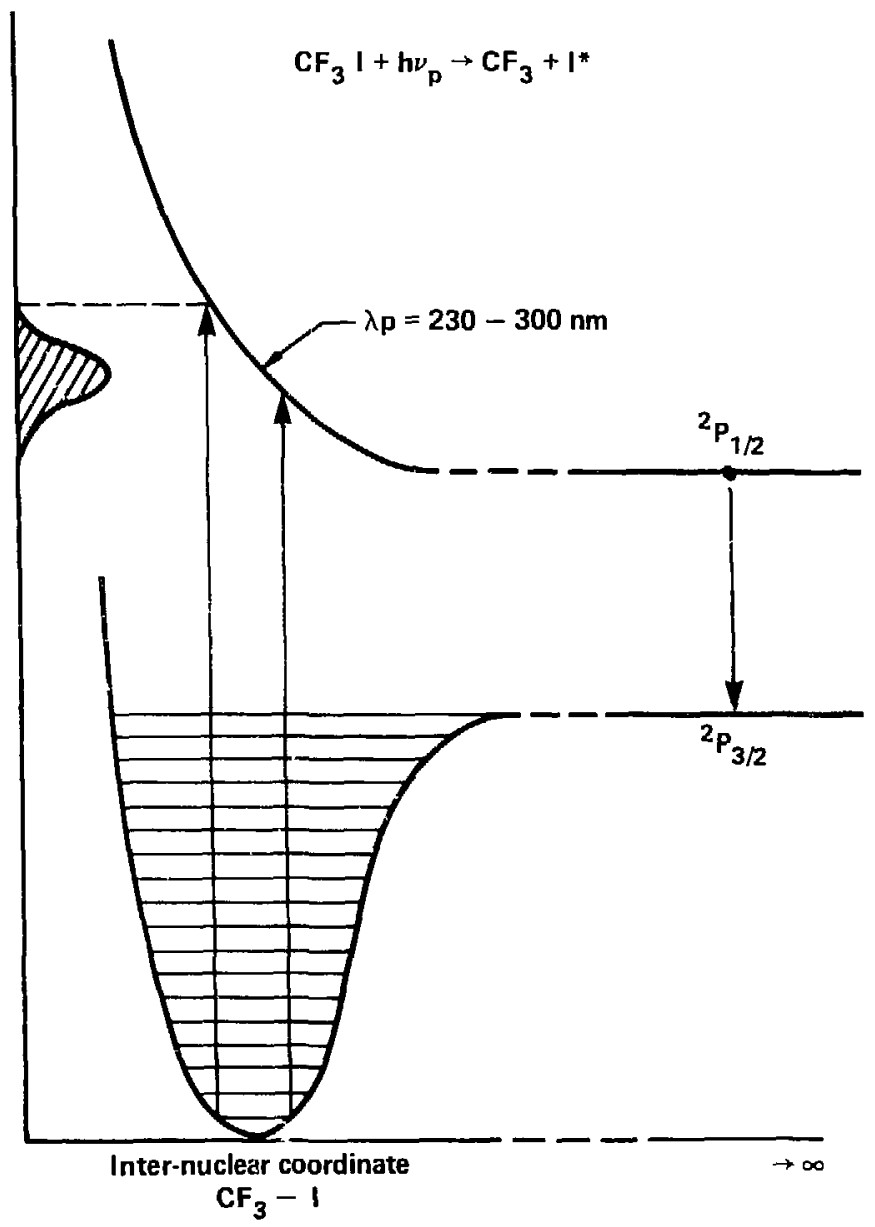

FIG. 13. Properties of the atomic iodine laser.

Atomic iodine properties:

Wavelength

Cross section

(laser mixture)

Radiative lifetime

Inversion lifetime

Saturation fuence

\section{$1.312 \mu \mathrm{m}$}

$2 \times 10^{-19} \mathrm{~cm}^{2}$

$130 \mathrm{~ms}$

$\sim 10 \mu \mathrm{s}$

$0.5 \mathrm{~J} / \mathrm{cm}^{-2}$.
Typical laser parameters:

Gas mixture

Pulse duration

Lamp fill

Small-signal gain coefficient

Stored inergy density

Efficiency
Ar: $\mathrm{C}_{3} \mathrm{~F}_{7} \mathrm{I}$ 1250:6 Torr

$10 \mu \mathrm{s}$

Xe, 5 Torr

$\sim 0.02 \mathrm{~cm}^{-1}$

$15 \mathrm{~J} /$ liter $\leqslant 1 \%$. 

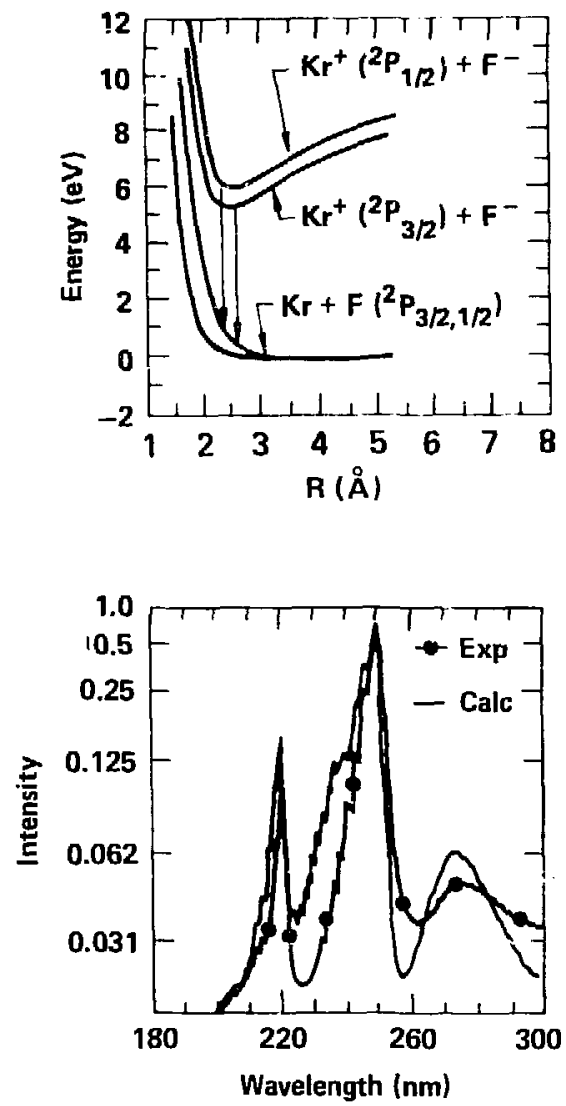

FiG. 14. Properties of the E-beam pumped KrF excimer laser.

Molecular properties:

Wavelength

Cross section

Radiative iffetime

Fluorescence line width

Saturation fluence

Saturation intensity

Formation kinetics:

$\mathbf{e}+\mathbf{F}_{2} \rightarrow \mathbf{F}^{-}+\mathbf{F}$

$\mathbf{e}+\mathbf{K r} \rightarrow \mathbf{K r}^{+}+2 \mathrm{e}$

$\mathbf{K r}{ }^{+}+\mathbf{K r}+\mathbf{A r} \rightarrow \mathrm{Kr}_{\mathbf{2}}^{+}+\mathbf{A r}$;

$\mathrm{Kr}_{\mathbf{2}}^{+}+\mathrm{Ar}+\mathbf{F}^{-} \rightarrow$

$\mathbf{K r F}+\mathbf{A r}+\mathbf{K r}$.

Typical laser parameters:

$249 \mathrm{~nm}$

$-2.5 \times 10^{-16} \mathrm{~cm}^{2}$

$\sim 6.7 \mathrm{~ns}$

$400 \mathrm{~cm}^{-1}$

$3 \mathrm{~mJ} / \mathrm{cm}^{2}$

$\geqslant 1 \mathrm{MW} / \mathrm{cm}^{2}$.
Gas mixtures

Beam voltage

Beam current

Pulse duration

Pamp rate

Sizull-signal gain

Nonsaturation photoabsorption loss coefficient

Gain/loss ratio

Nonsaturable photoabsorbers

Intrinsic efficiency
Ar:Kr:F, 712:46:2.3 Torr $\geqslant 200 \mathrm{keV}$

$10-30 \mathrm{~A} / \mathrm{cm}^{2}$

0.1-1 $\mu \mathrm{s}$

$0.3-1.0 \mathrm{MW} / \mathrm{cm}^{3}$

$0.01-0.1 \mathrm{~cm}^{-1}$

$\gamma \sim 5 \times 10^{-3} \mathrm{~cm}^{-1}$

$\alpha / \gamma$ 10-20

$F^{-}, F_{2}, A r_{2}^{\hbar}$

$10 \%$. 


\section{GAIN AND SATURATION}

In a saturating laser amplifier ${ }^{13-35}$ where the phase-memory time of the ions is short compared to the pulse width. and the pumping and decay times are long compared to the pulse width, the beam intensity of a pulse traveling toward $+z$ obeys

$$
\left.\dot{\partial I}+\frac{1}{\partial j} \frac{\partial I}{\partial t}=(\alpha-\gamma)\right\} \text {. }
$$

where $\mathrm{v}$ is the velocity of light in the medium (assuming there is no dispersion), $\alpha$ is the gain coefficient caused hy the inverted ions, and $\gamma$ is a nonsaturating fixed loss. The gain coefficient obeys

$$
d \omega=-\frac{\alpha I}{0} \text {. }
$$

Where", is a proportionality constant. We can see what this constant "s means by integrating Eq. (2) to obtain

$$
a=a_{11} \exp \left(\begin{array}{c}
f_{1 \mathrm{dt}} \\
0_{\mathrm{s}}
\end{array}\right) .
$$

The beam fluence at any point (energy per unit area) is defined as

$$
\partial(1)=\int_{-\infty}^{t} 1\left(1^{\circ}\right) d t^{\prime},
$$

so that Eq. (3) becomes

$$
\alpha=\alpha_{0} \mathrm{e}^{-\theta / \theta_{\mathrm{s}}}
$$

and we see that the fiuence " is that heam fuence which reduces the inverted population (and thus the g in coefficient) to I/c of its initial valuc, /, is generally called the saturation fluence.

It is convenient to change coordinates to a set in which

$$
r^{\prime}=\boldsymbol{\prime}
$$

and

$$
\mathrm{I}^{\prime}=1-1 / \mathrm{v}
$$

so that propagation moves the pulse in $z^{\prime}$ but not in $t^{\prime}$. We then have

$$
\frac{d l}{d z^{\prime}}=(\alpha-\gamma) l \text {. }
$$

which is integrable in time for the lossless case $(\gamma=0)$ to

$$
\frac{d \theta}{d z^{\prime}}=\int \alpha I d t^{\prime} .
$$

or using Eq. (4) for $\alpha$ :

$$
\begin{aligned}
\frac{\mathrm{d} \theta}{\mathrm{dz}} & =\int \alpha_{0} \mathrm{e}^{-\theta / \theta_{\mathrm{s}}} 1 \mathrm{dt} \mathrm{t}^{*} \\
& =\int \alpha_{0} \mathrm{e}^{-\theta / \theta_{\mathrm{s}}} \mathrm{d} \theta \\
& =\alpha_{0} \theta_{\mathrm{s}}\left(1-\mathrm{e}^{-\theta / \theta_{\mathrm{s}}}\right) \\
\frac{\mathrm{d} \theta}{\mathrm{d} \gamma^{\prime}} & =\frac{\alpha_{0} \theta_{\mathrm{s}}\left(\mathrm{e}^{\theta / \theta_{\mathrm{s}}}-1\right)}{\mathrm{e}^{\theta / \theta_{\mathrm{s}}}}
\end{aligned}
$$


With the change of variable

$$
u=e^{\theta / 0_{s}}-1 .
$$

we get from Eq. (6)

$$
\frac{\theta_{s}}{u+1} d u=\frac{\alpha_{0} \theta_{s} u}{u+1} d z^{\prime} .
$$

which is the same as

$$
\frac{d u}{u}=a_{0}^{\prime} d z^{\prime} .
$$

This integrates to

$$
u=u_{0} \mathrm{e}^{\int \alpha_{0} d x^{\prime}} .
$$

and if we recognize that

$$
\exp \left(\int \alpha_{0} d x^{\prime}\right)=G
$$

is the small-signal gain along the path of the pulse before saturation takes place, we arrive at the fundamental equation for saturated lossless gain

$$
e^{0 / \theta_{s}}-1=G\left(e^{0} 0^{/ \theta_{s}}-1\right) \text {. }
$$

relating output fluence $\theta$ to input fluence $\theta_{0}$, saturation fluence $\theta_{\mathrm{s}}$ and small-signal gain $\mathrm{G}$. This can be written as

$$
\theta=\theta_{\mathrm{s}} \ln \left[1+\mathrm{G}\left(\mathrm{e}^{0} 0^{1 / \theta_{\mathrm{s}}}-1\right)\right] .
$$

In the case where the lower laser level drains rapidly compared to characteristic pulse time... the saturation fluence is ${ }^{3}$

$$
\theta_{\mathrm{s}}=\frac{\underline{\underline{w}}}{\sigma} \text {. }
$$

where $\sigma$ is the cross section of the transition, $h$ is Planck's constant, and $\mu$ is i'.e frequency of the transition. If relaxation of the lower level is blocked, we have (where $k=g_{1} / g_{j}$ )

$$
\theta_{\mathrm{s}}=\frac{\mathrm{h} \nu}{\sigma(\mathrm{I}+\mathrm{k})} \text {. }
$$

where $k$ is the degeneracy ratio of the upper and lower levels. For a simple two level system. $k=1$ and the saturation fluence is half of the full-drain value. In the intermediate case where partial drain lakes place, we cannot use the equations we started with. However, experience has shown that satisfactory results may be obtained by the use of an "equivalent saturation fluence," which depends on the pulse duration.

Note that we may differentiate the fluence growth equation to get the intensity gain as a finction of time during the pulse as

$$
I\left(1^{\prime}\right)=\frac{I_{0}\left(t^{\prime}\right)}{1-\mathrm{e}^{-0_{0} / o_{\mathrm{s}}}(1-1 / \mathrm{G})} .
$$

where now $\theta_{\mathrm{c}}$ is the input fluence up to time $t^{\prime}$ in the pulse. This expression allows us to find the temporal distortion of saturated pulses. Some examples from Ref. 35 are shown in Figs. 15-18.

Up to this point we have ignored the effects of a fixed loss coefficient $\gamma$. Recalling that

$$
\frac{d I}{d l^{2}}=(\alpha-\gamma) I \text {. }
$$




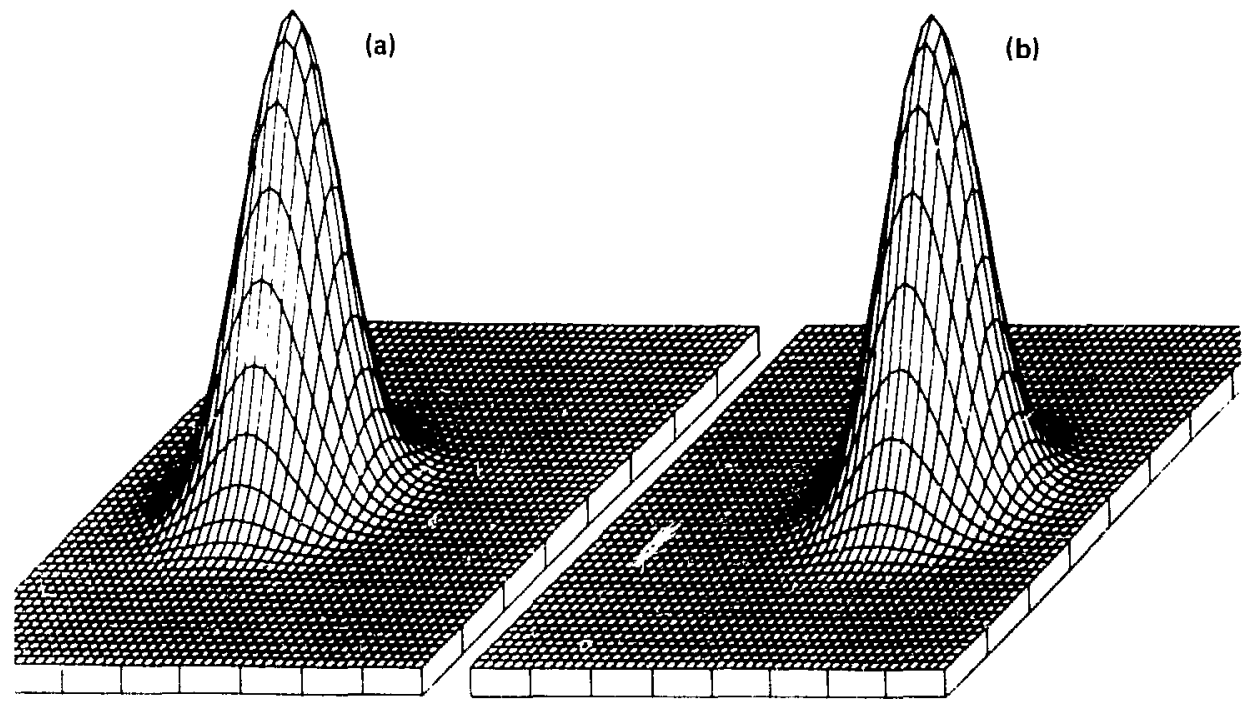

HIC. 15. Spatial and temporal shape of the initial pulse into a lossiess satyrating laser amplifier. The pulse is a spatial (front to back) and temporal (left to right) Gaussian with radial symmetry. (a) Front vien : (b) rear view.

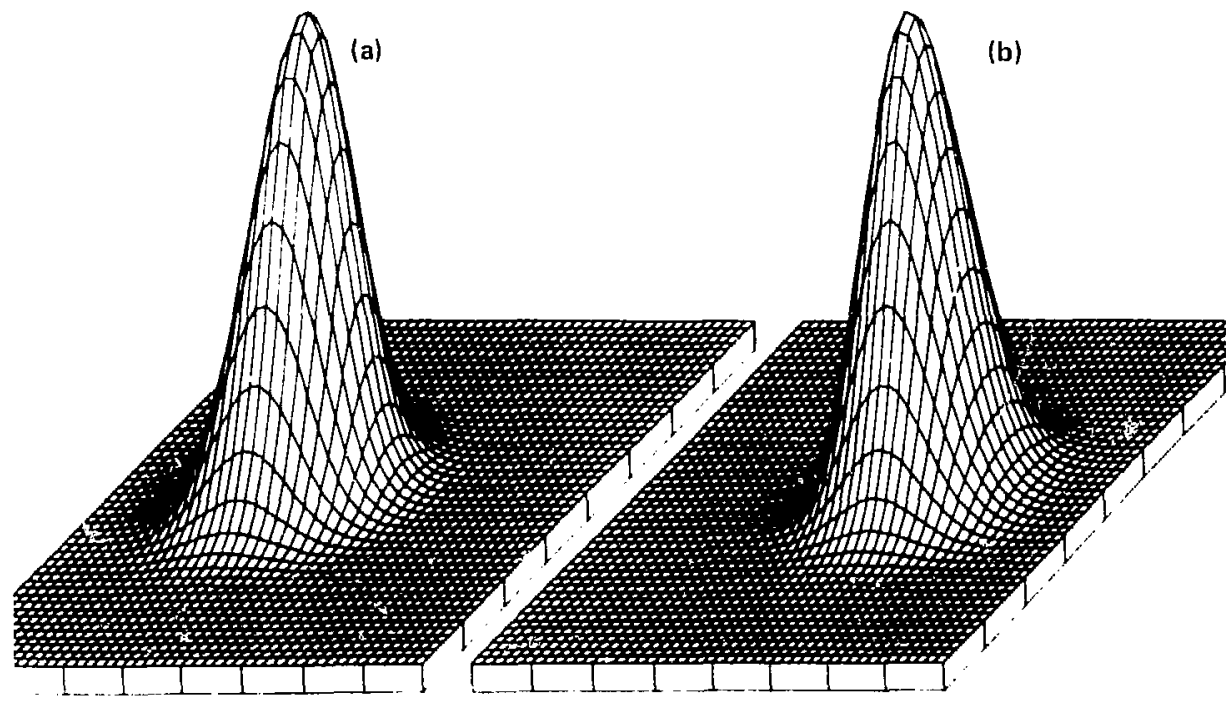

Fi. 16. Spatial and temporal shape of an initially Gaussian pulse in a lossless saturating laser amplifier. Finergy density at center is $i$ saturation fuence. (a) Front view: (b) rear view. (Time is left to right.) 


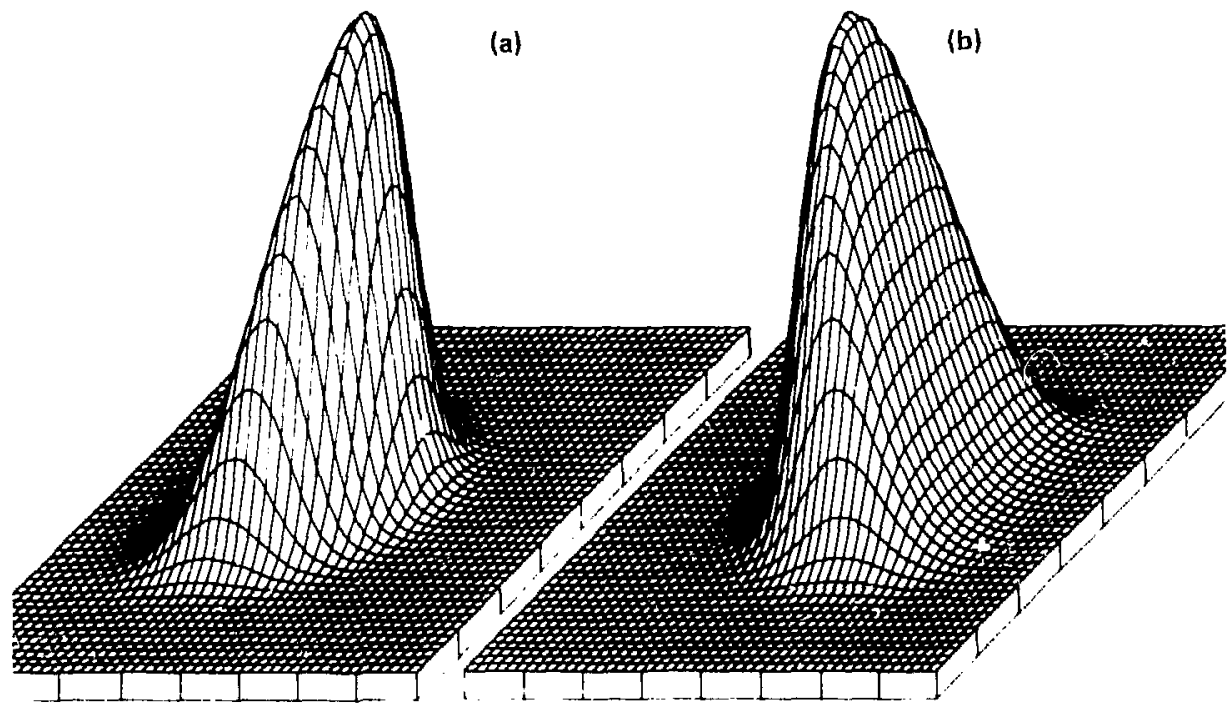

FIG. 17. Spatial and temporal shape of an initially Gaussian pulse in a lossless saturating laser amplifier. Energy density at center is $\mathbf{5}$ saturation fluences. (a) Front view; (b) rear view. (Time is left to right.)

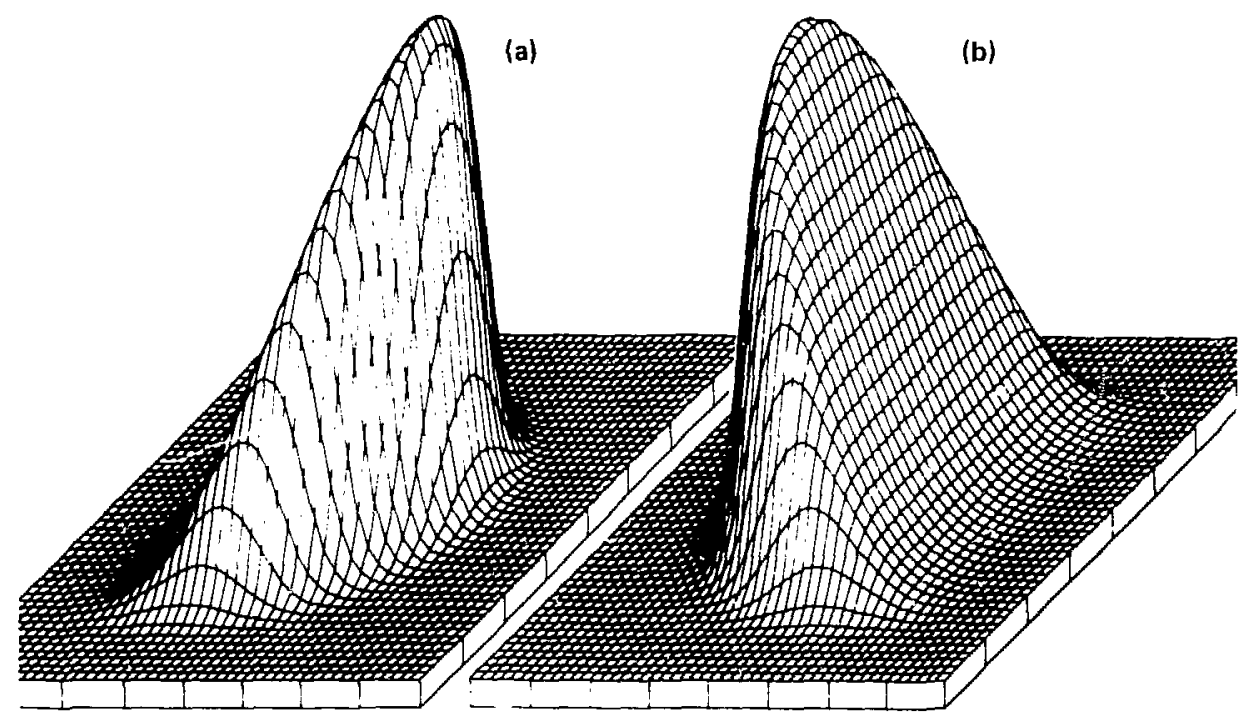

FIG. 18. Spatial and temporal shape of an initially Gaussian pulse in a lossless saturating laser amplifier. Energy density at center is 10 saturation fluences, (a) Front view; (b) rear view. (Time is left to right.) 
we see that for low-level signals the small-signal gain changes from

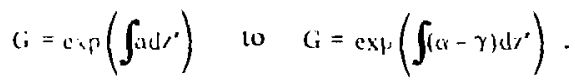

There must be enougi inversion that $\alpha>\gamma$ or the amplitier will be an allenuator. Even this is not enough if the beam saturates the amplifier ans red.ces $\alpha$ below $\gamma$. Including loss, we have from $\mathrm{Eq}$. (6):

$$
\frac{\mathrm{d} \theta}{\mathrm{d} \mathrm{z}^{\prime}}=a_{11} \theta\left(1-\mathrm{e}^{-1) / 0_{\mathrm{s}}}\right)-\gamma 0 \text {. }
$$

so the lluence ciannot rise above an equilibrium value given by

$$
\frac{d \theta}{d \%}=0 \quad \text { or } \quad \mathrm{e}^{-1)^{\prime} \omega_{\mathrm{s}}}=1-\frac{\gamma \theta}{\alpha_{0} 0_{\mathrm{s}}} \text {. }
$$

Some solutions $\left(\theta / \theta_{s}\right)$ of this equation for various ratios of gain to loss are:

$$
\begin{array}{ccccccc}
\alpha_{0} / \gamma & 1.1 & 1.2 & 1.5 & 2 & 5 & 10 \\
\hline 0 / 0_{5} & 0.1937 & 0.3764 & 0.8742 & 1.594 & 4.965 & 10
\end{array}
$$

The conclusion is that the beam fluence (neeasured in salturation fluences) cannot exced the ratio of gain 10 loss. The ahove analysis refers to latser pulses short compared to medium lifetimes. For laser pulses long conpilred to medium lifetimes, the equation in Fig. 19 holds. This observation is especially relevant to the KrF laser ststem where a nonsuturating loss is present, $0.005 \mathrm{~cm}^{-1}$, and the saturation flence is lou, $0.003 \mathrm{~J}: \mathrm{cm}^{2}$. Figure 19 shous at example ul this problem.
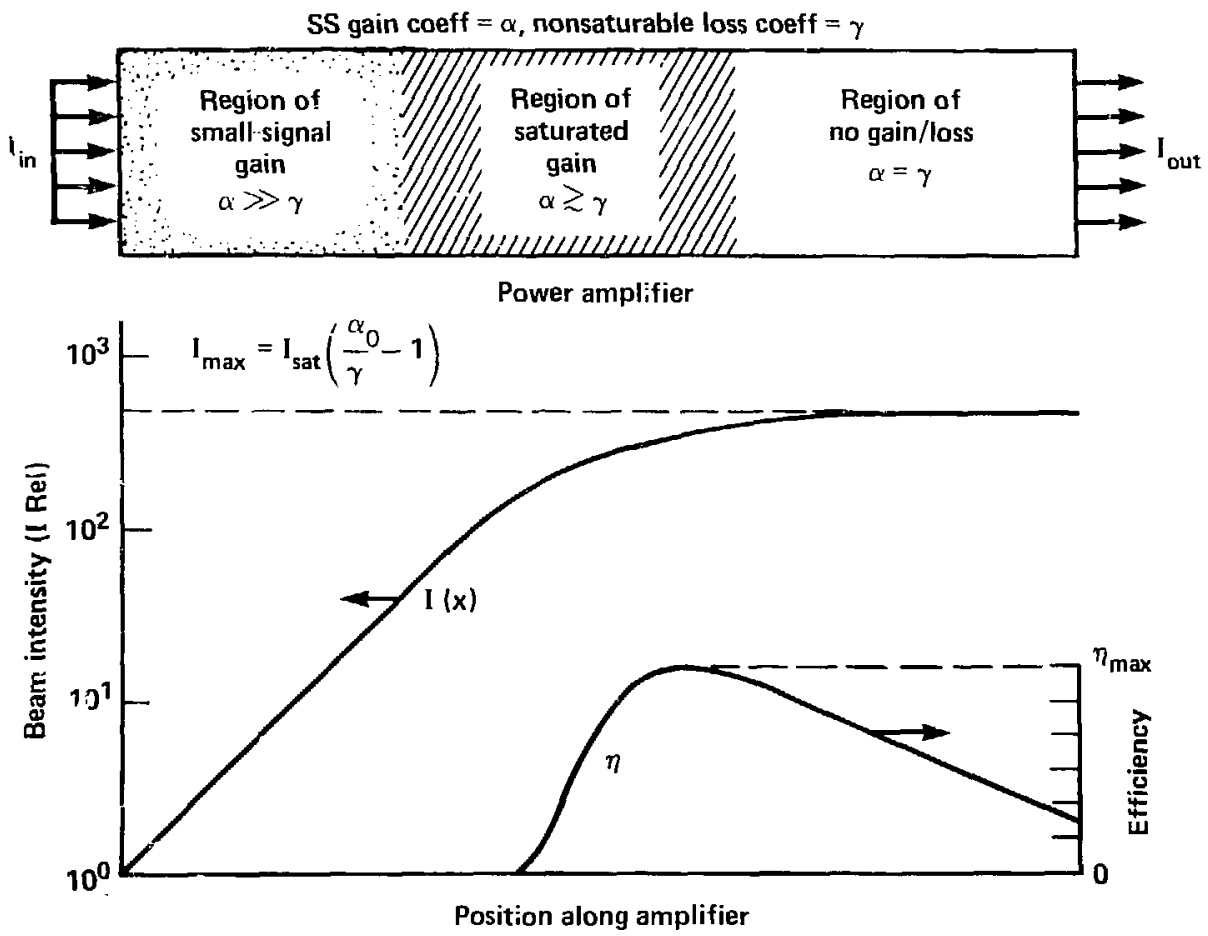

FIG. 19. Norsaturable losses in power amplifiers limir the maximum fluence to be $I_{\text {sat }}\left[\left(a_{0} / \gamma-1\right]\right.$. 


\section{FREQUENCY AND BANDWIDTH}

The laser gain is a function of frequency as described previously. The line-width function $f(\lambda)$ is determined by the shape of the laser transition lines. The shape is determined by a variety of physical processes-Doppler and collision effects in gases: strain or lifetime in solids.

The resultant line width of the laser signal is influenced by this line shape. because it determines both the fluorescence noise spectrum and the gain spectrum. This fluorescence line as measured by the FWHM value is narrowed by the laser gain. This is a consequence of the large $\left(\sim 10^{17}\right)$ gain required to develop the laser emittance from the spontaneous level to that of a fusion laser. As an example, consider a typical laser transition with a gain coefficient of $0.05 \mathrm{~cm}^{-1}$; that is, a small-signal gain $=e^{0.05 \%} \sim 10^{17}$.

The length of material traversed (both in a cavity and in the amplifie: chain) would be about $780 \mathrm{~cm}$ to reach the gain of $10^{17}$ if the lisser signal were at line center. Consider the gain at $\left(\lambda_{0}-\delta\right)$ in Fig. 20, where $\alpha\left(\lambda_{0}-\delta\right)=0.9 \alpha_{\max }=\left(0.045 \mathrm{~cm}^{-1}\right)$; then $\mathrm{e}^{(0.045) 780}=2 \times 10^{15}$, which is a factor of 50 lower than the gain at line peak. One finds that line widths are reduced by approximately $(\ln G)^{1} 2$ after a gain of $G$.

Figure 21 sketches the narrowing of the fluorescence line width as a consequence of the exponential gain process. If other frequency-selection elements are inserted in the oscillator cavity or in the amplifier chain, further spectral modification occurs. This is discussed later. Finally. while this example shows the results of exponential gain in the time domain, similar effects occur if amplifier gain is not uniform in space over the gain aperture. For example. a long series of rod amplifiers, each with small nonuniformities in gair. vs radius, can cumulatively have a very nonuniform gain vs radius profile.

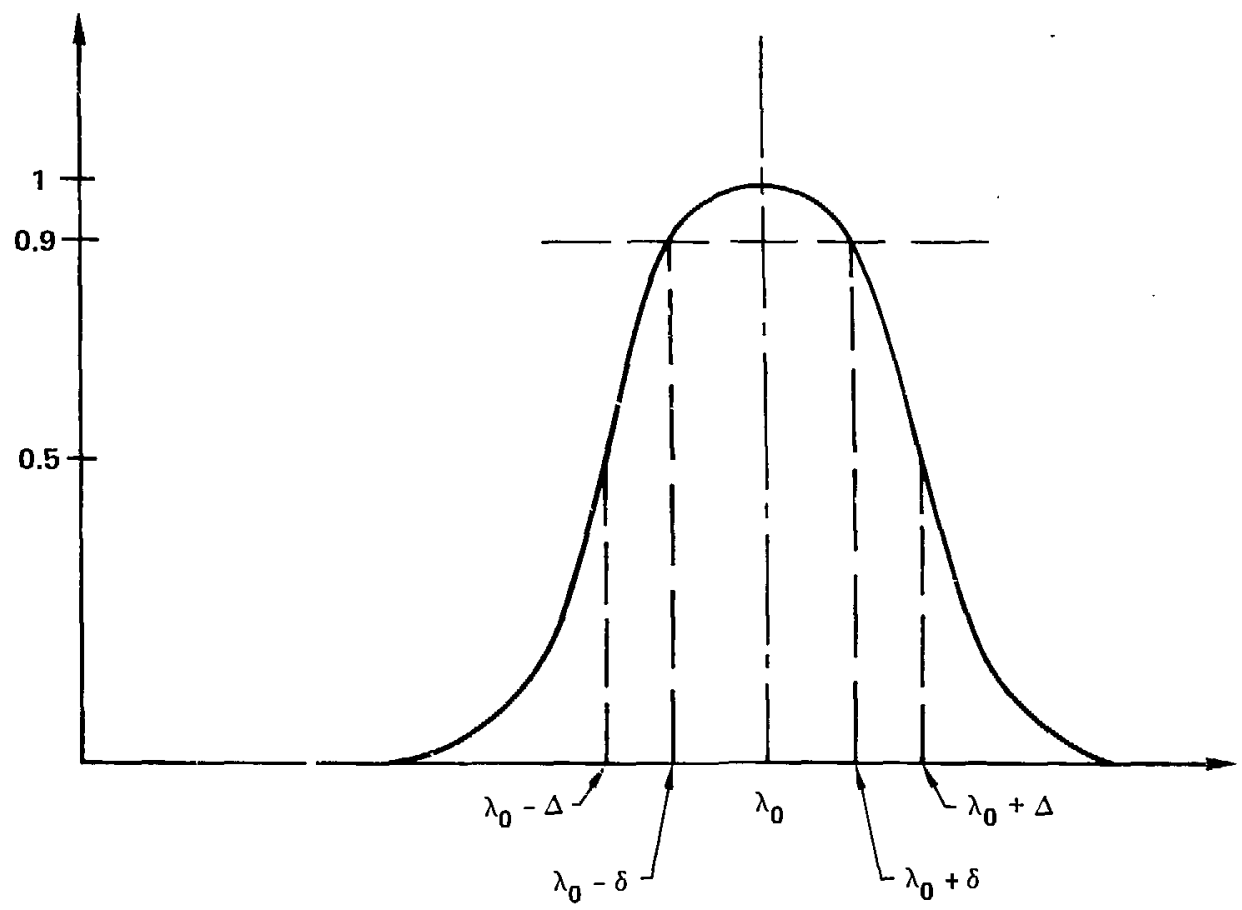

FIG. 20. Laser gain as a function of wavelength. 


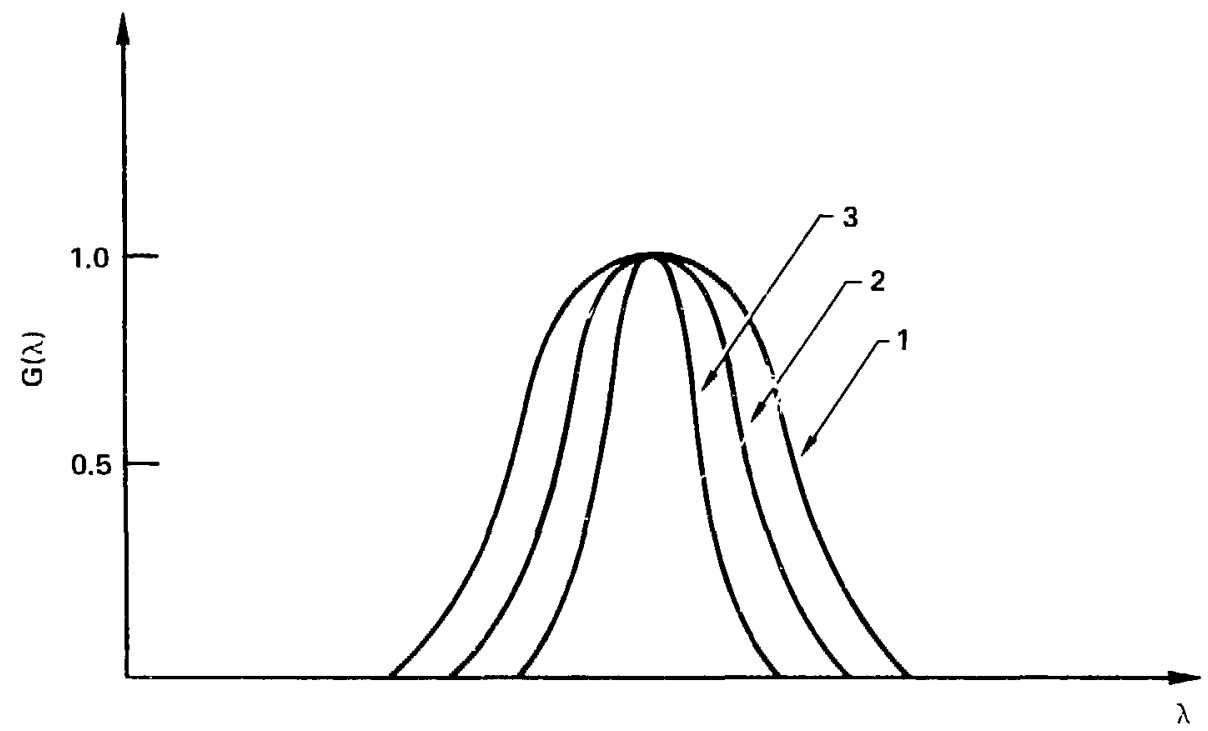

FIG. 21. Effective line narrowing caused by exponential gain. Line shape 3 is gain narrowed compared to initial line shape 1 .

\section{FOCUSABILITY}

The increase in radiance between the oscillator and the focusing lenses. duc to coherent gain through the amplifier, only occurs if the optical train does not degrade the phase front and reduce the power/sr. Such deterioration oceurs when the gain medium (gas or solid) or the beam-handling optics distort the beam phase front. An example of an interferogram from an optic producing such a deterioration is shown in Fig. 22. Aberrations such as these cause parts of the laser beam to diverge from the main-beam direction, causing interference ripple in the near field and "lobes" of energy to move away from the focal spot in the far field.

A simple but instructive way of looking at aberrations is that if a wave is differentially retarded in phase by $\mathrm{n} \lambda$ ( $\mathrm{n}$ waves) over an aperture $\mathrm{D}$, its focal position will be displaced by a distance ( $\mathrm{n} / \mathrm{D}$ ) . If only part of the wave front is unevenly retarded over a shorler distance $d$, the phase gradient is larger and energy will be "thrown" farther from the canter of the focal spot (see Fig. 23).

A more rigorous way of looking at focusing ${ }^{36.37}$ is to consider the lens as a transformation element. Because lenses can be made almost theoretically perfect from $f / 100$ to $5 / 0.4$, it is the laser optical quality, not the lens quality; that determines the amplitude and phase distrihution of the focused beam as it converges toward the focal plane. The lens transforms (with $t_{\text {lens }}$ ) the incoming beam by imposing a phase envelope (usually spherically converging ${ }^{6.38}$ ) onto the phase of initial beam.

$$
E^{\prime}(x, y)=t_{\text {lens }}\left(x, y^{\prime}\right) \cdot E(x, y)
$$




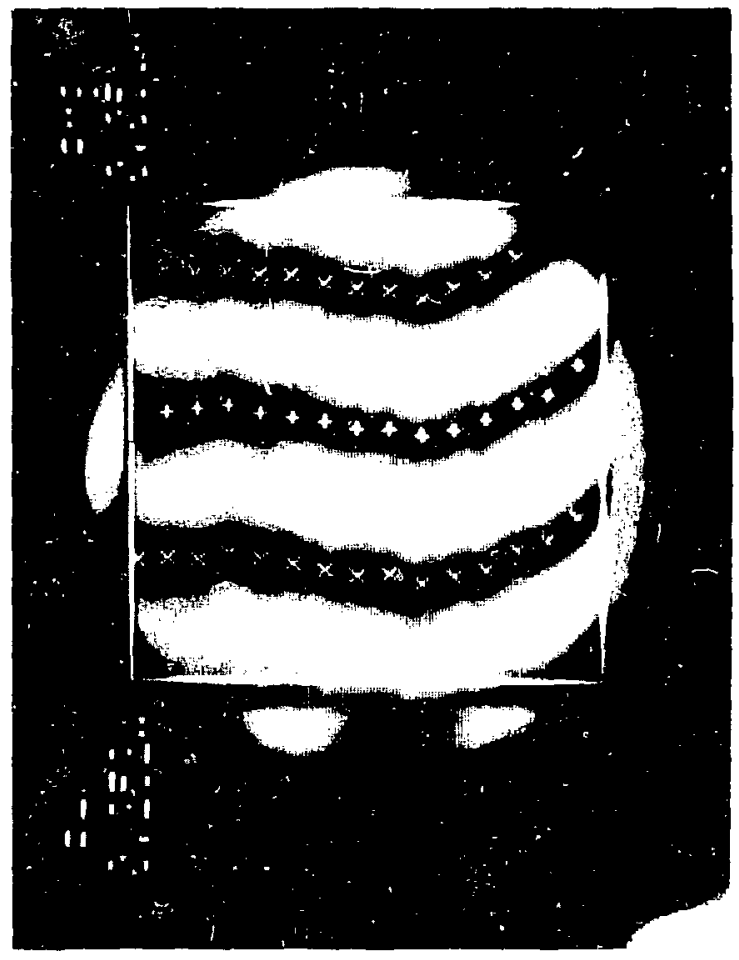

FIG. 22. Interferogram of a lens producing degraded phase front. The curvature of the lines shows thai about $\lambda / 2$ of phase aberration over the aperture will be imposed on a laser beam by this optical element.

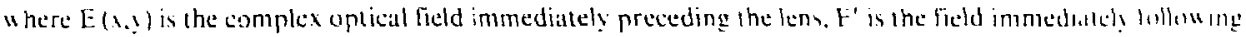
the lens, and $t_{\mathrm{len}}(x, y)$ is the lent transformation function. Fur paratial rats

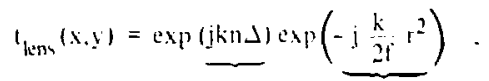

(1) (2)

where $\mathrm{k}$ is the wave number $2 \pi / \mathrm{A}$. $n$ the index of refraction. $\rightarrow$ the lens thickness. i the lens focial length, and $\mathrm{r}$ the distance from the beam axis to the measuring point. Term (1) is simply an overall phatse delay of $n]$, and term (2) shows that there is phase curvature $\mathrm{k}\left(\mathrm{r}^{2} / 2 \mathrm{f}\right)$. Visually, a picture of focusing is obtained as shown in Fig. 24. As the beam proceeds toward the focal point, the phase inlormation is converted to amplitude intensity and, when the beam reaches the focal plane, the phase information and amplitude information are displayed such that the intensity distribution vs $(x . y)$ at the focal plane is the power spectrum of the beam al the lens aperture: that is, the lens Fourier iransforms the beam. ${ }^{36}$

The Fourier-transform view of focusing yields some interesting elfects. e.g., a spatiai Gaussian beam envelope focuses to a Gaussian distribution at the focus, since the Fourier transform of a Gaussian is a (iaussian (see Ref. 16 for a complete description of Gaussian laser-beam focusing). A narrow (positive or negative) intensity spike. such as a $\delta$ function or the shadow behind a dirt spot. focuses over a very broad area (see fig. 25). This observation leads to the concept of spatial filtering 

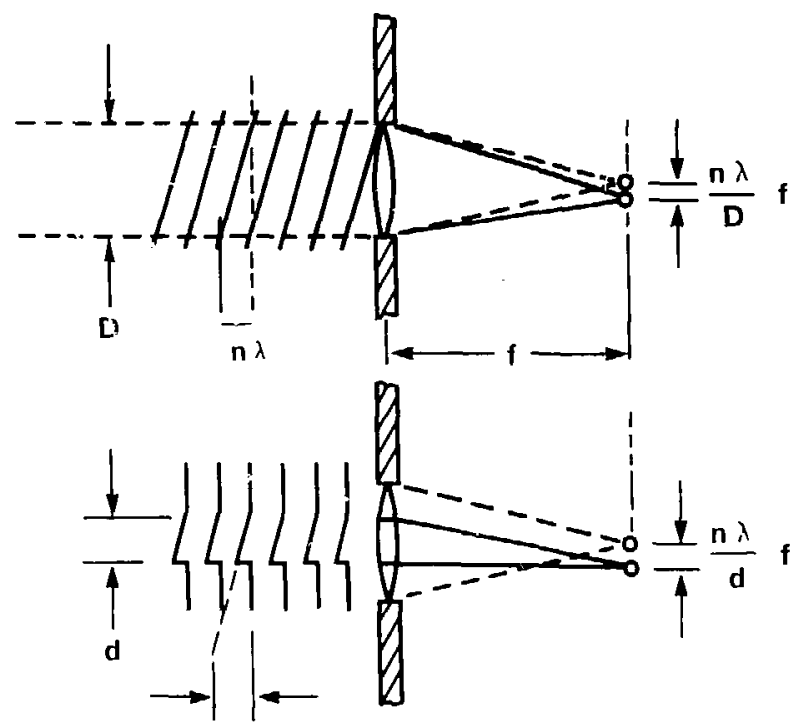

n $\lambda$

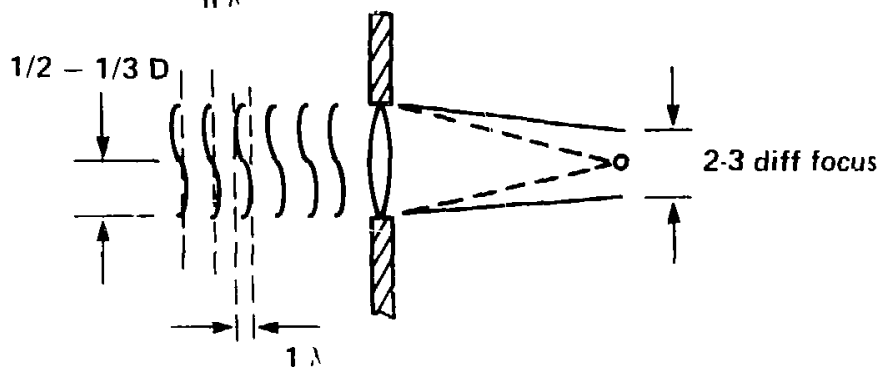

FIC:. 23. Phase aherrations steer the focul spot of the laser heam. Notice that it is the beam area affected by the gradient that determines the degree of focal deterioration.

A /ero-phase aberration beam, apertured by the lens holder, focuses 10 a spol that is deseribed by the Fuurer iransform of a round disk or the Airy pallern (e.g., see Fig. 26). ${ }^{34}$

The diameter $d_{f}$ of the focal spot to the first eero for a circularly apertured plane wave, with diameter $D$ and focul length $r$ is:

$$
U_{i}=2+\frac{\lambda}{\mathrm{D}} r \text {. }
$$

This is the hest focal spot possible for a circular heam of uniform phase and amplitude. For example. at $\lambda=1 \mu \mathrm{m}$ and $\mathrm{f} D=5$, the spot sige $\simeq 12 \mu \mathrm{m}$. For usual fusion-laser systems, depending on the laser Wavelength and the lens $\mathrm{C} / \mathrm{D}$ ( $\mathrm{f}$ number), focal spots are oblitined from $\mathrm{I} \mu \mathrm{m}<$ focus-diameter $-100 \mu \mathrm{m}$. If aberrations are present (Fig. 27) the focal spot is enlarged in proportion to the degree of the aberration.

In the introduction it was stated that the focal properties of most fusion lasers were sufficiently good that targets were usually irradiated in the converging heam. In this section the beam distribution at best focus was discussed because it is the limit to irradiation intensily and most importantly it is a useful diagnostic for detecting laser optical aberrations. The actual laser intensity distribution in the intermediate converging regime, between the laser-output beam profile and the hest-focus profile. is determined experimentally or with simulation codes. ${ }^{+11}$ 


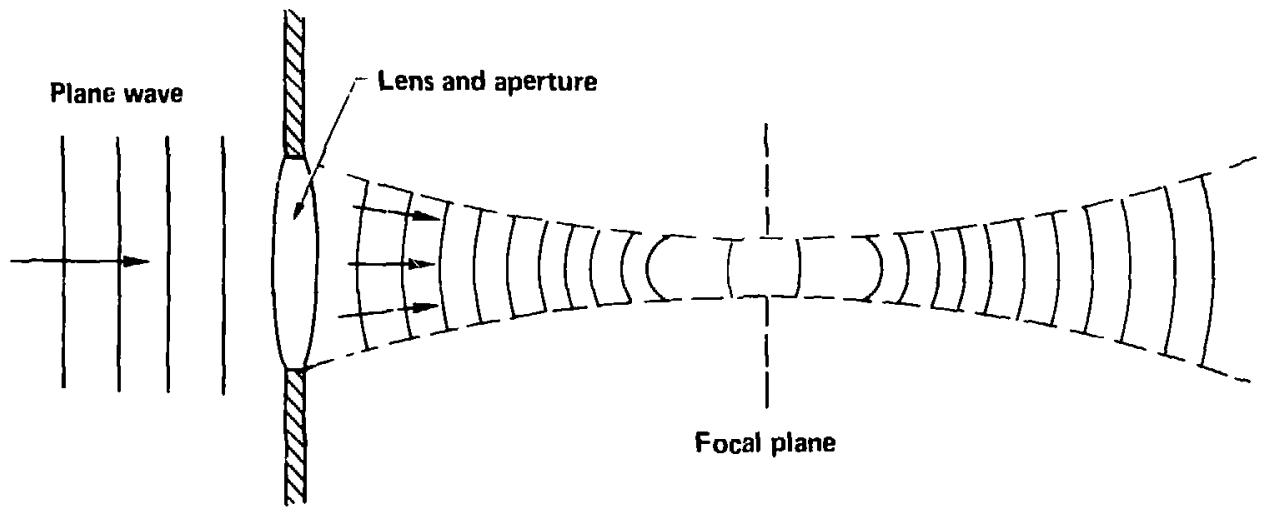

FIG. 24. Phase fronts are transformed by the lens to the Fourier power spectrum at the focus. 

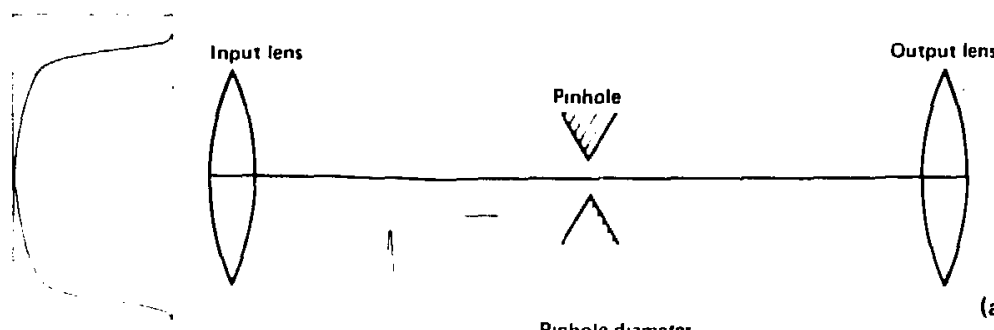

Pinhole diameter

(a)

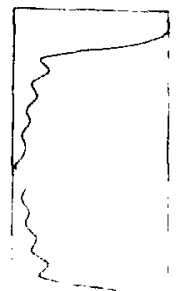

15 too small

Correct pinhole diamoter
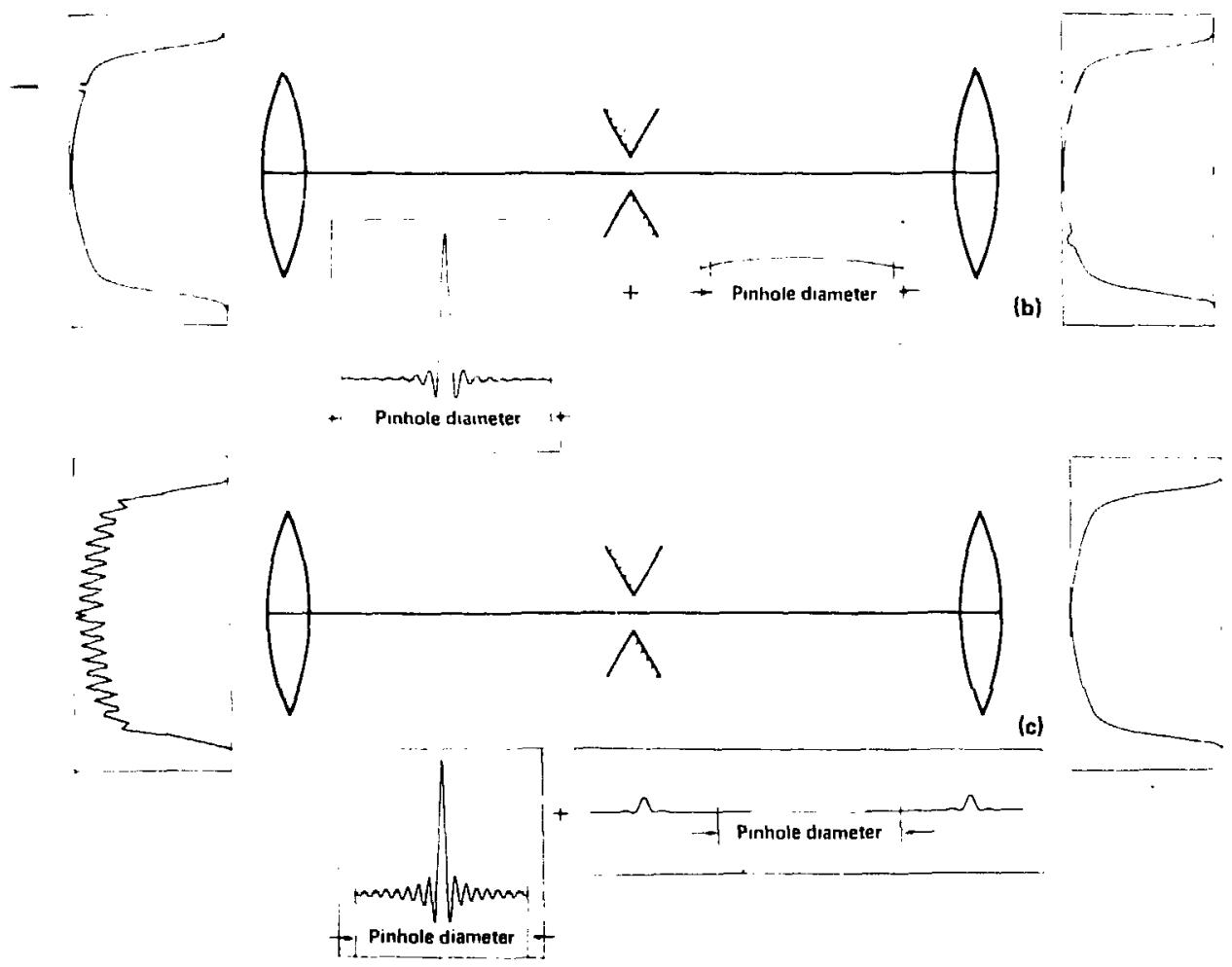

FIG. 25. (a) Pinhole truncating the focal pattern (Fourier transform) of incoming beam pattern at a position that leads to a rippled reconstructed beam. (b) Intensity spike is focused broadly at the pinhole while the beamenvelope transform passes through the pinhole. (c) Periodic ripple on a beam focuses outside the pinhole aperture, allowing a smooth beam profile to be reconstructed. Note that the pinhole is $\sim 10 \times$ larger than the diffraction limit of the main beam. 


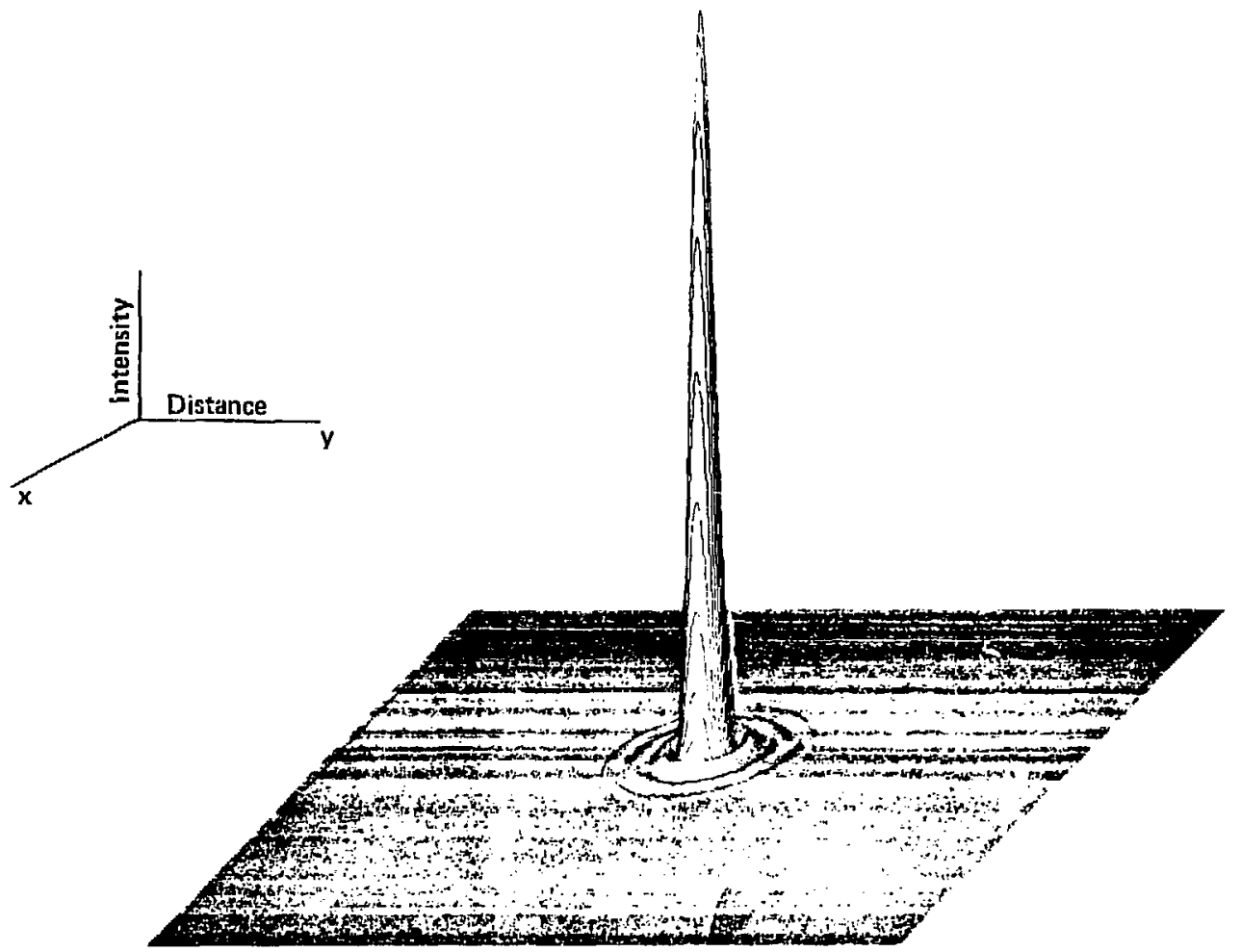

FIG. 26. Intensity distribution function for aberration-free circular pupil. 


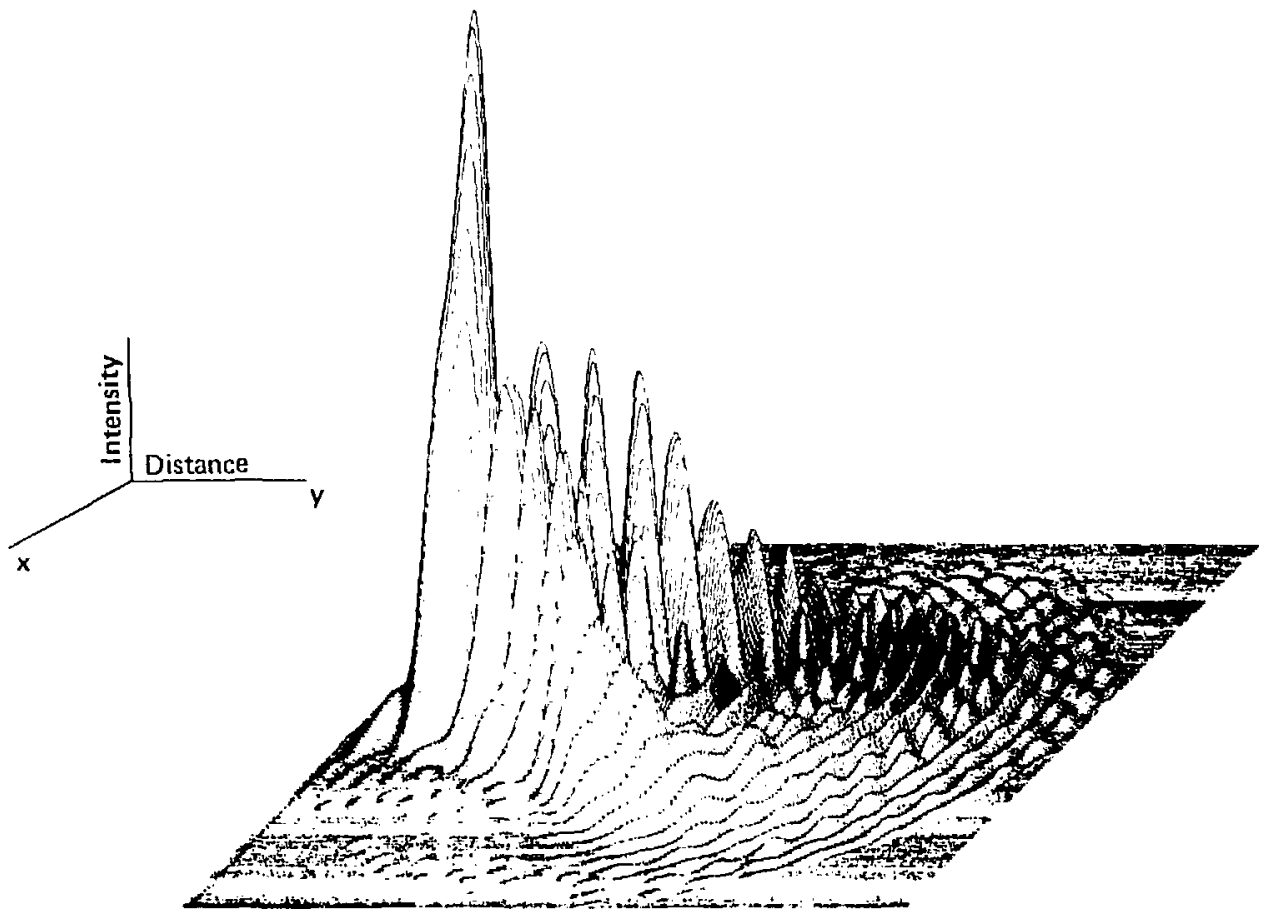

FIG. 27. Intensity distribution function for five wavelengths of third-order coma. 


\section{LASER OPERATIONAL CONSTRAINTS}

An oscillator and gain elements must be assembled so as to obtain a controllable, non-selfdestructing laser for experimentation. In this section the limitations imposed on gain media, by considerations of parasitic oscillation, amplified spontaneous emission, and chain self-oscillation. will be described. The additional limitations on the propagation of intense beams of light through laser components will also be described. These limitations arise because of linear losses, dielectric breakdown, self-frossing instabilities. intensity-dependent losses, and coherent intensification.

\section{GAIN CONSTRAINTS}

Amplifier gain is required to bring a pulse from the oscillator $(\sim 0.1-1 \mathrm{~mJ})$ to the desired full amplifier output $\sim 10^{3}-10^{4} \mathrm{~J}$. This requires a gain of $\sim 10^{6}$. Additional small-signal gain is reguired to overcome fixed losses and saturation. A single amplifier possessing a gain of $10^{6}$ would not be stable. If there were a reflectivity of only $10^{-6}$ (an unobtainably small value), this amplifier would break into uncontrolled internal oscillation, since the laser gain condition GR $>$ I would be satisfied. The division of the total gain into controllable sections (amplifiers) is clearly necessary (Fig. 28). An isolation element such as a Pockels cell or Faraday rolator is placed between each amplifier segment.

\section{CHAIN STABILITY}

For an amplifier section to be absolutely stabie, noise generated in the gain element must not grow to undesirable levels. $R_{1} R_{2} G^{2}$ must be less than 1 . Values for typical reflectivities are given in Table 4 .

An example of an oscillating laser element is shown in Fig. 29. Very high gain at the edge of a nonuniformly pumped rod causes oscillation to occur between the edges of metal apertures.

Conditional stability can sometimes be achieved by having the gain $G$ turned an in at short time period. as compared to the oscillation growth tinte associated with distance $f$ and reflectivities $R_{1}$ and $R_{2}$.

The intensity in the stage in Fig. 28 will grow from spontaneously emitted photons or from noise leaking through from a previous stage according to:

$$
\begin{aligned}
1 & =I_{0}\left(G^{2} R_{1} R_{2}\right)^{1 /(2(1 /))} \\
& =1_{11} \exp \left[\therefore \frac{1}{20} \ln \left(G^{2} R_{1} R_{2}\right)\right]:
\end{aligned}
$$

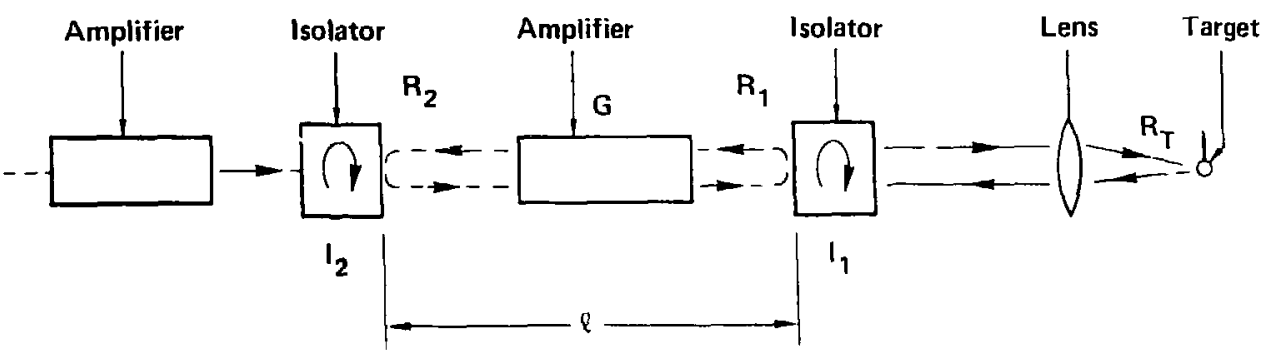

FIG. 28. Isolation of laser chain by isolators $I_{1}$ and $I_{2}$ having reflectivities of $R_{1}$ and $R_{2}$, respectively (neglecting reflections from other surfaces in the chain). Linear distance between two isolation elements is $t$, and $G$ is the gain at the time when the stability condition is examined. 
TABLE 4. Typical reflectivities of optical elements.

\begin{tabular}{ll}
\hline \multicolumn{1}{c}{ Optical element } & Reflectivity \\
\hline Uncoated glass-plate surface & 0.04 \\
AR-coated glass plate & 0.001 \\
Metal edge & 0.5 \\
Uniformly scattering damage or dust speck, & $0.001^{3}$ \\
\hline mm diam, backreflecting into $10^{-3} \mathrm{sr}$ & \\
\hline
\end{tabular}

${ }^{\mathrm{a} O}$ er its obscuration area neglecting diffraction.

thus the e-folding time is

$$
\tau_{\mathrm{U}}=\ldots \stackrel{2 \mathrm{Q} / \mathrm{C}}{\ln \left(\mathrm{G}^{2} \mathrm{R}_{1} \mathrm{R}_{2}\right)}
$$

Example: $G=20, R_{1}=0.01, R_{2}=1.0$ (in Fig. 28 assume that $I_{1}$ is absent and the target reflects $100 \%$ ), and $\ell=10 \mathrm{~m}$. Thus, $\tau_{\mathrm{e}}=4.8 \times 10^{-8} \mathrm{~s}$ and $10 \mathrm{e}$-folding times (for a gain of $2.2 \times 10^{4}$ ) occur in $480 \mathrm{~ns}$.

Very rupid gain switching must occur for this technique to be useful for isolation. This conditional stabilization is useful for amplifiers that can be pumped very rapidly ( $\sim 400 \mathrm{~ns})$, such as KrF, and for amplitiers that are located a long distance from the target, for example the Antares $\mathrm{CO}_{2}$ laser system at Los Alamos. For Nd:glass (pump $\sim 0.5 \mathrm{~ms}$ ) this technique is not useful as an isolation method.
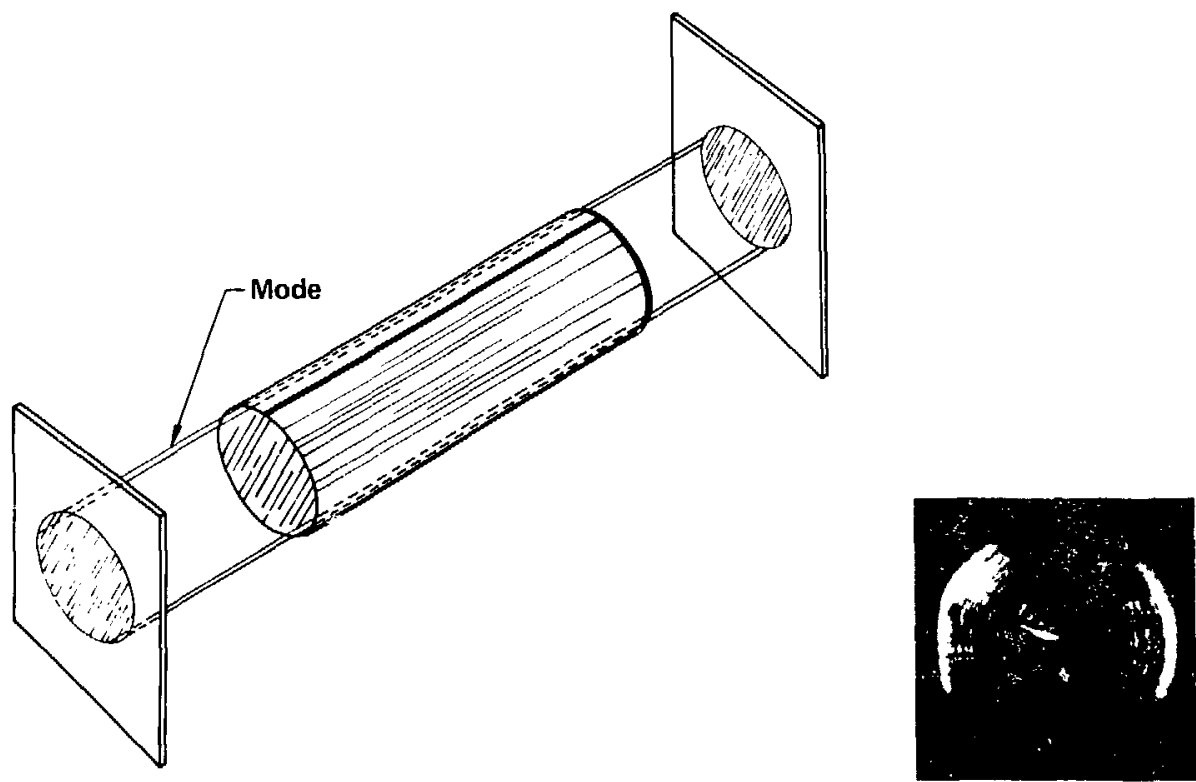

Parasitic oscillation at edge of $\alpha-\operatorname{rod}(\mathbf{G}>200)$

FIG. 29. Parasitic modes oscillating near edge of laser rod, where the nonuniform gain in this rod amplifier is highest. 


\section{TARGET ISOLATION}

Isolation from target reflection is required for both low- and high-power conditions. At very low power, a target placed exactly at best focus can serve as an almost perfect retroreflector, $\mathrm{R}_{\mathrm{T}} \sim 100 \%$ (Fig. 28). At high power targets typically reflect $10-20 \%$ of the incident radiation directly back in to the acceptance angle of the laser, and up to $70 \%$ back reflections have been observed under special target conditions. In addition, it is undesirable to place a passive isolator, such as a Faraday rotator, between the last gain element and the target ( $I_{1}$ in Fig. 28), because of its losses and damage problems. This situation imposes a more stringent constraini on the output gain section. The condition $R_{2} R_{T} G^{2}<1$ must be satisfied, where $R_{T} \sim 1.0$ and $R_{2}$ is constrained by realistic reflectivities (see Table 4). For $G \sim 10, R_{T} \sim 50-100 \%$, $R_{2}$ must be $\sim 1 \%$, a value that requires some attention to achieve. A parasitic mode can easily develop in an amplifier chain (especially a chain with no spatial filter restricting the mode propagation angles) by scattering from the target at nne end and the high reflectivity of a metal lens holder, screw head, or even a damage spot on a coating. The oscilation can rapidly develop to a level that destroys the target.

High-power isolation from target retroreflection will be required in the laser-output gain section because eventually $I_{r u f} G_{r}>I_{\text {damage. }}$ In this case the gain for the reflected pulse $G_{r}$ will usually be lower than the small-signal gain because the forward propagating pulse has reduced the laser inversion level. By proper design an isolator at $\mathrm{I}_{2}$ in Fig. 28 will suffice, or one can use a fast-closing pinhole isolator, such as a plasma device, ${ }^{4-43}$ or, in special circumstances, add an output isolator stage $l_{1}$ and accept the limitations of its additional nonlinear phase contributions and lower damage threshold,

An example of isolation, the Shiva laser isolator scheme, ${ }^{44}$ is shown in Fig. 30 . In this example the propagation is traced in the backward direction for both polarizations. This is required because of the polarization sensitivity of disk amplifiers and Faraday rota'ors. The Faraday rotators are noted as FR and the Pockels cells as PC.

\section{TRANSVERSE OSCILLATIONS}

In the previous sections, the stability requirements for isolating amplifiers from each other and from the target were discussed. Similar considerations oceur within the individual amplifier. ${ }^{45-47}$ The stored gain within the amplifier is such that if internal reflectivity is not controlled, the amplifier will break into osciliation internally and the gain level will be clamped at the oscillator threshold condition inside the disk, $G^{2} R_{1} R_{2}<1$. Examples of modes are shown in Fig. 31 and gain clamping is shown in Fig. 32.

Figure 32 shows examples of the onset of parasitic modes that "clamp" the gain at an undesirably low level in a laser-disk amplifier. ${ }^{+8}$ In the two lower curves the edge reflectivity of the laser disks was not low enough to prevent the onset of internal oscillations.

\section{AMPLIFIED SPONTANEOUS EMISSION (ASE)}

In most laser systems, the amplifiers are excited beforz the laser pulse passes through and is am. plified. During this "quiescent" lime, spontancous emission from the carly amplifier stages can be amplified to levels that may damage the laser target. The amplificd spontaneous cmiscion develops along with the amplifier gain. In Nd:glass systems ${ }^{40}$ it occurs over the 600- $\mu \mathrm{s}$ flashlamp pump pulse, in $\mathrm{CO}_{2}$ over the $-5 \mu \mathrm{s}$ sustainer pulse, and in $\mathrm{KrF}$ over the E-beam pulse period of $\sim 100-500 \mathrm{~ns}$.

Figure 33 is an example of fluorescence emission from a volume of Nd:laser material being amplified by subsequent amplifier stages. The geometry is similar to that shown in Figs. 5 and 7.

The energy reaching the target is $13 \mathrm{~W} / \mathrm{cm}^{2} / \mathrm{sr} \times 1 / 4 \pi(5 \mathrm{~cm})^{2} \times 10^{-8} \mathrm{sr} \times 10^{-3} \mathrm{~s} \times 10^{6}$ gain $=2.6 \mathrm{~m} J$. (Spectral line narrowing describud earlier redures this value by about four.)

This energy may be sufficient to damage fragile targets being used for experiments. The emission can be controlled by breaking up the amplifier gain path with fast-opening isolators such as Pockels cells or rotating shutters. Note that spatial filters reduce the ASE reaching the target. 


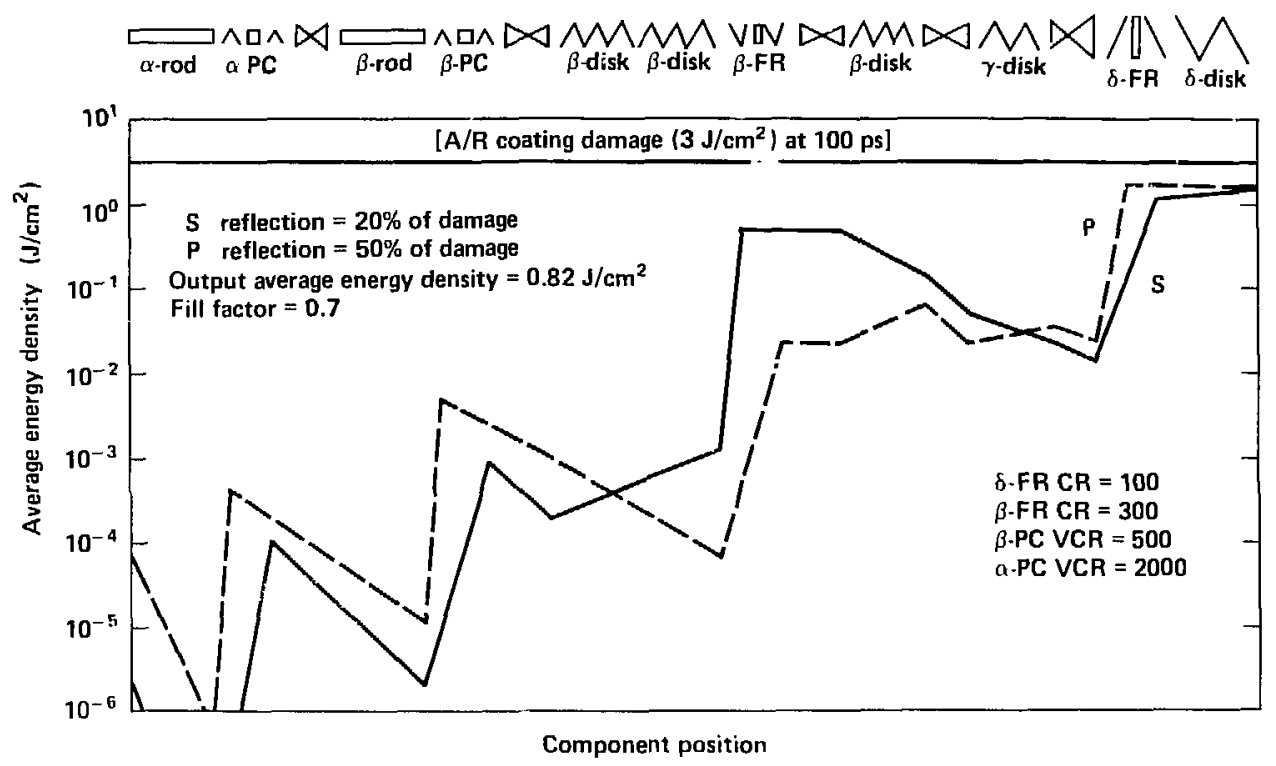

FIC. 30. Isolation of Shiva laser with gains and damage thresholds appropriate for 100 ps pulse propagation. vote how the $S$ reflection (cross polarization) gruws in intensity as it propagates backward from the $\gamma$ disti to the if disk. ( $R$ and $Y C R$ arc isolation ratios.

Parasitic mode within amplifier box

Total internal reflection mode in laser disk
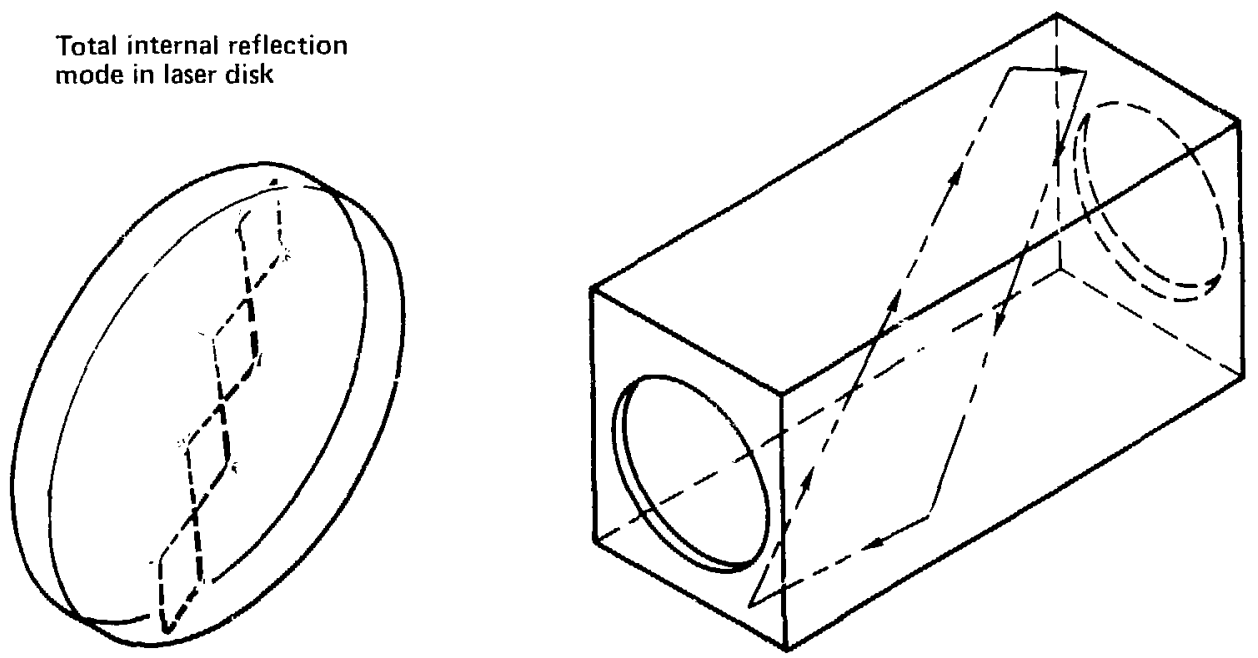

FIG. 31. Internal parasitic modes within laser amplifier structure. 


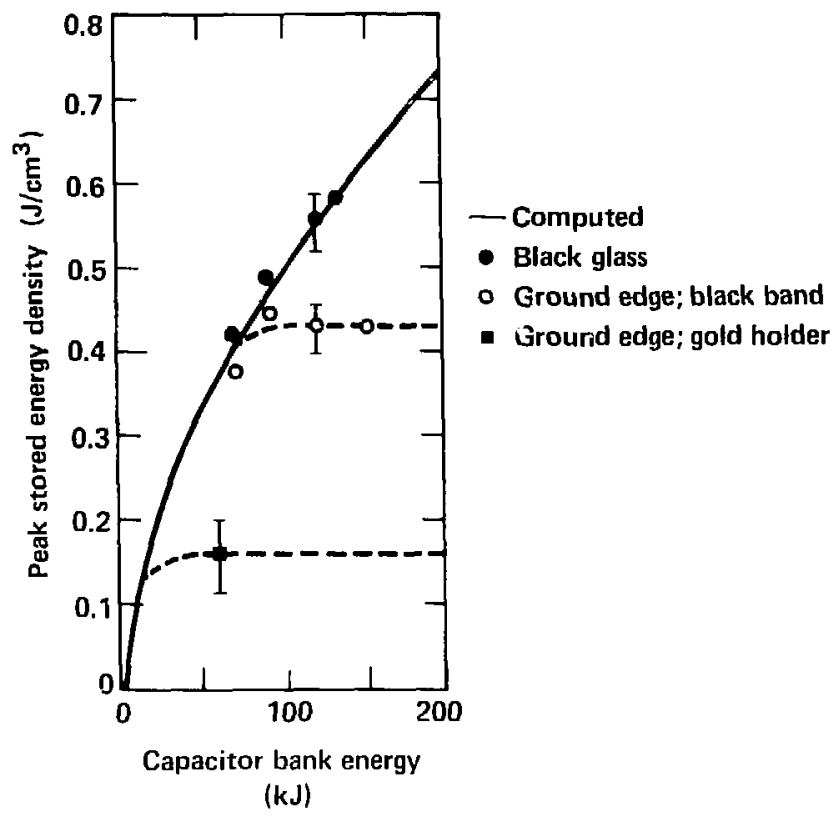

FIG. 32. Stored energy (gain) in 6.4-cm-diam disk laser a mplifier as a function of flashlamp ene 'gy and disk edge preparation. Vote the very low gains that occur when the edge reflectivities are high.

Figure 34 shows the ASI signal measured from a Shita Vaglass laser chain. ${ }^{90}$ In Shiva, the small-

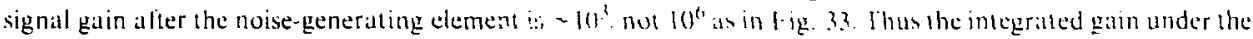
curve following the hashlamp pumping is acceptatly low. However. when the carly stage isolation Pochels cells open, additional noise from an earlier part of the chain shows up as a short intense norise pulse.

amplified spontaneous emission alse oceurs within an indiudual laser amplificr and appears als at loss of stored inversion. When the gain coefficient-length product approathes five a $1 .+5$. the loss al inversion calused by amplified spontaneous emission exceds the natural huorescence loss of the latser medium. ${ }^{4}$ As a result. the excitation of high-gain amplifier media must oceur at a rate significintly more rapid than the rate determined by the fluorescence time scale. For example. Vd:glass laser disks are most efficiently flashlamp pumped on the time scale of the fluorescence (spontaneous emission): thus amplified fluorescence is an important de-excitation mechanism.

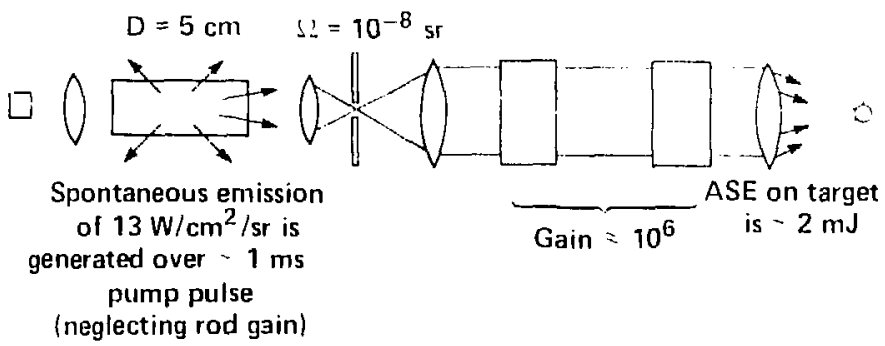

FIG. 33. Amplified spontaneous emission generated in early chain stages. 


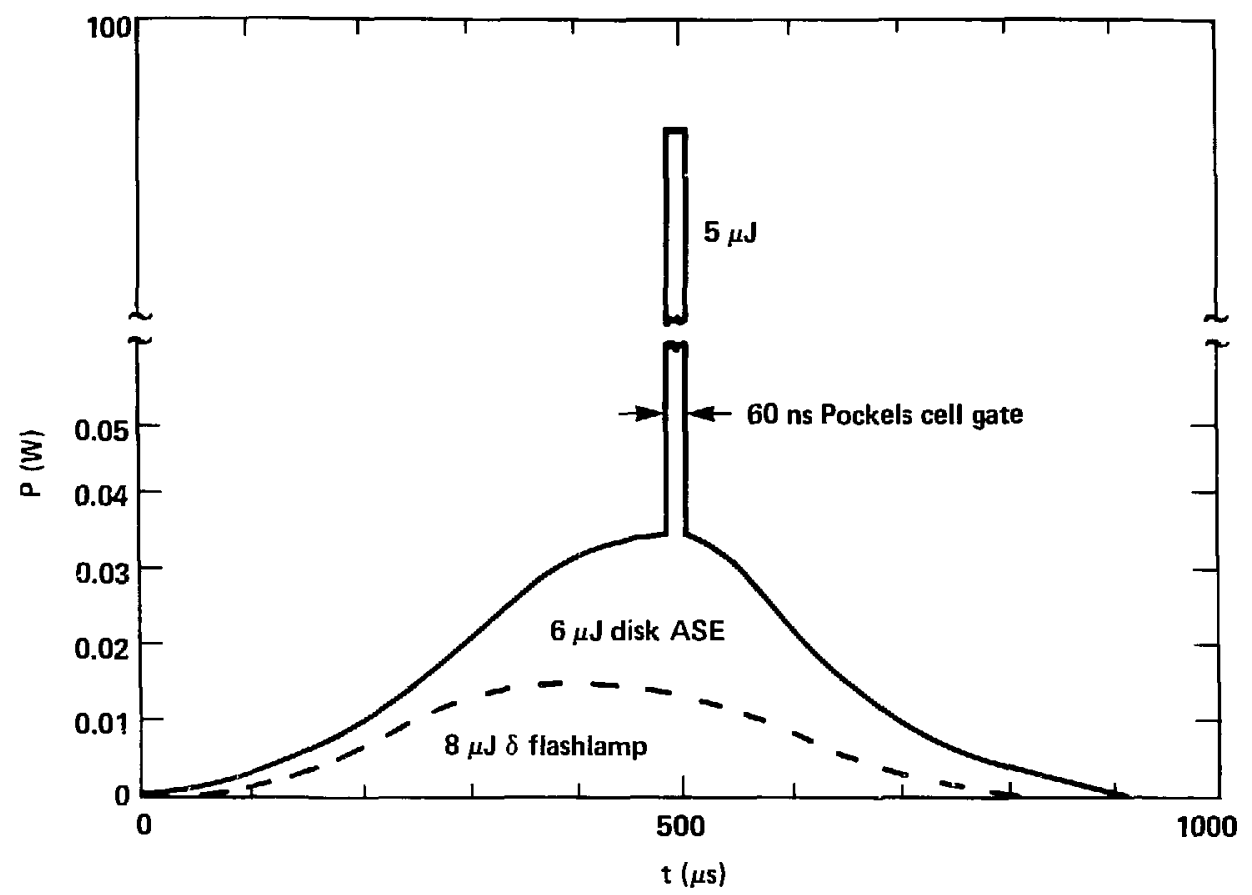

FIG. 34. Shiva amplified spontaneous emission (ASE) on target from a single arm. Measured with $30 \mathrm{TW}-100$ ps laser settings.

\section{LOSSES}

As the laser beam travels down the amplifier chain, it experiences net gain and increases in intensity. However, the net gain is the difference between the laser medium gain and the inevitable losses. These losses arise from several sources. The major losses occur from Fresnel reflection from dielectric interfaces and passive losses in amplifier media or optical components. Loss of beam energy is undesirable because it is costly to recoup. In a 10-kJ laser beam, a $0.1 \%$ loss costs $10 \mathrm{~J}$ per occurrence and a $7 \%$ Fresnel loss costs $700 \mathrm{~J}$, a loss greater than many small research lasers produce. Loss is especially severe in a saturated gain situation, where the beam gains energy slowly by extracting energy in proportion to the linear passage through the media. but loses energy at loss elements in proportion to the tolal beam fluence. Table 5 lists some linear losses in optical materials.

Additional low-level losses occur in laser systems, and these losses are not easily quantified. They are usually associated with clipping of the beam on an aperture, with dirty optics, with losses at the spatial-filter pinholes, etc. Gain reserve is required in a laser to overcome these losses.

\section{NONLINEAR OPTICS}

At power densities of $10^{9}-10^{10} \mathrm{~W} / \mathrm{cm}^{2}$, nonlinear optical processes begin to occur as the laser beam passes through the system optics. Coherent and sequential multiphoton effects occur because the laser dielectric media respond nonlinearly to the very intense laser beam. These phenomena are both detrimental and 
TABLE 5. Linear losses in some optical materials.

\begin{tabular}{|c|c|}
\hline Material & Linear loss \\
\hline Laser glass & $\begin{array}{l}-0.002 \mathrm{~cm}^{-1} \text { bulk } \operatorname{loss}_{5} 21,51-53 \\
<0.001 \text { surface loss } 58,59\end{array}$ \\
\hline Optical glass BK-7 & $\begin{array}{l}0.002 \mathrm{~cm}^{-1} \text { bulk } \operatorname{loss}^{54-56} \\
<0.001 \text { surface loss } 58,59\end{array}$ \\
\hline Faraday rotator glass & $0.005 \mathrm{~cm}^{-1} 57$ \\
\hline AR coating & $0.001 /$ surface $^{60}$ \\
\hline $100 \%$ turning mirror & $\begin{array}{l}0.001 / \text { surface }^{60} \\
(-57 \text { Ioss if mirror is partially } \\
\text { transparen f for diagnostics) }\end{array}$ \\
\hline $\begin{array}{l}\text { Antive } \mathrm{CO}_{2} \text { laser } \\
\text { ges neixture }\end{array}$ & $0.0017 \mathrm{~cm}^{-161}$ \\
\hline NaCJ lenses & $0.001 \mathrm{~cm}^{-1} 61,62$ \\
\hline AR coating on $\mathrm{NaCl}$ & $0.001 /$ surrace 60 \\
\hline Germanium polarizer & $0.015 \mathrm{~cm}^{-1} 61,62$ \\
\hline
\end{tabular}

TABLE 6. Nonlinear phenomena in laser materials at $10^{9} \mathrm{~W} / \mathrm{cm}^{2}$.

\begin{tabular}{|c|c|c|}
\hline Phenomenon & $\lambda(0.25 \mu \mathrm{m})$ & $\lambda(1 \mu \mathrm{m})$ \\
\hline $\begin{array}{l}\text { Two-photon } \\
\text { absorption } 63,64\end{array}$ & $\begin{array}{l}0.05 \mathrm{~cm}^{-1} \text { loss } \\
\text { coefficient in } \\
\text { lused silica. }\end{array}$ & $\begin{array}{l}<0.01 \mathrm{~cm}^{-1} \text { in } \\
\text { laser glass } \\
0.001 \mathrm{~cm}^{-1} \\
\text { in silica }\end{array}$ \\
\hline $\begin{array}{l}\text { Self-focusing and } \\
\text { beam breakup } 65-70\end{array}$ & $\begin{array}{l}\mathrm{e}^{28} \text { growth/m in } \\
\text { fused silica }\end{array}$ & $\begin{array}{l}\mathrm{e}^{3.4} \text { growth/m in } \\
\text { silicate laser glass }\end{array}$ \\
\hline $\begin{array}{l}\text { Whole-beam phase } \\
\text { retardation } 71-73\end{array}$ & $\begin{array}{l}2.27 \mathrm{~N} / \mathrm{m} \text { path } \\
\text { length of fused silica }\end{array}$ & $\begin{array}{l}0.27 \mathrm{~N} / \mathrm{m} \text { of silicate } \\
\text { laser glass }\end{array}$ \\
\hline $\begin{array}{l}\text { Stimulated Raman } \\
\text { conversion } 74 \text { (vibrational) }\end{array}$ & - & $\begin{array}{l}0.01 \mathrm{~cm}^{-1} \text { gain } \\
\text { coefficient in } \mathrm{N}_{2} \\
\text { at } 1 \mathrm{~atm}\end{array}$ \\
\hline $\begin{array}{l}\text { Stimulated rotational } \\
\operatorname{Raman} 7 \bar{j}\end{array}$ & - & $\begin{array}{l}2 \mathrm{~cm}^{-1} \text { gain coefri- } \\
\text { cient in } \mathrm{H}_{2} \text { at } 1 \mathrm{~atm}\end{array}$ \\
\hline $2 w$ harmonic conversion 76 & - & $\begin{array}{l}250 \% \text { encrgy conver- } \\
\text { sion in } 1 \mathrm{~cm} \mathrm{KDP}\end{array}$ \\
\hline
\end{tabular}

useful. A list of processes relevant to high-power laser operations is shown in Table 6 . While most of these phenomena have been extensively studied with small $(\sim 0.1-\mathrm{cm}$ diam) laser beams interacting over $\sim 1-\mathrm{cm}$ interaction dimensions, the high-power laser designer must be concerned with large-scale manifestations of these phenomena over $\geqslant 10-\mathrm{cm}$ beam diam and $>100-\mathrm{cm}$ interaction lengths.

These phenomena are listed first because of their crucial importance in determining the propagation limits in laser design, and second bec use of their potential usefuiness in increasing the flexibility of presently available systems.

\section{SELF-FOCUSING}

Small-scale sel[-focusing is the major propagation limit for short-pulse laser systems. ${ }^{77}$ Self-focusing occurs because the index of refraction of the dielectric optical material is equal to a field-independent term $n_{0}$ plus a field-dependent term $n_{2} E^{2}$. 
TABLE 7. Nonli.ıear phase retardation.

\begin{tabular}{lcc}
\multicolumn{1}{c}{ Material } & $\begin{array}{c}\gamma\left(\mathrm{m}^{2} / \mathrm{W} \text { at }\right. \\
1.06 \mu \mathrm{m})\end{array}$ & $\begin{array}{c}\gamma\left(\mathrm{m}^{2} / \mathrm{W} \text { estimated }\right. \\
\text { at } 0.25 \mu \mathrm{m})\end{array}$ \\
\hline $\begin{array}{l}\text { Silicate Iaser glass (ED-2) } \\
\begin{array}{l}\text { Fluorophosphate laser glass } \\
\text { (E-181) }\end{array}\end{array}$ & $3.8 \times 10^{-20}$ & - \\
$\begin{array}{l}\text { Optical glass BK-7 } \\
\text { Fused silica }\end{array}$ & $1.5 \times 10^{-20}$ & - \\
\hline
\end{tabular}

Thus

$$
n=n_{0}+n_{2}\left\langle E^{\mathbf{2}}\right\rangle
$$

or, rewritten in terms of the beam intensity,

$$
n=n_{0}+\gamma l
$$

Typical values ${ }^{21,73}$ for $\gamma$ are given in Table 7 .

At an intensity of $10^{10} \mathrm{~W} / \mathrm{cm}^{2}$, the term $\gamma 1$ for silicate laser glass ${ }^{53}$ is $3.8 \times 10^{-6}$. A beam at this propagation intensity passing through $\mathrm{I} \mathrm{m}$ of this glass will experience a phase retardation of $3.6 \times 10^{-6} \mathrm{~m}$, of 3.6 waves at $1 \mu \mathrm{m}$. A uniform phase retardation of this nature is not particularly serious, although it does lead to time-dependent beam aberrations. ${ }^{72.78}$ However, this effect leads to a serious propagation instability known as "small-sciale" self-focusing. Growth of small-scale low-level beam ripples occurs because the nonlinear interaction causes power to transfer from the main-beam mode to the smaller-scale ripple modes via a parametric interaction. ${ }^{65}$ Figure 35 schematically demonstrates the effect. Figure 36 shows the results of an experiment ${ }^{68}$ showing this effect.

In a real luser system the major sources of the low-level ripples are diffraction ripples caused by dust particles, damage centers, clipped apertures, or interference fringes from incompletely coated optics or spatial filtering. The fastest-growing ripples $(\sim 0.5-1 \mathrm{~mm}$ wide) will grow in relative intensity (when viewed at the focus of a lens where both phase and amplitude aberrations appear) as given by ${ }^{79}$ :

$$
N=N_{0} \exp (2 B)
$$
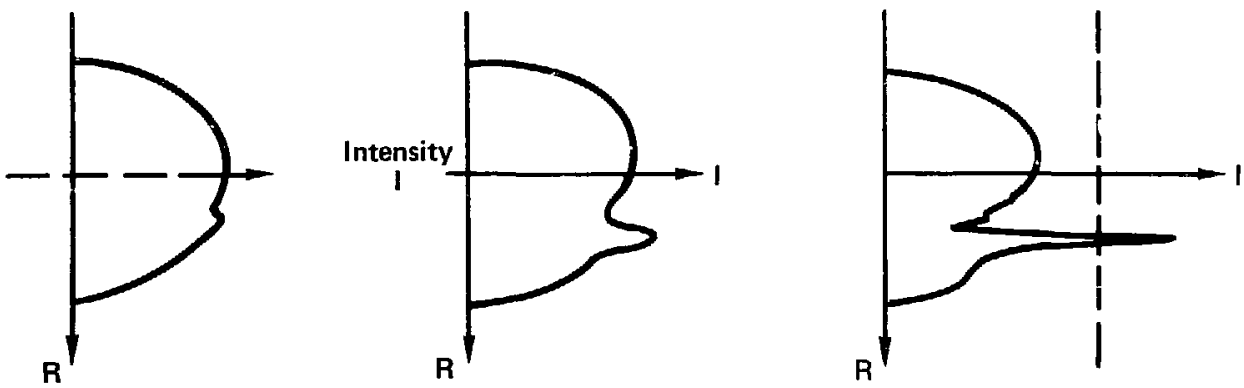

Damage threshold

FIG. 35. A small-amplitude (or phase) nerturbation can grow in intensity at the expense of the main beam untensity until damaging levels are reached or until the total beam has filamented and become unfocusable. 
where

$$
B=z_{\text {val }}^{2 \pi} \int_{0}^{x} \gamma l d z
$$

and

$$
\begin{aligned}
& N=\text { noise amplitude relative to } \mathrm{N}_{0} . \\
& \mathrm{N}_{0}=\text { initial noise amplitude, } \\
& \lambda_{\text {vac }}=\text { laser wavelength in vacuum, } \\
& \gamma \quad=\text { nonlinear phase retardation coefficient }\left(\mathrm{m}^{2} / \mathrm{W}\right) \\
& 1 \quad=\text { beam intensity in the optical material, and } \\
& \gamma \quad=\text { length of opticial material through which beam travels. }
\end{aligned}
$$

Experimentally 11 hats heen found that values of $B=3-5$ (depending on cleanliness and relay uplics) are a propagation limit. ${ }^{15,77}$ Thus, . $^{2 B} \simeq 400$ and, if ripple grow th does not exceed 100 ; beam anplitude (i.e., when $\mathrm{N}=1$ ), the initial noise $\mathrm{N}_{0}$ must he 1400 or 0.0025 of the main-heam envelope amplitude. It is also important to note that $B$ is simply the beam-phase retardation in radians? 2 B $=3.14$ is equivalent 10.1 Inonlinear phase relardation.

The intensification caused by self-locusing instability is of great concern to the laser designer and must be controlled. Lack of control reduces focusability by converting the high-radianci main-beam envelope

$$
\begin{aligned}
& \text { Spatial treq }=2 / \mathrm{mm} \\
& I_{\text {peak }}=5 \mathrm{GW} / \mathrm{cm}^{2} \\
& \text { Gain }=7 \% \mathrm{~cm}^{-1}
\end{aligned}
$$

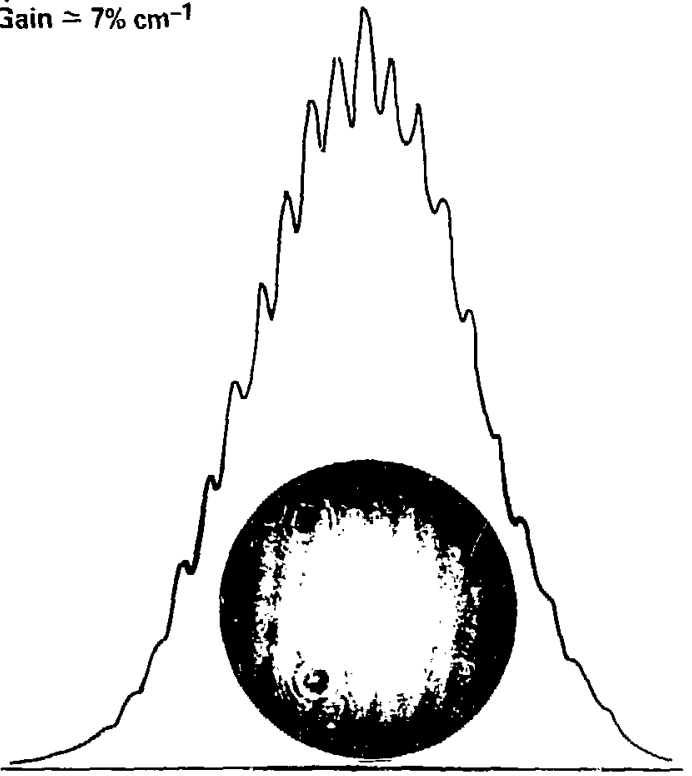

Input

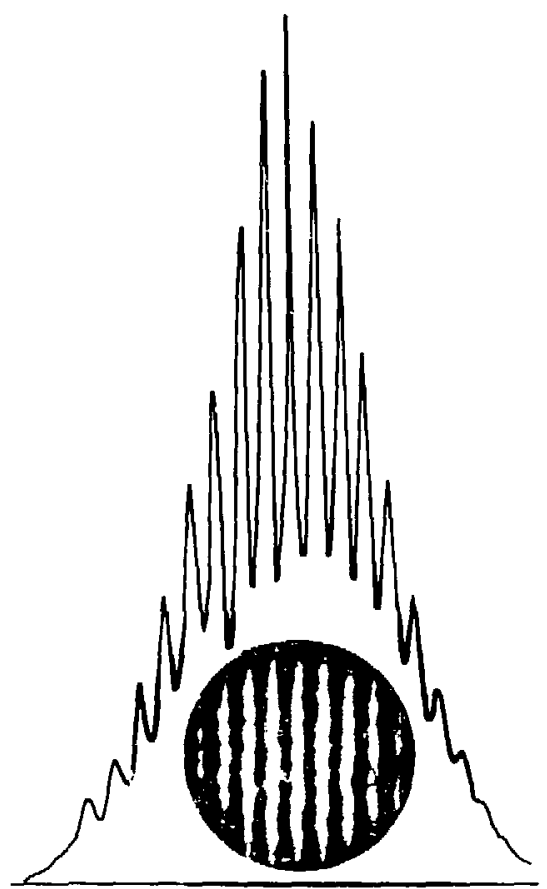

Output after propagating $24 \mathrm{~cm}$

FIG. 36. A purposefully modulated beam was propagated through a 24-cm rod of amplifier glass. The near-field ripple intensity was seen to grow exponentially. 


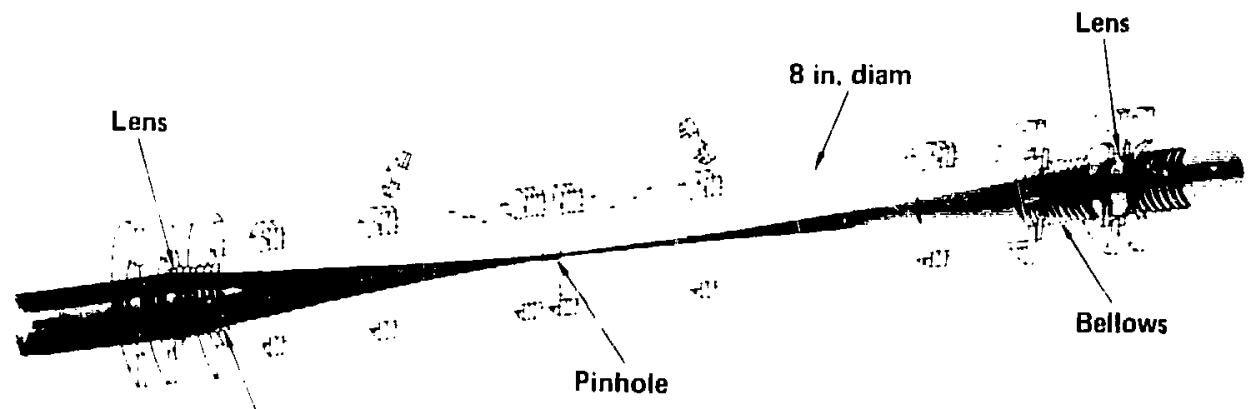

Bellows
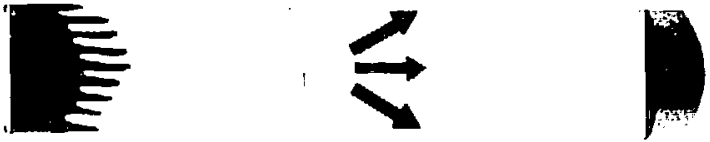

FI(i. 37. High spatial frequencies have divergent focusing angles which do not pass through the pinhole.

into line nonfucusable filaments and cituses laser damage where the inlense filaments exced damage thresholds. In order to maintain B $? 4$, the laser chain must be divided into subsections where the ripple intensilication can he reduced by spatial filtering ${ }^{8(1.8)}$ (see figs. 37 and 38 ). That is. the nuise level $N$ must be reduced to a manageable level $V_{0}$ whenever $B-+t$ in the laser system. High-frequenty spatial noise is reduced by spatial filtering with a lens and pinhole combination. An example of this techniq!ae applied to a 9 -em laser beam is shown 1.ig. 38 .

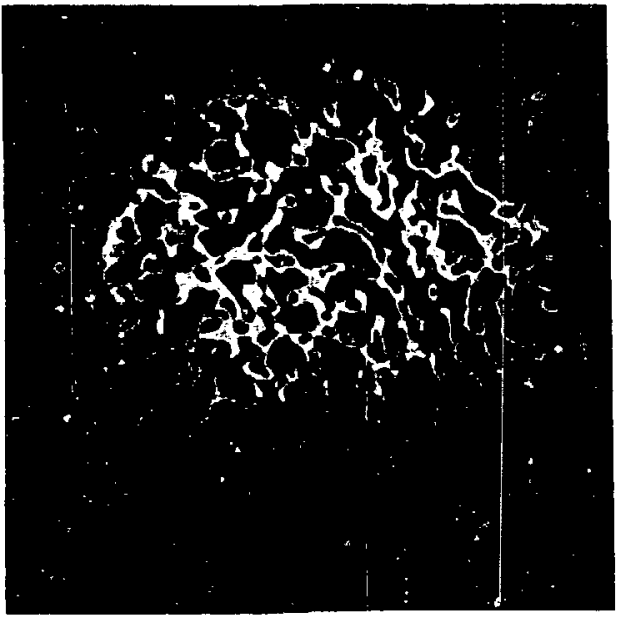

Into spatial filter

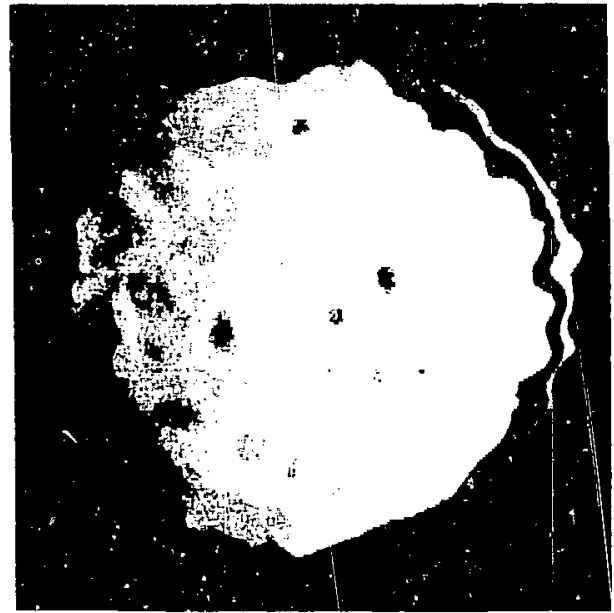

Exit from spatial filter

FIC; 38. Reduction of high-frequency spatial noise by a lens and pinhole combination as applied to a 9-cm laser beam. 
In system design the laser parameters in Eq. (14), such as beam intensity, glass path length, and material $\gamma$ are manipulated. This manipulation is to minimize system cost while maximizing performance, and wil! be discussed in the last section of this report. The goal of the laser designer is to attain maximum performance from eath laser-chain stage within the constraint of $B \leqslant 4$ between spatial filter segments, and a total nonlinear phase-retardation constraint of $\Sigma B_{i}-10-12$.

\section{DAMAGE LIMITS}

The ultimate determination of a laser system's output aperture is the beam intensity that can be transmitted through a given aperture. Thus, a $10 \mathrm{~kJ}$ laser built with a $1 \mathrm{~J} / \mathrm{cm}^{2}$ damage constraint requires $10^{4} \mathrm{~cm}^{2}$ of output aperture $\left(113 \mathrm{~cm}\right.$ diam), whereas at $10 \mathrm{~J} / \mathrm{cm}^{2}$ only $10^{3} \mathrm{~cm}^{2}(36 \mathrm{~cm}$ diam $)$ is required. Since the cost of a laser increases approximately in proportion to the total aperture area. the goal is to operate at as close to the maximum-permissible propagation values as possible. Material selection has a profound influence on laser design because variations of $2-10 X$ in propagation limits can occur.

The phenomena that limit the laser-fluence iniensity vary with laser wavelength, material, and beam intensity. In the long-pulse regime (where the long-pulse region is defined as not inducing nonlinear optical effects) the propagation limits are set by dielectric breakdown, which occurs most often on surfacess or on thinfilm coatings on surfaces ${ }^{82,83}$ (see Fig. 39).

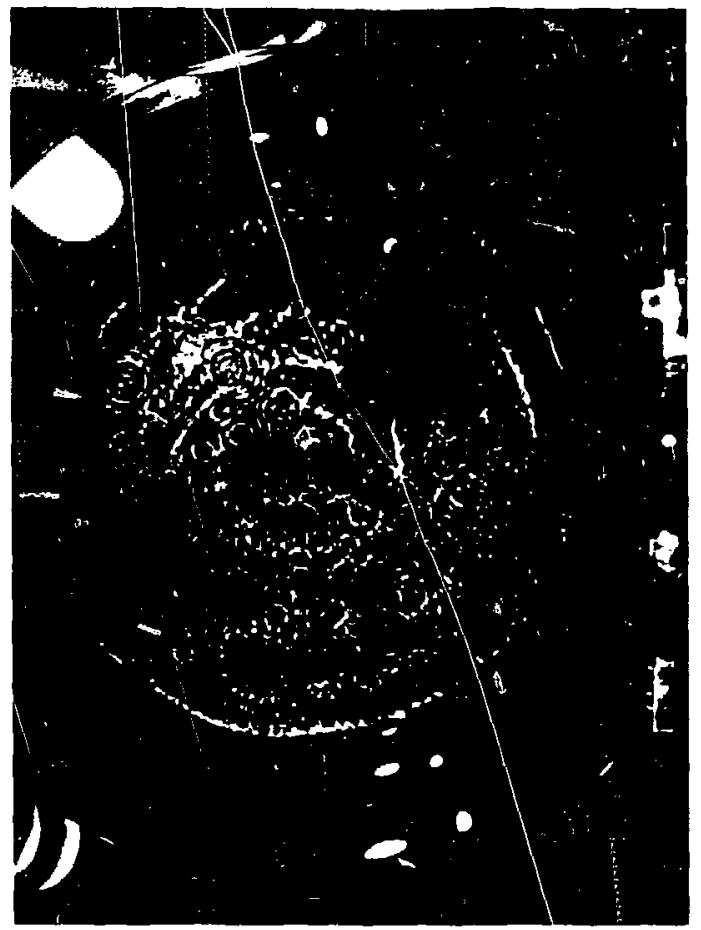

FIG. 39. Example of optical damage on an antireflection (AR) coating. 
In this regime damage mechanisms are primarily associated with the heating of absorbing impurities until local material destruction begins. Then massive absorption of beam energy occurs and the materal is permanently altered over an area larger than the initial breakdown site. The actual initiation of the material breakdown is the subject of a great deal of research and controversy. Figure 40 shows the progressive development of a damage site on a thin-film coating. ${ }^{54}$ The damage appears to begin at the interface layer between the substrate and the coating layers. as evidenced by the lifting of the outer layers in (b). The damage begins at a localized site and then spreads, resulting in a completely damaged optic as shown in (c). A great deal of effort hal heer expended to raise the thresholds for damage, with some 'uccess. ${ }^{85}$

Typical damage levels for both surfaces and material "bulk" are shown in Tables 8 and 9. Bulkmalerial damage thresholds are strongly affected by the presence or absence of inclusions. ${ }^{86}$ Absorbing incluvion in glass can lower the damage threshold to $<5 \mathrm{~J} / \mathrm{cm}^{2}$ : however, commercial laser-glass formulations by detinition are free from these problems.

While the lists in Tables 8 and 9 are incomplete. they should give the reader a feeling for the variability in coating-damage thresholds as a function of production method and wavelength regime. To design a complex multicomponent laser system, operating luence levels must be selected that are safely below commercially avaitable component-damage levels measured at the desired pulse duration of the laser system.

The ubove discussions centered on damage limits without nonlinear effects. In the short-pulse aperitung regine, the short-pulse propagation limit is imposed bo the accumulation of noniinear-beam aberations as diseussed previously. The result of the nonlinear aberrations is either to make the beam unfocusable or to raise local beam ripples to the damage level of the optical system, before the average fluence of the laser bean reaches a desirable level. In the case where the constraint is actual surface damage. the machanism : $:$ material breakdown with short pulses may be different from that for long pulses. 89.90

TABLE 8. Typical I-ns single-shot damage thresholds. ${ }^{87}$ The first value givent in a two-value number (e.g.. 5-15 J) is for production contings, the second is for research one-of-a-kind coatings.

\begin{tabular}{|c|c|c|c|}
\hline & $0.25 \mu \mathrm{m}^{\mathrm{a}, \mathrm{b}}$ & $1.0 \mu \mathrm{m}$ & $10 \mathrm{~mm}$ \\
\hline \multicolumn{4}{|l|}{ Optical surfice } \\
\hline Ouartz (quary fillm at $22 \mathrm{~ns}$ ) & 3.8 & $15-20$ & $\mathrm{~N}^{\mathrm{C}}$ \\
\hline $\mathrm{VaC7}(\mathrm{NaF}$ film nt $22 \mathrm{~ns})$ & 1,11 & is & 7 \\
\hline$B K .7$ & 3 & $15-20$ & $\mathrm{~N}^{\mathrm{C}}$ \\
\hline Antireflection coated surface & 1 & $5-12$ & 6 \\
\hline $100 \%$ reflector surfice & 1,3 & $8-12$ & 14 \\
\hline \multicolumn{4}{|l|}{ Bulk miterial damage } \\
\hline Ouartz & $\overline{N 5}$ & 40 & $\mathrm{~N}^{\mathrm{C}}$ \\
\hline BK-7 & $\mathrm{ne}^{\mathrm{N}}$ & 40 & $\underset{N C}{N}$ \\
\hline $\mathrm{NaCT}$ & - & - & 10 \\
\hline Gie & $\therefore$ & $\mathrm{N}^{\mathrm{C}}$ & $0.2^{\mathrm{h}}$ \\
\hline
\end{tabular}

${ }^{3}$ First value at $0.1-\mathrm{ns}$ pulse, second value at $22 \mathrm{~ns}$.

bransmission Ioss caused by plasma formation. actual damage not observed.

ENot transparent."

TABLE 9. Damage levels in $\mathrm{J} / \mathrm{cm}^{2}$ of materials as a function of pulse duration for $1 \mu \mathrm{m}$ laser light. ${ }^{88}$

\begin{tabular}{lcccc}
\hline & $0.1 \mathrm{~ns}$ & $1.0 \mathrm{~ns}$ & $3 \mathrm{~ns}$ & $20-30 \mathrm{~ns}$ \\
\hline Optical glass surface & 7 & 15 & 25 & $100 \mathrm{~J} / \mathrm{cm}^{2}$ \\
AR conating & 4 & 5 & 6 & $20-40$ \\
$100 \%$ reflectur & 6 & 8 & 10 & $16-50$ \\
Bulk glass fevel & - & 40 & - & 100 \\
\hline
\end{tabular}



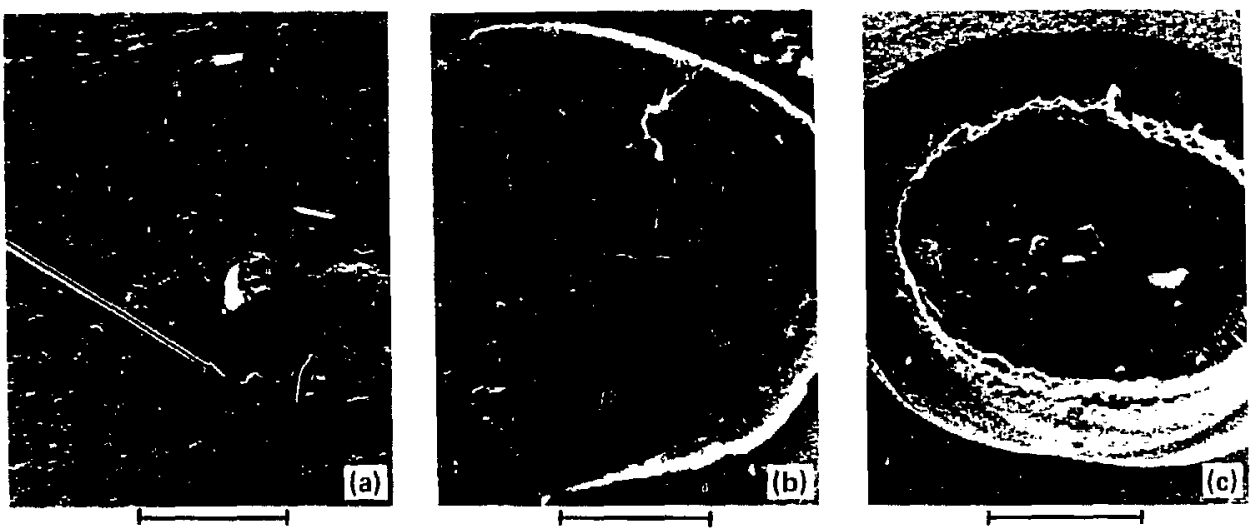

FIG. 40. Damage morphology of four-layer Shiva $\mathrm{AR}$ coating ( $\mathrm{TiO}_{2}$ against substrate, then $\mathrm{SiO}_{2}$, $\mathrm{TiO}_{2}$, and $\mathrm{SiO}_{2}$ ). The scale har is $1.0 \mu \mathrm{m}$ long. (a) The area indicated by the arrow shows incipient spall of surface-coating layers. (b) Surface layers lifting from substrate. (c) Thin-film layers removed and melted residue on substrate.

\section{LINEAR PHASE-ABERRATION CONSTRAINTS}

An additional constraint on propagation is the accumulation of linear phase aberrations. As mentioned earlier, with present fusion systems these have not yet been a serious limitation. However, as reactor laser designs are contemplated, ${ }^{91}$ the quality of the beam phase front is critical to the achievement of long stand-off distances and precise focusing. In gaseous lasers, the ultimate limitations to beam focal quality are the density fluctuations in the medium. $\lrcorner \rho / \rho .^{92}$ In solid lasers, the cumulative polishing errors and material homogeneity set the aberration level. In addition, fuctuations in the air path between the laser and the target can also cause severe beam aberrations. Terhniques to analyze cumulative aberrations for fusion applications are based on work by Vlasov et al. ${ }^{93}$ and are briefly discussed by $\mathrm{Haas}$ and Giass. ${ }^{94}$

\section{APERTURE SIZE, FILL FACTORS, AND INTENSIFICATION}

Several physical processes have been discussed that ultimately determine laser-system performance. One of the most important is the optical-component damage levels. The actual determination of permissible laser-operating fuence levels ultimately decides the laser output fluence. ${ }^{95}$ Thus. a great deal of effort hats been expended in maximizing the fluence-per-unit-area in a given laser system. In addition, one maximizes the average fluence through a given aperture by working to develop a smooth ripple-free beam. However, as has been seen, diffraction effects and small-scale self-focusing raise the peak beam fluence above the average beam fluence. A perfect system and a real system are shown in Fig. 41.

In Fig. 4l(b), the average fluence (or usage of the output aperture at the amplifier exit) is redaced because of the following arguments. The beam intensity at $r=R_{0}$ (aperture edge) must be equal to rero, otherwise diffraction ripples from the aperture edge will propagate into the heam center. This causes a reduction in $2 \pi \int f(r) r d r$ from $\pi R_{0}^{2} /$ max to $f \pi R_{0}^{2} /$ max, where $f$ is the fill factor, or fraction of fill compared to it "square" beam. The radial shaping of light beams to enhance their focal or propagation properties is called "apodization." By using relay imaging lechniques, little radial beam shaping is required compared to previous efforts. References 10 beam apodization efforts are given in Refs. 96-98. The best fill factor that can presently be obtained by using image relaying ${ }^{99}$ is $f \sim 0.8$. Even with perfect relaying, diffration from dirt, from inadvertent "edge clipping." or from self-focusing intensification, raises intensity peaks above the abcage mainbeam intensity. Since permissible fluence is determined by peak fluence, the average heam energy must be somewhat less than $\left[\pi R_{0}^{2} /\right.$ damuga. 


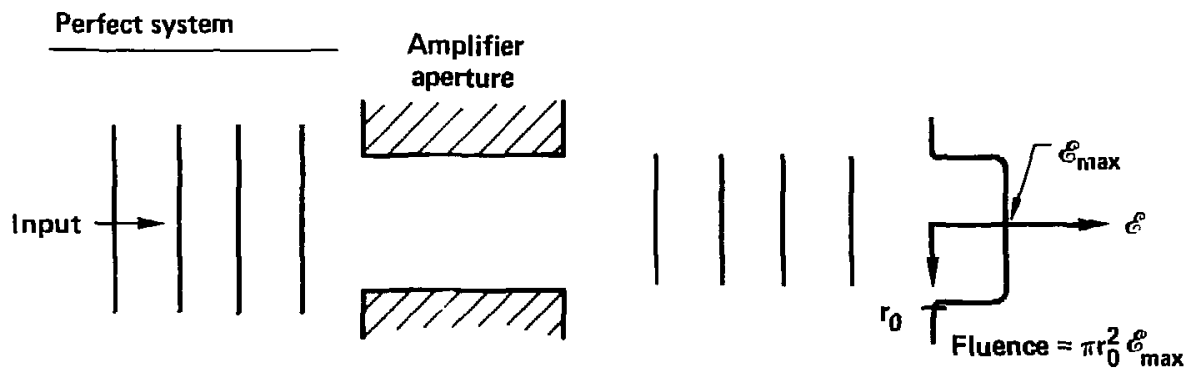

\section{Real system}
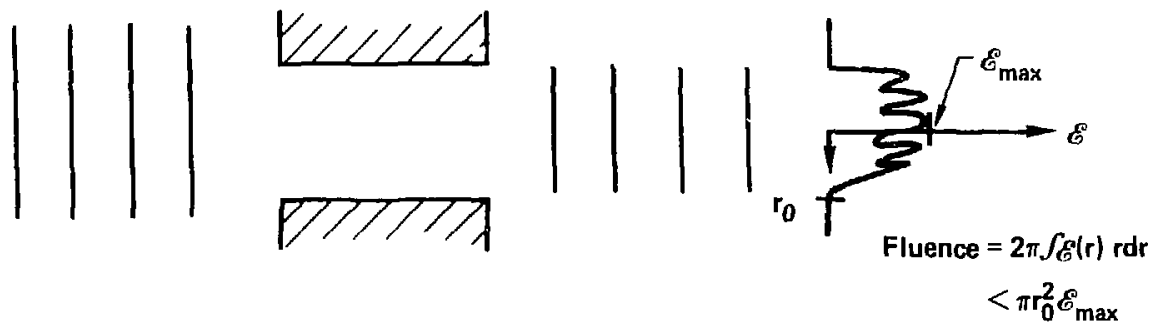

FIG. 41. Apertures limit beam shape and nonuniform beam amplitude vs radius reduces fill factor.

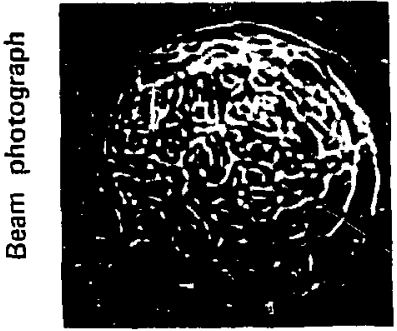

Dec. 1976

partially relayed

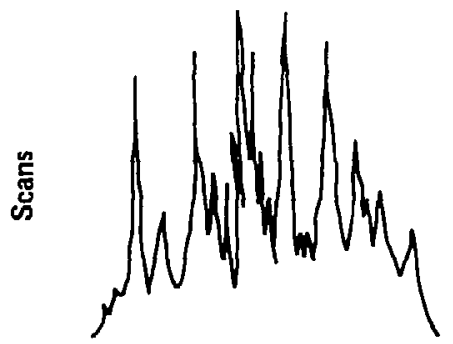

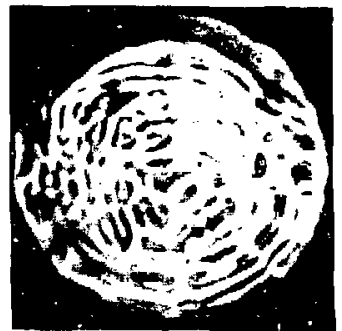

Dec. 1977

fully relayed

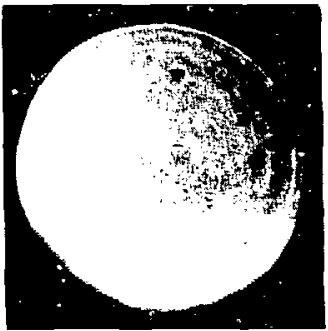

Dec. 1978 ultra "clean"

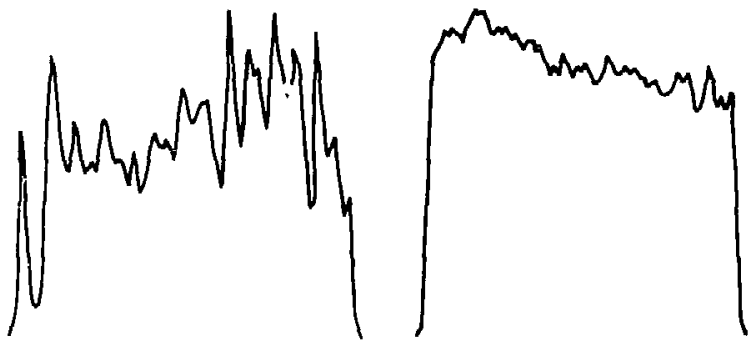

FIG. 42. Improvement in beam quality of Argus laser at the 2.5 TW per beam intensity level. 
Typical beam photographs taken on the Argus laser ${ }^{100}$ are stown in Fig. 42. They demonstrate the improvements that have occurred on this system as beam fill factor was improved by reducing noise sources and sharpening the edges. In Fig. 42 it is apparent that the peak-to-average fluence margin has been reduced from $>4$ to 1 down to 1.3 to 1 . However, if damage sites occur or beam misalignment vecurs with consequent "edge-clipping," >2-to-1 beam modulation would reappear and damage would thus oc nu if the system was designed to operate at 1.3-to-1 modulation levels. A system designed to operate at 1.3-to-1 modulation levels requires careful maintenance, but the performance benefit is very high because the aperture is used very effectively.

\section{COHERENT INTERFERENCE}

Sources of modulation other than those described previously are associated with coherent reflections from surfaces, particularly on lenses, although tilted polarizer plates, beam splitters or diagnostic plates, and windows can cause reflections that coherently interfere and lead to intense standing waves and opical damage. ${ }^{101}$ The source of these intense standing waves is the coherent addition of electric fields from forward and reflected or scattered waves.

If the iields at point $\mathrm{p}$ in Fig. 43 are coherently added (assuming only two interacting fields) large modulations occur when the reflected wave adds in-phase or out-of-phase.

$$
I(p) \simeq\left(E_{1} \pm E_{2}\right)^{2}=E_{1}^{2} \pm 2 E_{1} E_{2}+E_{2}^{2} \text {, assuming } E_{1} \gg E_{2} \simeq E_{1}^{2}\left[1 \pm 2\left(E_{2} / E_{1}\right)\right]
$$

Thus

$$
I(p)=I_{1}\left[1 \pm 2\left(1_{2} / 1_{1}\right)^{1 / 2}\right] \text {. }
$$

Example: If a secondary reflection $\mathrm{I}_{2} \simeq 0.01 \mathrm{I}_{1}\left(1 \%\right.$ of $\left.\mathrm{I}_{\text {inciden }}\right)$,

then

$$
\left(I_{2} / I_{1}\right)^{1 / 2} \simeq 0.1
$$

$$
I(p)=I_{1}(1 \pm 0.2) \text {, a } 20 \% \text { modulation. }
$$

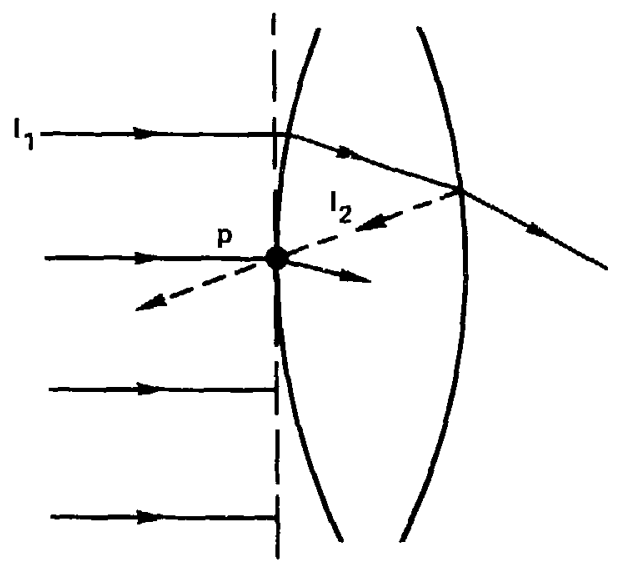

FIG. 43. At point $p$ a reflection from an uncoated surface is interfering with an incoming wave $I_{2}$. This drawing assumes no reflection from the front surface, otherwise the coherent interference of three waves must be considered. 


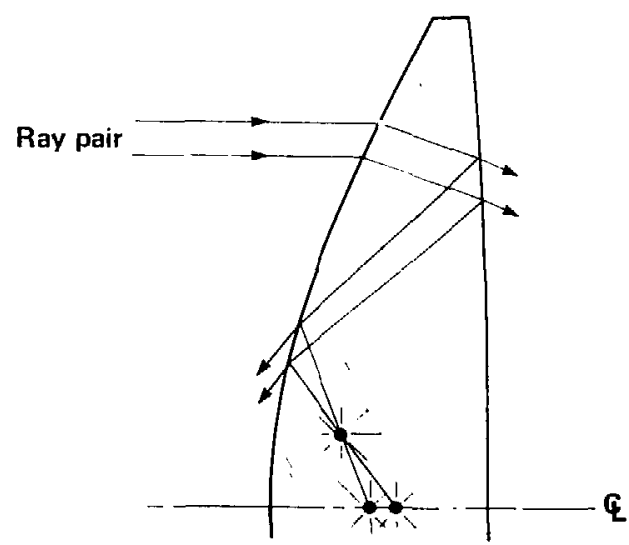

FIG. 44. Ghost-focus analysis. The incoming ray pair partially reflects from several surfaces until they cross at a "caustic" where the intensity can be great enough to cause damage.
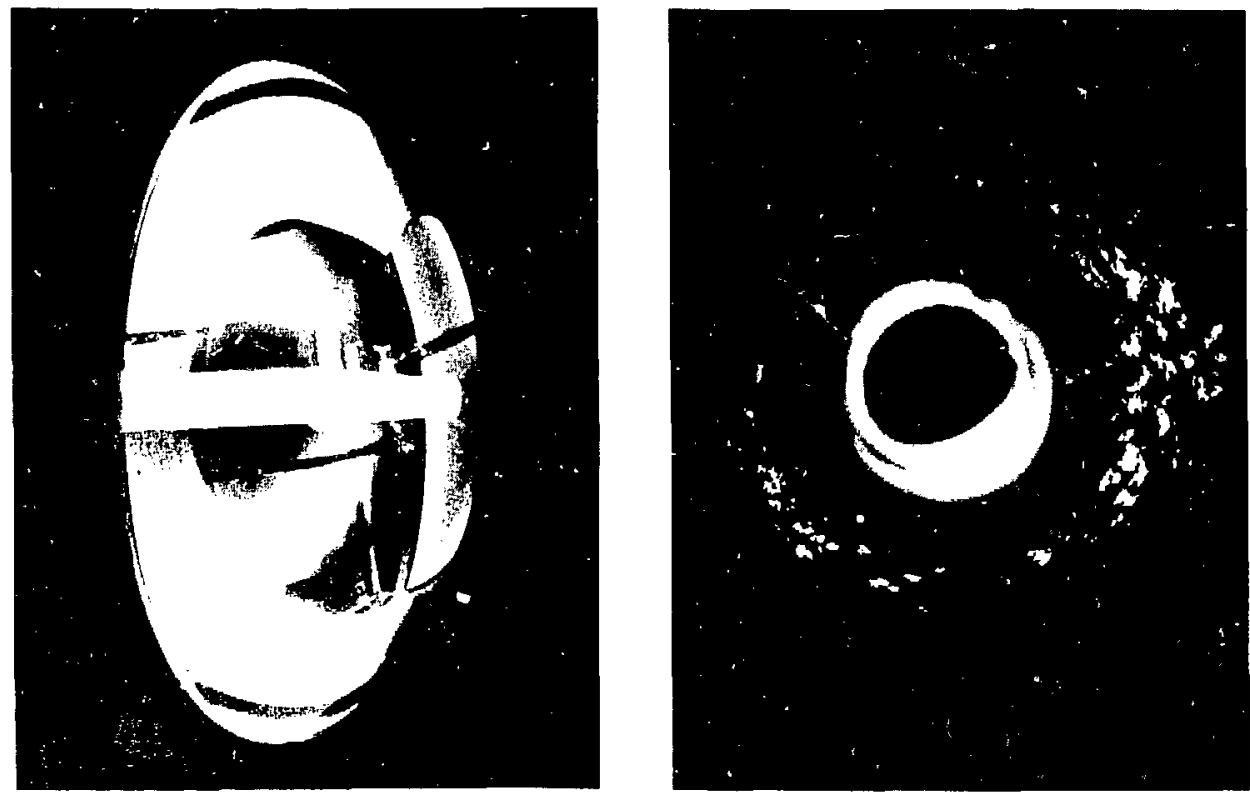

FIG. 45. Iens on left ( $f / 1)$ shows ghost-focus damage caused by off-axis caustics near center hole. Image on right is expanded view of damage around hole. Proper lens design is important to avoid problems for both forwardpropagating laser beam and backward-propagating reflected beam from target. 
Such modulation often occurs in diagnostic beam photographs if care is not taken to $A R$ coat all beamsplittet surfaces. Similar effects can occur in optics within the laser chain.

In addition to the coherent effects, secondary reflections from laser components also cun focus tc cause both on-axis and off-axis "caustics," leading to system damage. These are called "ghost" foci and car occur in the lens or in another part of the laser system where the focal point occurs. 102 Figure 44 shows: sketch of the intensification process. On-axis caustics inevitably occur, but are eliminuted by boring a hule ir the lens as shown in Fig. 45.

\section{LASER COMPONENTS}

Now consider the components that are assembled to make up a laser system. In the previous sections, the physical constraints that limit components have heen presented. These included small-signis gain. stored energy, isolation, damage, etc. In this section component issues pertinant to fusion lasers are presented. The components functions included here are pulse generation (oscillation), amplification, spatial filters and relays, and isolators.

\section{OSCILLATORS}

The generation of the desired laser pulse shape for injection into a power amplifier chain is the subject of oscillator design. As intense a pulse as possible is desired, consisteni with damige limits. 11 musi possess the desired pulse shape in time. the desired bandwidth, and the desired spatial shape. In particular. a timebandwidth-limited, Gaussian-in-time, single-transverse spatial-mode pulse is us'dally desired for subsequent spatial and temporal pulse shaping and amplification (Fig. 46).

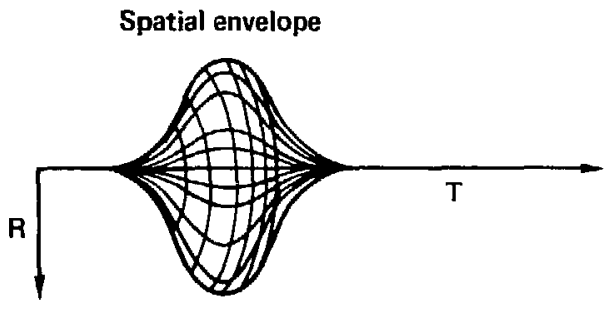

Optical substructure

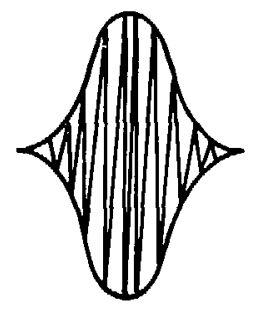

FIG. 46. Gaussian spatial and temporal pulse. 
The literature of oscillator design will not be reviewed here other than to present some of the basic ideas relevant to fusion lasers.

In an earlier section, an example illustrated the development of laser radiance as the initial Mluorescence noise passed through a sufficiently long amplifier medium. In this way, the very low radiance associated with spontaneous emissions of $\approx 10 \mathrm{~W} / \mathrm{cm}^{2} / \mathrm{sr}$ was amplified to the $>10^{17} \mathrm{~W} / \mathrm{cm}^{2} / \mathrm{sr}$ required for target irradiation. However, unless the frequency and spatial development of the electromagnetic wave is constrained, several spatial and temporal modes of radiation will be amplified (in a random, uncontrolled manner) at the same time. They will coherently interfere (beat) tu produce large fluctuations. Consider a volume of laser material that has a gain vs frequency profile as showr in Fig. 47. ${ }^{103}$

The number of possible free-space modes available with a typical laser material are $\nu^{\prime}=3 \times 10^{14} \mathrm{~Hz}(\lambda=1 \mu)$ and $\lrcorner y=3 \times 10^{10} \mathrm{~Hz}\left(\Delta y=1 \mathrm{~cm}^{-1}\right)$. Then $\mathrm{N}=2.5 \times 10^{9}$ modes $/ \mathrm{cm}^{3}$, and in a 1 -cm-diam by $10-\mathrm{cm}$-long crystal, $\mathrm{N} \simeq 2.5 \times 10^{10}$ modes. The purpose of an oscillator is to allow only a selected number of these modes to be amplified and to propagate. things occur:

If mirrors are added around the radiating gain medium to form a resonator "cavity." $104-107$ two

1) The spatial directions over which oscillation can occur are limited.

2) The frequencies that can build up in amplitude are fixed by the boundary conditions on the standing wave in the cavity.

The bandwidth is reduced because the photon time in the cavity is increased from $l / c$ to $(/ \mathrm{c}(1-\mathrm{R})$. In addition, the mirrors force a periodic boundary condition, which forces stable electric field modes to occur only at frequency intervals of $\lrcorner \nu=n(c / 20$, where $n=1,2, \ldots$ [see Figs. 48 and 50(a)].

The oscillation condition is best satisfied for the mode closest to the peak of the laser gain vs frequency curve for the medium. Note that for short cavities the spacing between modes $c / 2 C$ is large, and if $J_{1}$ is small, the cavity mode can actually miss the laser gain line: thus oscillation will not occur. This can happen in long-wavelength gas lasers.

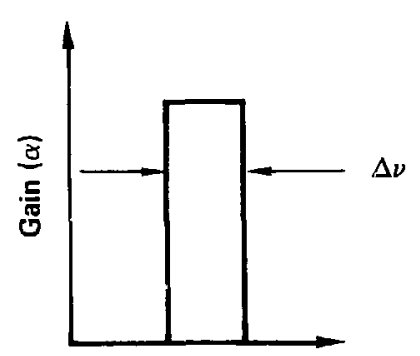

$(v)$

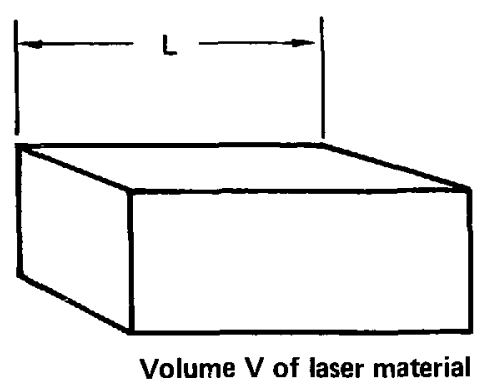

Frequency

Number of definable $E \mathrm{M}$ modes in laser $\mathrm{m}$ zdium above:

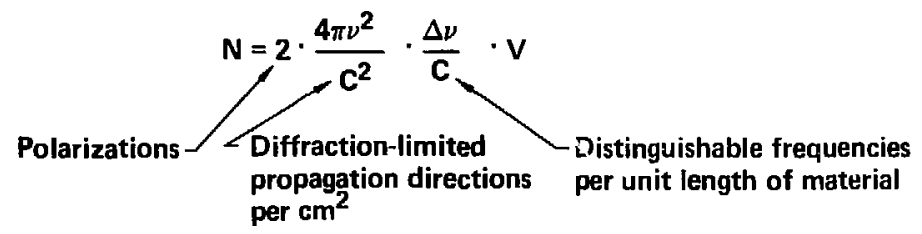

FIG. 47. Modes in a block of laser material. 


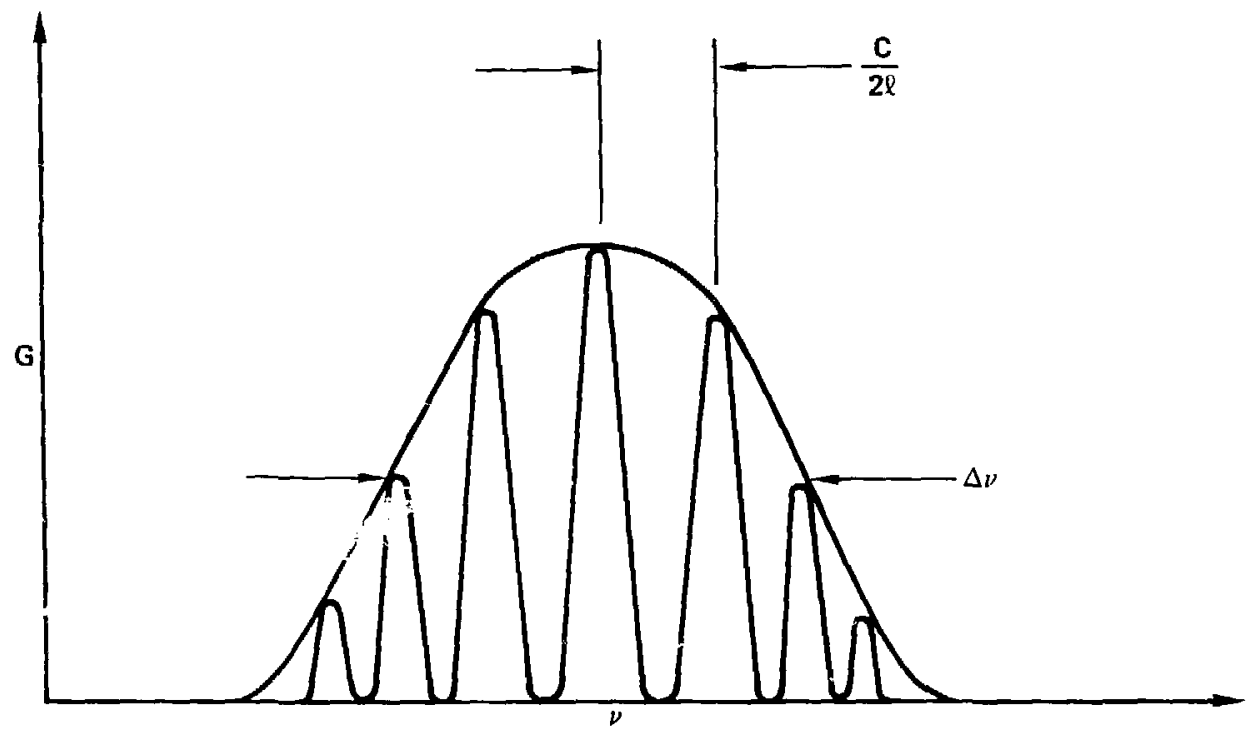

FIG. 48. Gain vs frequency profile $G(v)$ in a cavity is determined by the product of laser transition line-width function $\mathrm{I}(\nu)$ and the cavity reflectivity (or mode structure) $\mathbf{R}(u)$.

The use of a simple 2-mirror cavity does not sufficiently limit the mode structure of an oscillator. When a circulitr aperture is added to limit the spatial mode to the lowest transverse mode, this mode is called TEM $_{00}$ (Transverse Electric Magnetic field), and it is Gaussian in spatial profile [see Fig. 50(b)].

To further control the mode structure, frequency-control elements such as transmission Fabry-Perot etalons are added [Figs, 49 and $50(\mathrm{c})$ ]. This technique is used to further discriminate between natural cavity modes and to ensure that the product of pulsewidth $x$ bandwidth product is near unity for the desired final

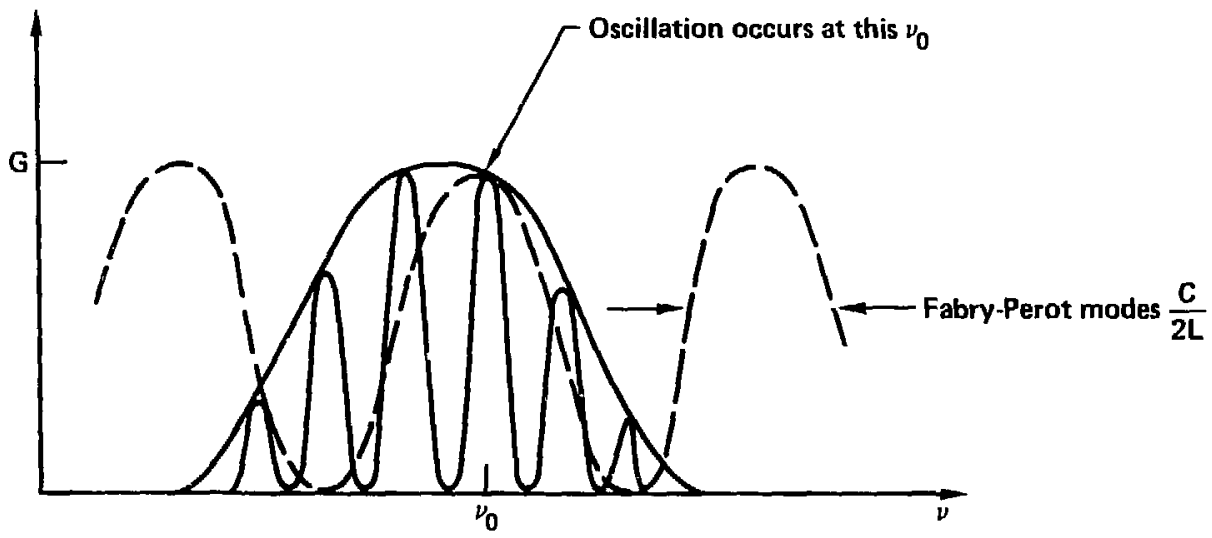

FIG. 49. Lse of a Fabry-Perot etalon in a laser cavity where the etalon thickness $L$ is much shorter than the cavity length f provides additional gain vs frequency modulation. 
$100 \%$

mirror

Gain

(a)

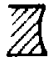

(b)

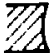

Etalon

(c)

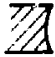

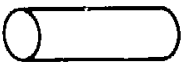

$\mathbf{R} \%$

mirror

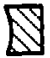

No mode

\section{Aperture}

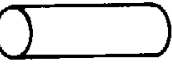

是

月

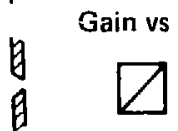

$\mathbb{B}$

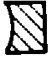

Spatial and frequency mode

Spatial, frequency, and temporal

FI(. 50. Summary of oscillator-mode selection techniques used in bandwidth-limited, mode-locked, and $Q$ switched oscialators.
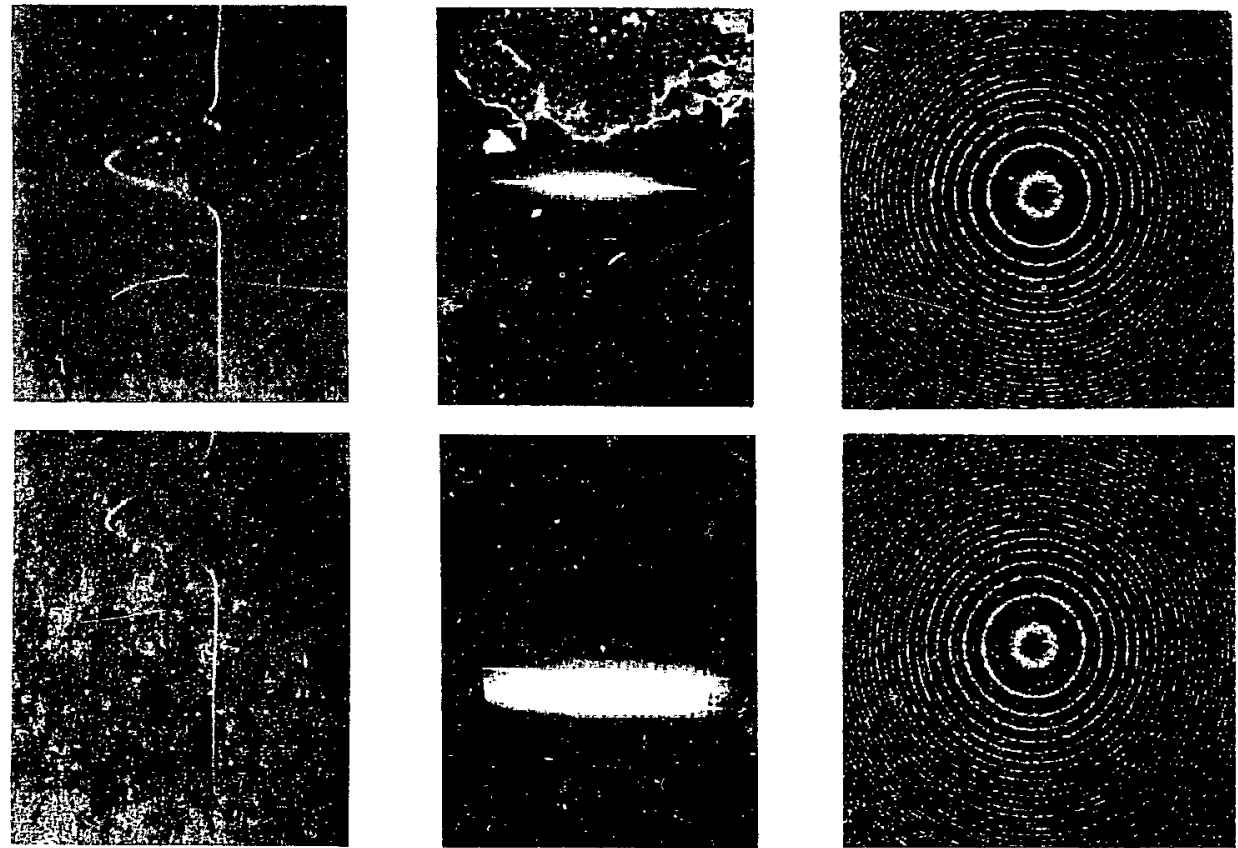

FIf. 51. The top serie; of pictures shows a temporally smooth oscillogram on the left and a smooth streakcamera trace in the middle. ${ }^{108}$ The Fabry-Perot spectrum on the right shows only one mode oscillating. The lower series of pictures shows a slightly skewed oscillogram, extensive modulation on the streak trace, and two competing modes in the Fabry-Perot spectrum. 
output pulse. That is, if a $1-n$ S FWHM Gaussian output pulse is desired, a bandwidth of $\sim 1.10^{-17}$ or $10^{4}$ H/ is arranged. The restriction of the bandwidth guarantees that no accidental secondary niode develops from noise (Fig. 51 ).

In some laser media, the laser gain line is too narrow to provide enough bandwidth to amplify short pulses. The line width must then be modified, usually with pressure, to provide an acceptible gain bandwidth for oscillation and amplification.

Finally, in order to produce a stationary mode in an oscillator calvity, the fields must huild up slonl! enough so that all nonstationary (transient) modes decay. For these reasons a tine-variable lows clement is added to the cavity, as shown in Fig. 50(d). In addition, by modulating the loss element alt at ralle equal to the round-trip cavity time $2 \ell / \mathrm{c}$, bandwidth-limited pulse huild-up is foreed to cycte through the uscillater at the round-trip cavity time $2 f / c$. This technique is ealled "mode-locking," $1114-111$ becatuse several resonatur modes are forced, or locked together, to provide enough bandwidth to sustain anplitication of a pulse "hose bandwidth is wider than the width of the individual resonator modes.

The techniques described above are used extensively in all laser oscillators. In particular. these have been applied to the problem of producing stable pulses from Nd:YAG oscillators for injection into our $\mathrm{Nd}$ :glass laser amplifiers at Livermore. ${ }^{112}$ Examples are shown in Fig. 52.
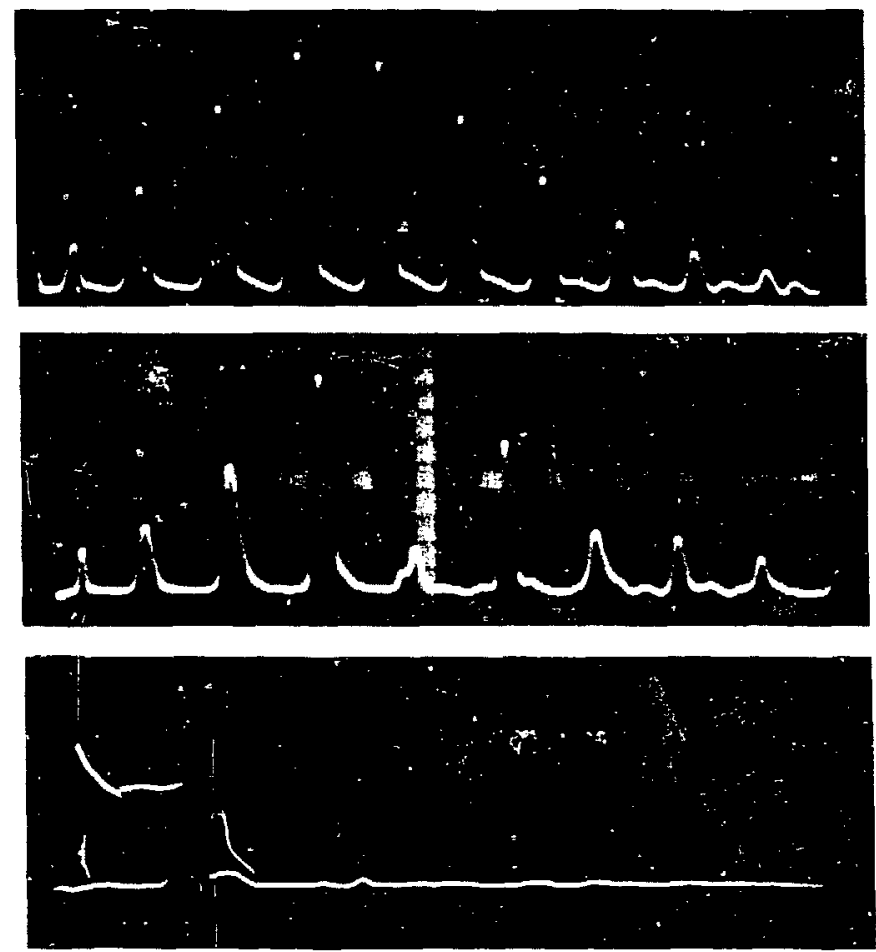

FIG. 52. Top oscillagram shows a train of mode-locked pulses from a modelocked amplifier. The time between them is the round-trip cavity time $2 \mathrm{C} / \mathrm{c}$. The middle trace shows a missing pulse that - $v$ as switched out of the oscillator pulse train and into the amplifier chain. The sottom trace shows the single switched-out pulse ready for pulse amplification. 
Amplifier chain

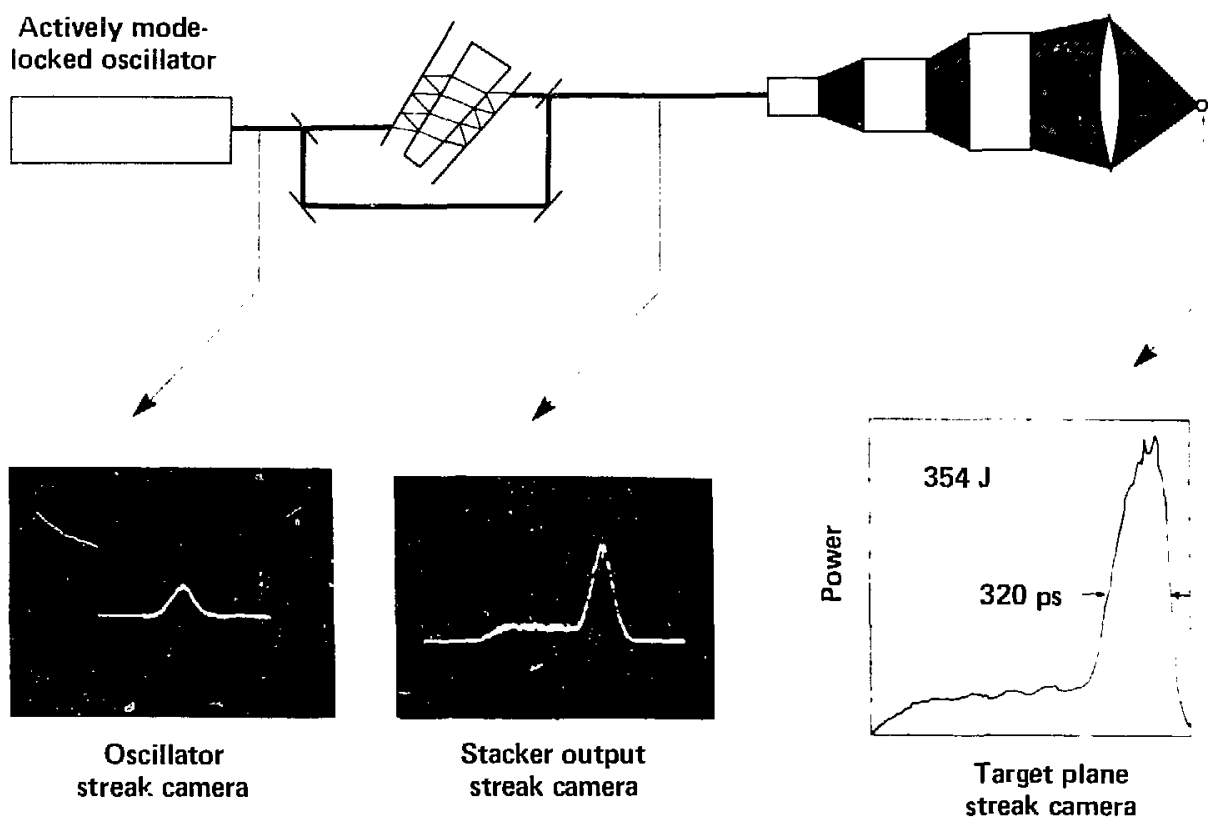

FIG. 53. A phase- and amplitude-stable Gaussian oscillator pulse can be coherently added to itself in an interferometer to obtain time-shaped laser pulses for target experiments.

\section{Summary}

The optical field in the osciliator eavity is allowed to build up gradually, developing a Gausian timehandwidth-linited temporal pulse, a Gaussian spatial profile deternined by the lowest-order diflrationlimited mode, and a radiance determined by stored energy, materials damage limits, and the diffraction limit of the beam diameter. In addition, no secondary pulse trains (prepulses) should be present becaluse the limedependent loss modulation at $26 / \mathrm{e}$ prevents their growth, and no temporal substructure should he present hecause of the limited available bandwidth. Such an oscillator pulse is very well behated and can he cohe:ently arimplified, wo that its radiance and total energy are inereased to levels of interest for plasmia cxperimemtittion. In addition, since the pulse is phase-stable in time and space, it can be conveniently manipulated fur special experiments

For example, it can be spatially truncated to cause its Gaussian transverse shape to fill the initial limiting amplifier apertures. ${ }^{46-98}$ In addition, specific temporal pulse shapes can he "carved out" for special experiment.: and pukes can be coherently added together (Fig. 53) if needed. ${ }^{113.114}$

finally. both the start time and build-up rate in a secondary oscillator can he accurately controlled

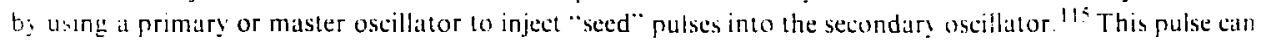
tíner be used for diagnostic or pulse-shaping purposes (Fig. 54) 

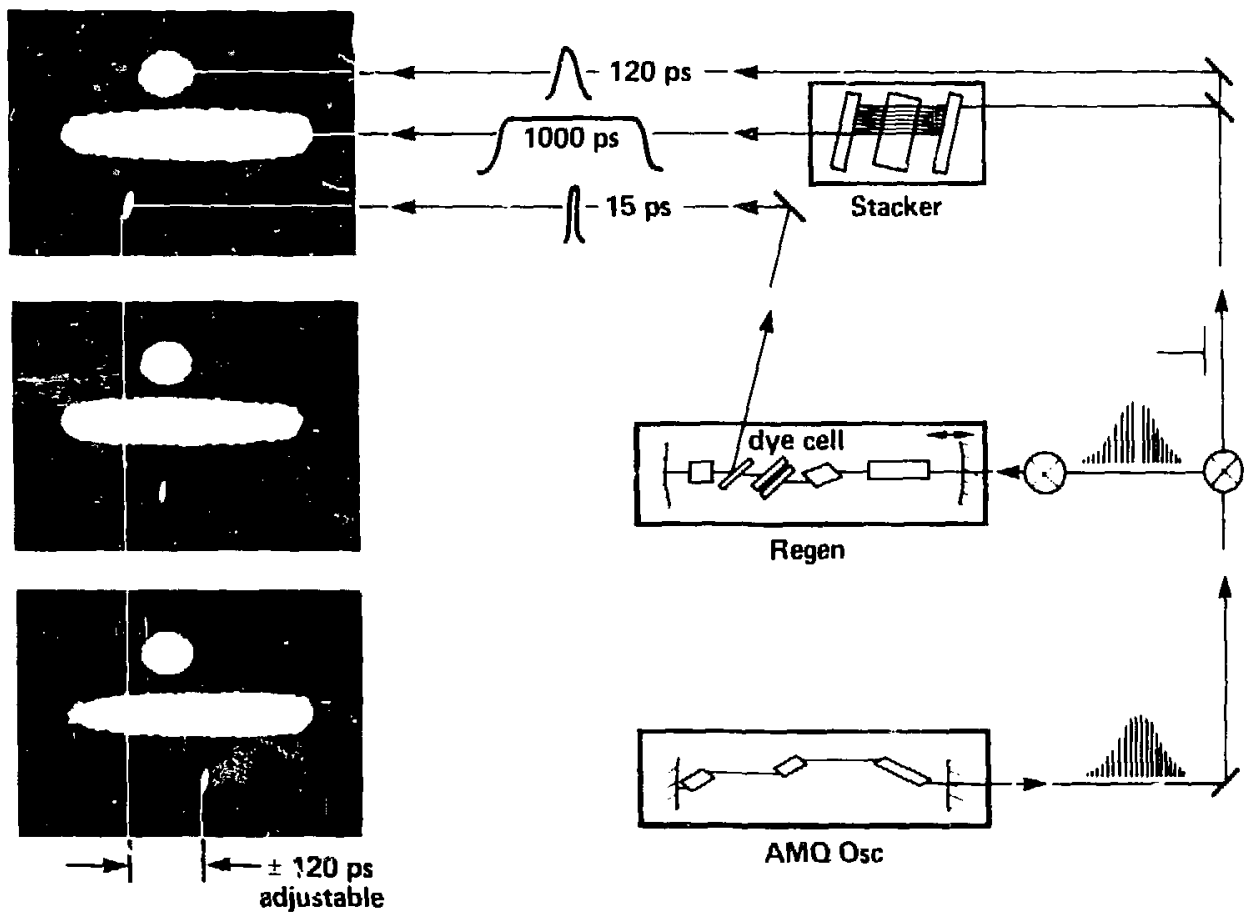

FIG. 54. Regenerative pulse shortening is a technique used to obtain a diagnostic pulse. synchronizable to \pm 5 ps with respect to the master pulse. Coherent pulse stretching provides time-lengthened pulses. AMQ Osc means acoustic mode-locked and Q-switched oscillator.

\section{AMPLIFIERS}

Once the desired optical pulse shape has been generated by the oscillator. it must be amplified to the desired intensity, increasing the heam radiance from that developed in the oscillato: to the level required for target experimentation. This process requires the selection and building of amplifiers that increase the beam radiance level, not reduce it: the amplifiers must also be cost optimized (see Fig. 55).

In the introduction to this report, the types of laser-amplifier gain media available to the laser designer were discussed. The tradeofrs between large and small amplifiers. limits to beam propagation, and stored energy and extractable energy were introduced. In this section the amplifiers themselves and the parameters available to the system designer to modify the amplifying mediun are described. Since a great deal of the cost of a large laser system is in the amplifier. an understanding of the cost vs variable parameters is important.

Virtually all high-power laser amplifiers operate on the principle that the laser-gain medium is a temporary energy store, which integrates "low-grade" electrical energy and stores it in atomic or molecular transitions, until laser-pulse extraction occurs. The exception is the chemical laser, which converts chemical potential energy into an excited intermediate species ready for extraction $t y$ the laser beam. However, chemical lasers for pulsed-amplifier applications require excitation of the interaction region via a trigger signal to set off the reaction. The KrF laser amplifier does not store energy at a very high density, but it does the storage with a high efficiency. Thus techniques using multiple short pulses to extract energy efficiently from the long-pulse KrF system have been devised. 


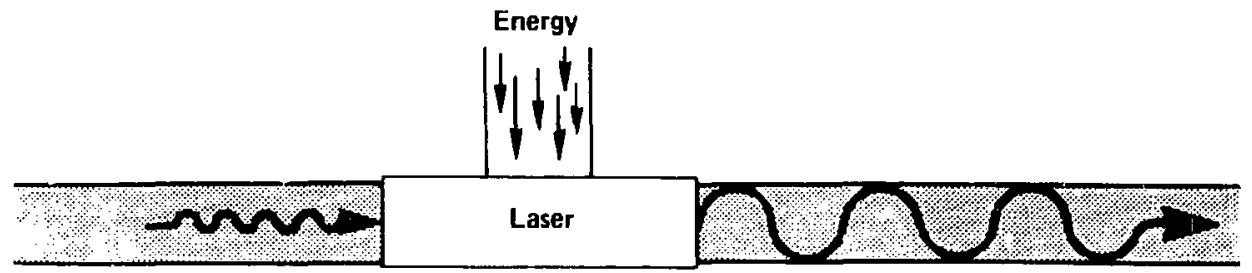

FIG. 55. Laser amplifier uses low-grade electrical energy to prepare atomic or molecular species for coherent light amplification. Typical efficiencies of electrical to photon energy are $0.5-10 \%$. Figures of merit for amplifiers are added energy $/ \mathbf{S}$ or added power/\$ for fixed-aberration contribution.

At present. two sources of excitation energy are of interest to the laser designer-photons and electrons. They are relatively easy to generate and control and most importantly have interaction lengths (energy deposstion lengths) compatible with useful transverse laser dimensions as well as mechanical and electrical enginecring constraints. Since these sourees are used incoherently. they are constrained to excite the medium Iransversely. (Concepts exist to use coherent sources, such as other lasers or high-brightness electron beams that permit longitudinal interattions: however, these will not be discussed here as they are not yet prevalent.) With transverse interaction. a choice of radial illumination or simple two-sided illumination is available.

The radial-pumping geometry in Fig. 56 shows a configuration used most frequently with lowrepetition-rate, photon-pump systems (Nd:glass. $1_{2}$ ). Its main advantage is that a maximum solid angle is available for energy input into the medium. Another advantage is that the radial convergence of pump photon huence lends lo overcome the energy depletion of the pump light as it propagates toward the center, yielding a tnore-uniform gain profile. The right drawing in Fig. 56 shows the geometry used for rapid-flowing gas systems, and for systems transversely excited by high-voltage electrons or by drift electrons accelerated in a onc-dimensional field. Examples are $\mathrm{CO}_{2}$ laser systems and $\mathrm{KrF}$ laser systems.

In all cases the object of the amplifier design is to:

- Store as much energy per unit volume consistent with the excitation source brightness and medium de-excitation due to excited-excited collisions. amplified huorescence depumping. and parasitic thresholds. The objective of a high-stored-energy density arises because amplifier costs are proportional to the volume of the machine.
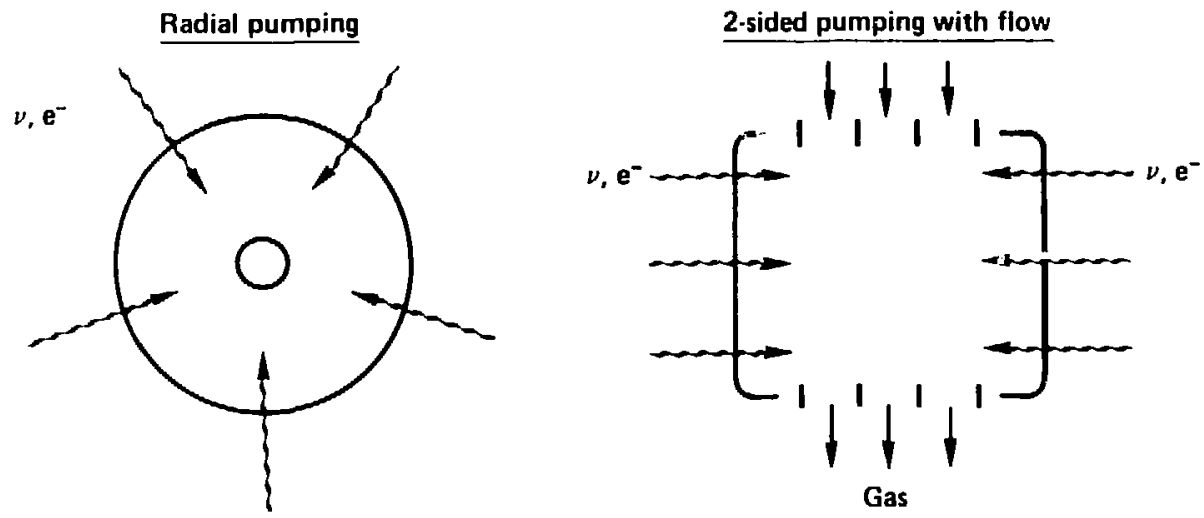

FIG. 56. Trangverse pumping geometries. 
1 Provide an efficiency of excitation consistent with system strategy-that is, minimum total initial cost or minimum operating costs. At present, the predominant economic motivation for all experimental systerns is to reduce the initial capital cost. whereas the motivation for reactor-compatible lasers is to maximize efficiency to reduce toth operating and capitil costs.

- Produce the gain medium with a satisfactory optical quality such that the beam radiance is enhanced. Phase uniformity and gain uniformity are emphasized.

The introduction of an amplifier medium into a propagating laser beam always deteriorates the phase quality of the beam. Modern optical-fabrication techniques permit less than $0.5 \mathrm{~A}$ aberration across the aperture of solid-state amplifiers, and careful attention to gas dynamies permits local density gradients $\mathrm{Jp}_{\mathrm{p}} / \mathrm{p}<10^{-\mathrm{t}}$, which leads to comparable or lower optical aberrations in a gas-laser amplifier. As a result, both total beam energy and the beam radiance increase with the addition of an amplifier, because the added amplifier medium increases the beam radiance with the amplifier gain al a greater rate than the radiance decreases because of aberations. Hence, the radiance inereases to a level of interest for larget experimertaltion. In any experimental situation. the focal quality is a funtion of target design and one can trade off the expense associated with better quality optics against realistic requirenenss associated w ith the laser-target experiments.

Several gain media are being used in fusion lasers. The $\mathrm{Nd}$ :glass laser and the $\mathrm{CO}_{2}$ laser are piven as examples to show the influence of the parameters of the medium on amplifier design.

\section{Nd:GLASS MEDIUM}

The Nd:glass laser is based on the lasing properties of the rare earth neodymium in an amorphous glass host. ${ }^{18-20}$ The concentration of the $\mathrm{Nd}$ ion in the glass host is $-2 \times 10^{20} \mathrm{~cm}^{-3}$. Its decaly rate is 300-600 $\mathrm{s}$. dominated by ion-ion de-excitation. Beciuse the host is amorphous glass. each neodymium ion finds itself in a slightly different site. Thus the line width is an inhomogeneous collection of individual lines. each with $د \lambda \approx 20 \mathrm{~cm}^{-1}$. lead ding to a total line width of $100-200 \mathrm{~cm}^{-1} 21.116$ This line width is very attrattive because it sustains ultrashort pulse propagation. Figure 57 shows the line shape of a huorophosphate laser glass

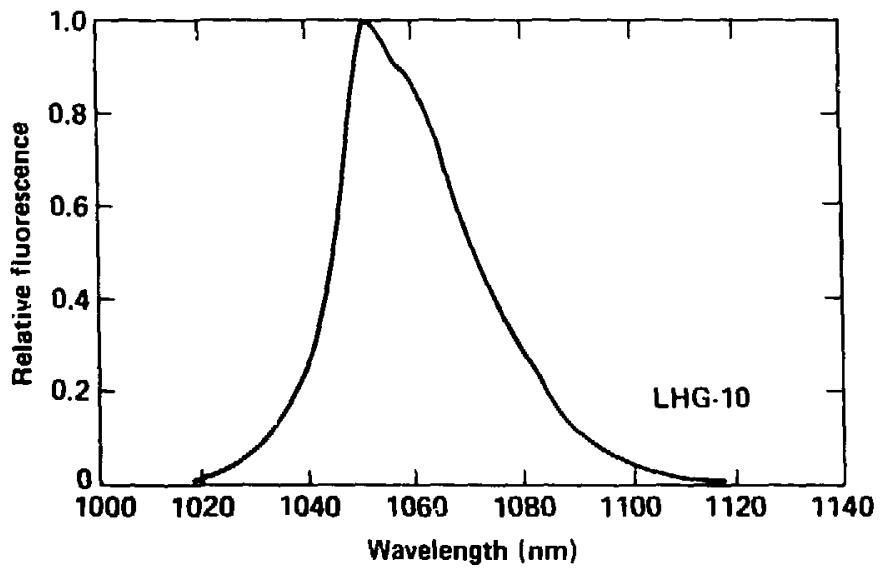

FIG. 57. Fluorescence line shape of iiuorophesphate laser glass. 


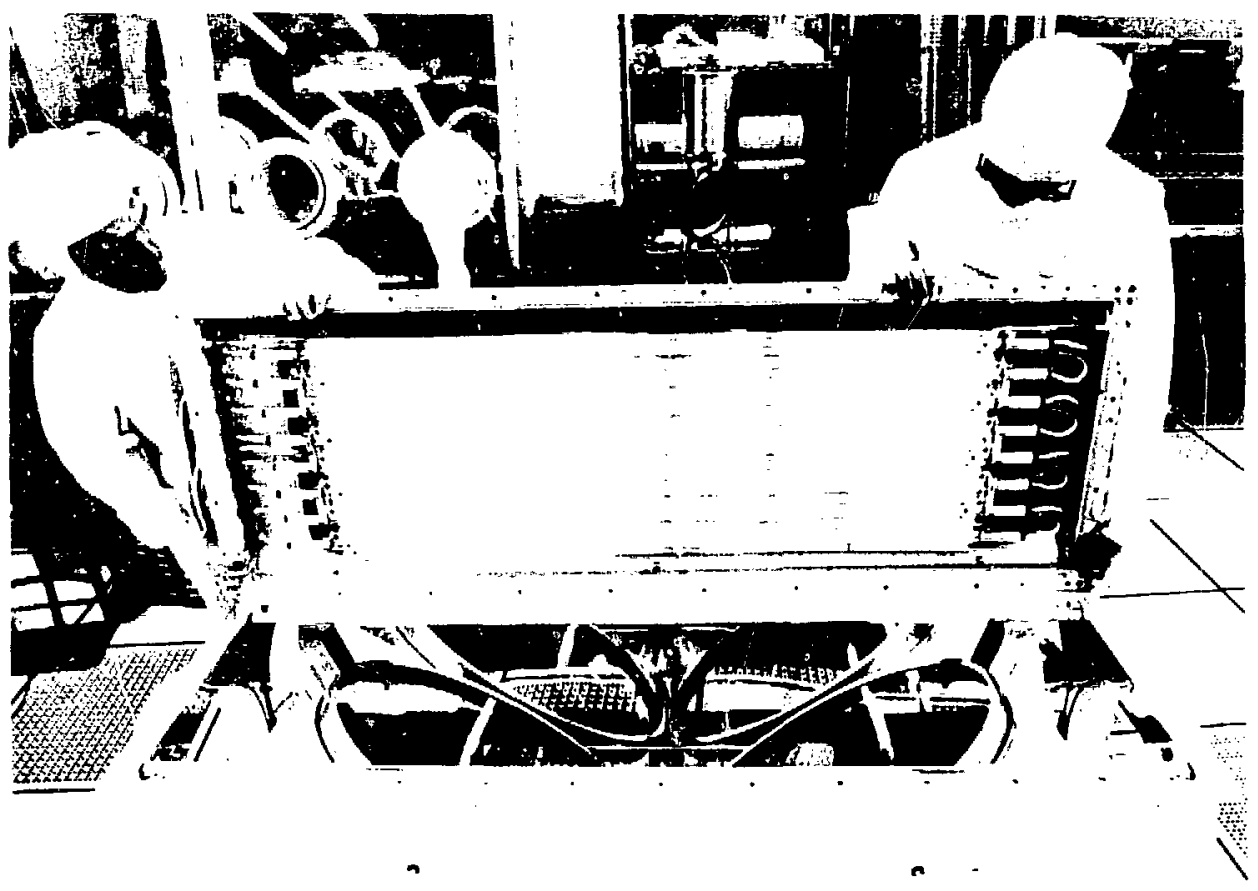

FIG, 58. Nd:glass laser disk ampiifier, for $34 \mathrm{~cm}$ diam laser beam. The small-signal gain ( ${ }^{t+1 .}$ ) is $\sim 1.5$, with $150 \mathrm{~kJ}$ input to the xenon flashlamps. The "planar" pumping geometry yields $\sim 2 \%$ pump efficiency. The laser disks are 3-cm-thick Nd-doped fluorophosphate laser glass.

This system is photon excited, using broadband xenon lashlamp illumination (see Fig. 58). The typical absorption length $(1 / \mathrm{e})$ for the broadband pump photons in laser glass is about $3 \mathrm{~cm}$ for a Nd doping of $2 \times 10^{2 n} \mathrm{~cm}^{-3}$. Absorption length can be varied by increasing or decreasing the $\mathrm{Nd}$ concentration. For beam diameters larger than the typical 3 -cm photon path in $2 \times 10^{20} \mathrm{~cm}^{-3}$-doped (2\%) laser glass, either the doping in a rod must be reduced to increase the photon path or the thin Brewster disk geometry must be
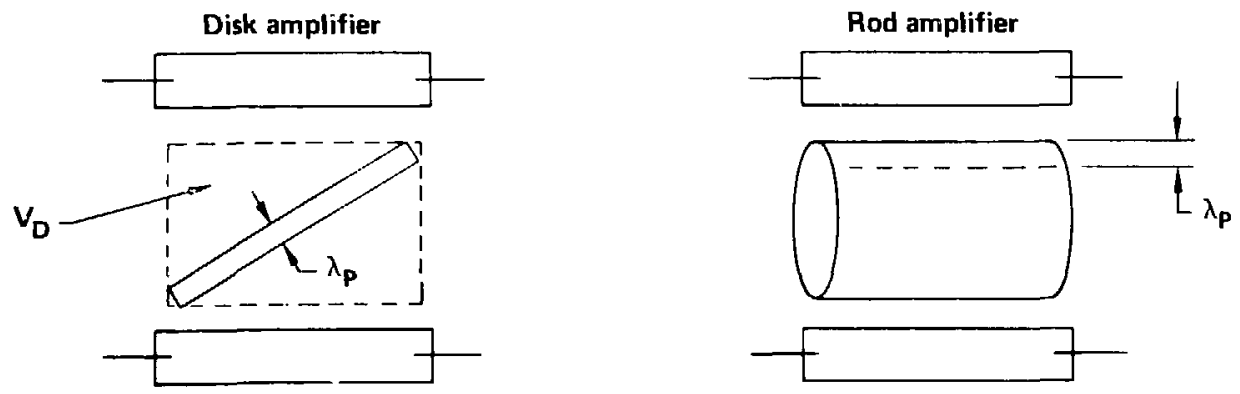

FIG. 59. With transverse optical pumping of glass laser media, the photon mean path $\lambda_{\mathrm{p}}$ determines the size of the amplifier. Disk geometry permits very large diameters, subject to internal parasitic constraints, not uniformity of pumping. 
used. ${ }^{4 \times .117 .118}$ Figure 59 shows the geometry of disks and rods. In a disk, the thackness and Nd doping level are optimized to store energy efficiently and sutisfy propagation constraints.

The glass laser typically stores about $300-500 \mathrm{~J} /$ liter in the disk $\left(0.1 .0 .5 \mathrm{~J} / \mathrm{cm}^{3}\right)$. $1 \mathrm{t}$ is interesting to note that this storage density reduces to 30-50 J/liter of amplifier space. similar to gas-laser systems, because the disk volume is about $1 / 10$ the amplifier volume. The specific gain for a silicate material (Oweni-Illinois ED-2 or Hoya $1 . S G-9 / \mathrm{H}$ ) is $0.149 \mathrm{~cm}^{2} / \mathrm{J}$. Since $0.3-0.5 \mathrm{~J} / \mathrm{cm}^{3}$ is deposited in the inversion, this leads to a gain coefficient of $0.05-0.08 \mathrm{~cm}^{-1}$. The saturation fluence is typically $5 \mathrm{~J} / \mathrm{cm}^{2}$ (at $1 \mathrm{~ns}$ ). This saturation fluence is altractive for pulsed lasers $(>\mid \mathrm{ns}$ ) because it permits reasonable energy extraction at fluence levels below surface-damage thresholds.

Small, high-gain amplifiers (preamplifiers) are aimost always needed to boost the oscillator output to the level of $\sim 1 \mathrm{~J} / \mathrm{cm}^{2}$ over an approximate $2-\mathrm{cm}$ transverse dimension. This beam size and energy level are convenient for measurement. the intensity is within conservative propagation limits, and the size is ready for injection into a power-ampliner chain. This can be done by transversely pumping a glass rod. ${ }^{119}$ The photon absorption length is comparable to the rod diameter; thus, uniform high gains $(\sim 100)$ are available. The rod gain is chosen to keep the gain within bounds imposed by stability constraints.

An at lractive feature of $\mathrm{Nd}$ in glass is that the $\mathrm{Nd}$-ion-gain storage time is long ( $300 \mu \mathrm{s})$, permitting the use of low-cost, straightforward electrical-pulse discharge technology. Since electrica!-energy storage is a large fraction of system costs, this is a positive factor in reducing initial capital in building a research laser.

The dynamics of glass pumping are as follows. ${ }^{120}$ The pumping occurs from the periphery, and the total Nd population that can be excited is constrained by the radiance of the xenon Hashlamps. The lamps radiate $50 \mathrm{~J} / \mathrm{cm}^{2}$ of broadband light. This is divided by the packing fraction of lamps, which is about 2 . Thus the cylinder around the glass gain media emits $\sim 25 \mathrm{~J} / \mathrm{cm}^{2}$ with the $80 \%$ efficiency of a xenon lamp. Typical transfer efficiences from lamp-to-stored-inversion in a disk laser glass are $1.5 \%$; the $25 \mathrm{~J} / \mathrm{cm}^{2}$ radiated leads to $\sim 0.4 \mathrm{~J} / \mathrm{cm}^{3}$ stored in 3-cm-thick disks. This results in a small-signal gain coefficient of $\mathrm{a}=0.06 \mathrm{~cm}^{-1}$. For a gain of exp $(\alpha \ell)=10$ per amplifier stage, $38 \mathrm{~cm}$ of gain path is needed. This requires $32 \mathrm{em}$ of plale thickness. Since the beam propagates in a skew direction through the plates, the total path is 1.2 times the disk thickness. This path can be composed of eight 4-cm-thick disks. With a gain coeficient $a=0.06 \mathrm{~cm}^{-1}$, the largest transverse dimension $($ (which is $\sim 2 X$ beam diam in the disk) is defined by the disk parasitic constraint of ix $\geq 3-4.4^{45,47}$ Thus $f \leqslant 67 \mathrm{~cm}$ and the beam diameter would be $33 \mathrm{~cm}$. This disk amplifier wouic be divided into four amplifiers of two disks each, as shown in Fig. 58.

As a result of the favorable stimulated-emission cross section, the reasonable energy-storage efficiency and cost, the high optical quality, and the short-pulse amplification capability, this laser system has found wide acceptance as an experimental tool. For a fusion-:eactor laser, however, it suffers from low efficiency and a low thermal recovery rate.

\section{$\mathrm{CO}_{2}$ GAS MEDIUM}

The molecular gas $\mathrm{CO}_{2}$ has relevant laser levels that are low-lying vibrational excitations at $\sim 0.1-0.2 \mathrm{eV}\left(200 \mathrm{~cm}^{-1}\right)$ above the ground level. ${ }^{22,23}$ The $\mathrm{CO}_{2}$ is mixed with $\mathrm{N}_{2}$ and He gas to enhance its efficiency. The $\mathrm{CO}_{2}$ laser levels are easily populated by de-excitation from high-lying vibrational transitions in $\mathrm{N}_{2}$ or $\mathrm{CO}_{2}$. Both the $\mathrm{CO}_{2}$ and the $\mathrm{N}_{2}$ are directly excited by electrons moving through the partially ioni.ed laser medium. ${ }^{121.122}$ Because $\mathrm{e}^{-}$or $\mathrm{UV}$ photon ionization can be used to generate free electrons, the transverse configuration is especially useful for applying a sustainer electric field of $3-5 \mathrm{kV} / \mathrm{cm}$ to accelerate the electrons to excite the $\mathrm{N}_{2}$ and $\mathrm{CO}_{2}$ vibrational excitations. ${ }^{123-126} \mathrm{~A}$ sketch of the geometry is shown in Fig. 60 .

The advantage of $\mathrm{CO}_{2}$ excitation by electrons is that the medium can be scaled to very large transverse dimensions, because the range of pre-iunizing electron can be very long. The transverse diameter is limited only by parasitic modes associated with very large transverse gain lengths. The range of electrons in $\mathrm{CO}_{2}: \mathrm{He}: \mathrm{N}_{2}$ gas mixtures is $\sim 1 \mathrm{~m}$ at $1 \mathrm{MeV}$. The actual laser excitation is provided by the "sustainer" field. ${ }^{126}$ The energy deposition rate from the sustainer field is $\mathrm{V} \cdot \mathrm{I} \cdot \mathrm{Il}\left(4 \mathrm{kV} / \mathrm{cm} \mathrm{atm} \cdot 10 \mathrm{~A} / \mathrm{cm}^{2} \cdot 3 \times 10^{-6} \mathrm{~s}\right)$ or $120 \mathrm{~J} /$ liter. Because of the partition of energy in the rotational levels, $\sim 15 \mathrm{~J} /$ liter can be extracted with a single 


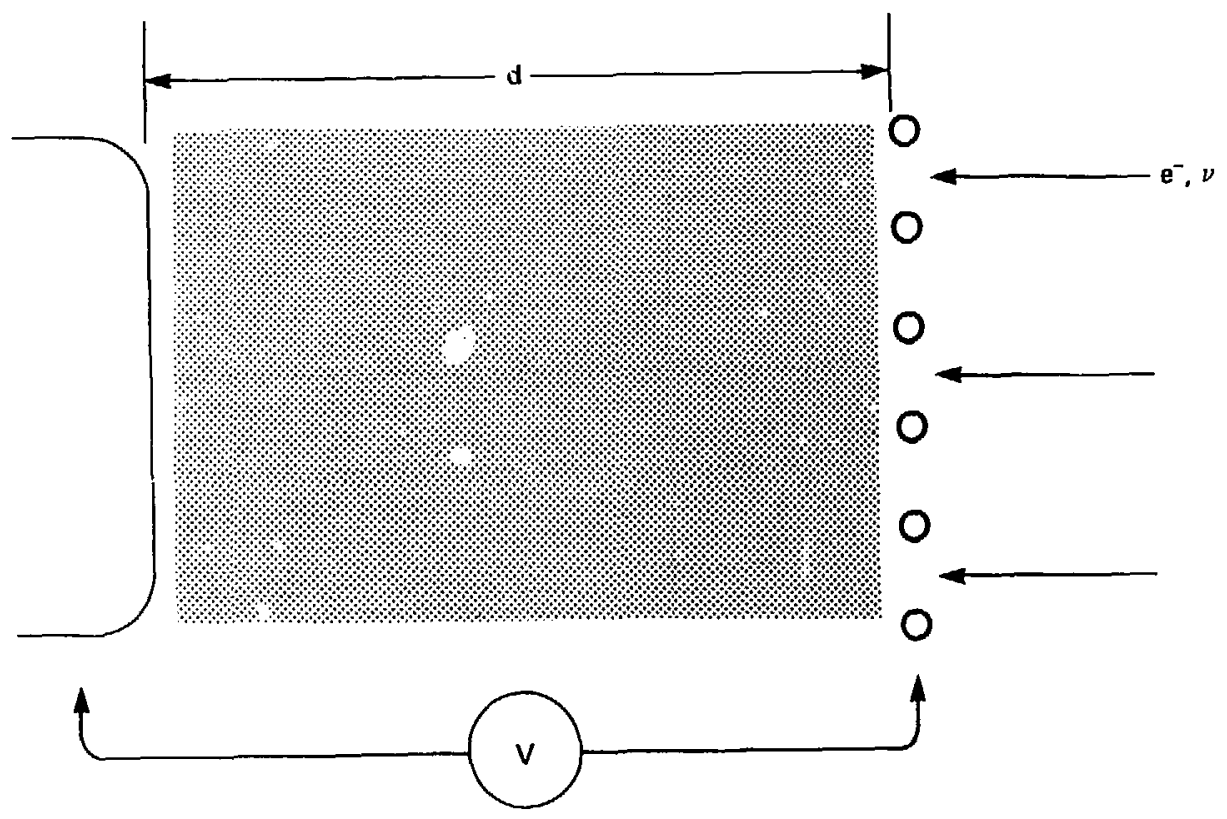

FIC. 60. Electrons (ar photons) jonize the laser gas medium. A sustainer field, represented by the voltage generator $V$, causes a curreat to flow between the anode on the left and a grid on the right.

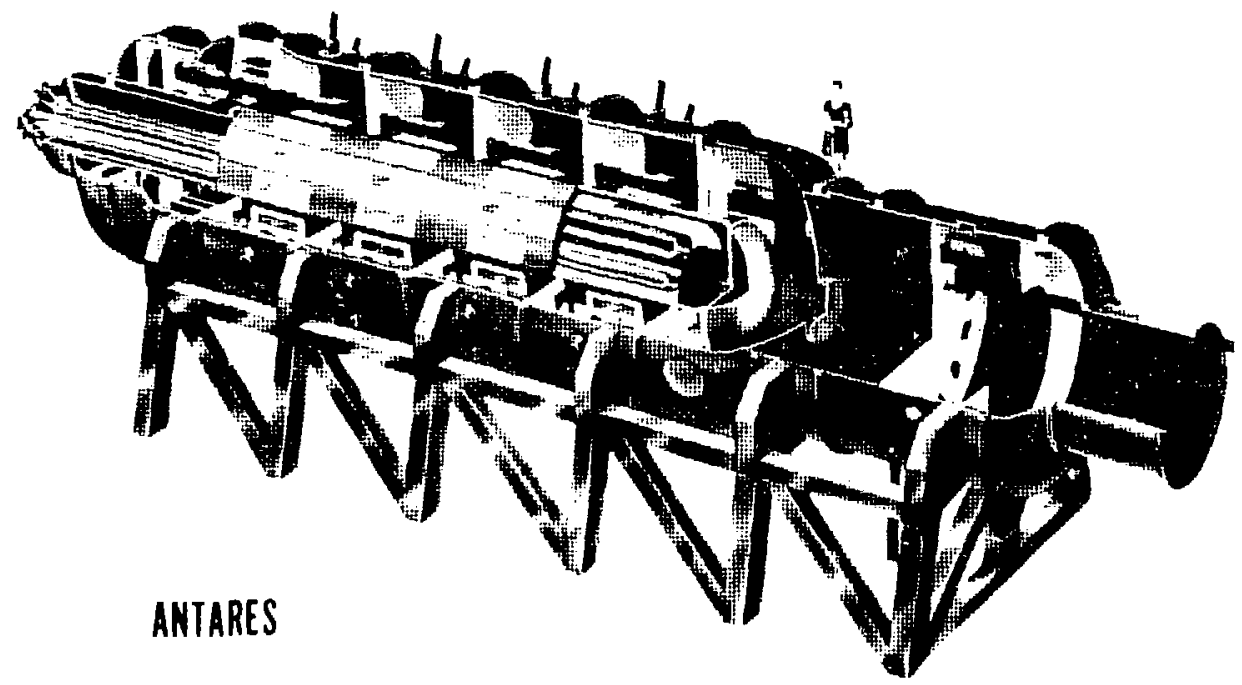

Fici. 61. Power amplifier in the Antares laser system at LASL. The electron beam is directed radially outward into the annular gas region where the sustainer field is applied. 
$\sim 1$ ns pulse. Typical gains of $0.04 \mathrm{~cm}^{-1}$ are achieved in 1 atm gas. The saturation fluence is $\sim 0.10 \mathrm{~J} / \mathrm{cm}^{2}$, well below material damage thresholds of $\sim 5 \mathrm{~J} / \mathrm{cm}^{2}$ at $10.6 \mu \mathrm{m}$. The gain line width is complex, consisting of an entire series of rotational lines around the P-branch transition at $10.4 \mu \mathrm{m}$ and around the R-branch transition at $9.6 \mu \mathrm{m}$, at moderate pressures. At high pressure the lines merge together.

This laser system is scalable, efficient, and moderate in cost. It provides a convenient source of longwavelength, high-quality radiation for target-irradiation experiments.

Figure 61 shows the power amplifier in the Antares laser system at the Los Alamos Scientific Laboratories. The electron gun projects ionizing electrons radially outwards into the annular laser medium. The sustainer field supplies energy to the laser gas.

\section{SPATIAL FILTERS AND RELAYS}

Imaging elements are required in a laser chain to control the divergence and diameter of a latier beant as it is intensified. The primary element is ihe astronomical telescope, shown in Fig. 62. It projects i real image of the input beam farther down the chain, thus overcoming the natural diffraction of the laser beam. This device performs several functions. It expands the heam. projects the beam farther down the chain, reduces ASE, and spatially filters the beam when an aperture is added at the focus between the two lenses. The spatialfiltering function and the expansion functions have been discussed. ${ }^{80.81}$ The purpose of relaying is to mintain a high fill factor by imaging one laser aperture to the next and to prevent diffraction rings from forming ${ }^{919} \mathrm{An}$ example is shown in Fig. 63.

The optimum length for the spatial-filter relay is determined by its imuging properties and is independent of the diameter of the filtering pinhole. This imaging property is shown in Fig. 64. It shows two adjacent filters in an optical train, in which the space between the filters is assumed to be filled with listercomponent hardware. Optimum use of apertures is achieved if plane $Z_{N}$ (input lens of filter $N$ ) is imaged onto plane $Z_{N}+1$ (input lens of filter $N+1$ ). In addition, locations along the beam path preceding location $Z$, will be imaged onto a location defined in $\mathrm{L}_{N}$. Using the simple formulas for a two-lens system. the correct length of the filter is found to be $\mathrm{L}_{\mathrm{f}}^{\text {optimem }}=\mathrm{L}_{N} / \mathrm{M}$, where $\mathrm{L}_{\mathrm{N}}=$ length of the optical-component train between adjucent filters and $M=$ magnification of the filter. The magnification of the fiter is the ratio of the diameter of the beam exiting the filter to the diameter of the beam entering it, $M=D_{2} / D_{1}$. (Note that this ratio is determined solely by amplifier staging considerations.) Therefore, the optimum length of a spatial filter is uniquely determined by the diameter of the clear aperture of components on either side of it (i.e., its magnification) and the length of the optical train between it and the filter immediately following it.

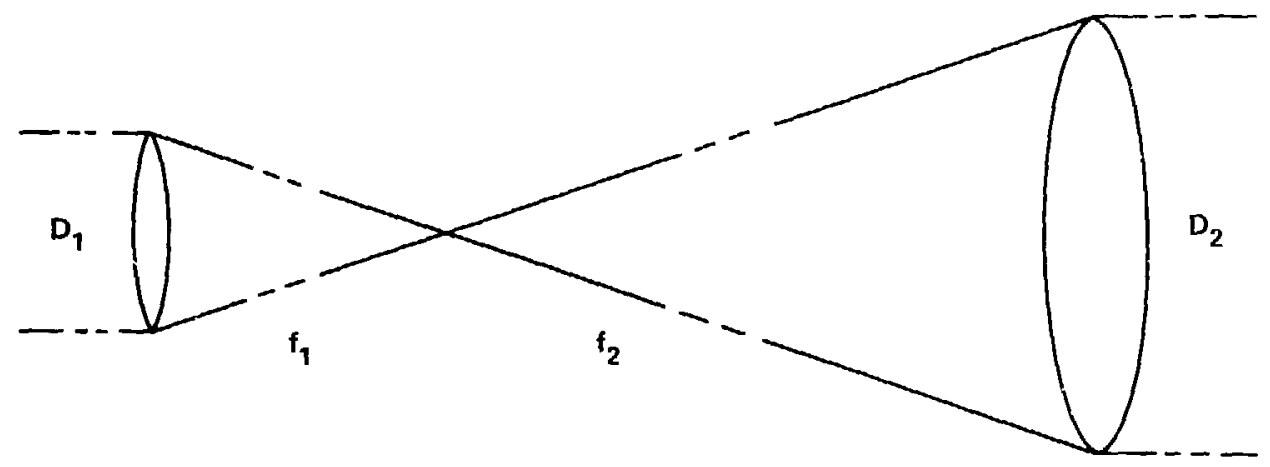

FIG. 62. Astronomical telescopes project real images of the beam and the laser apertures from the oscillator tc the target focus lens. 


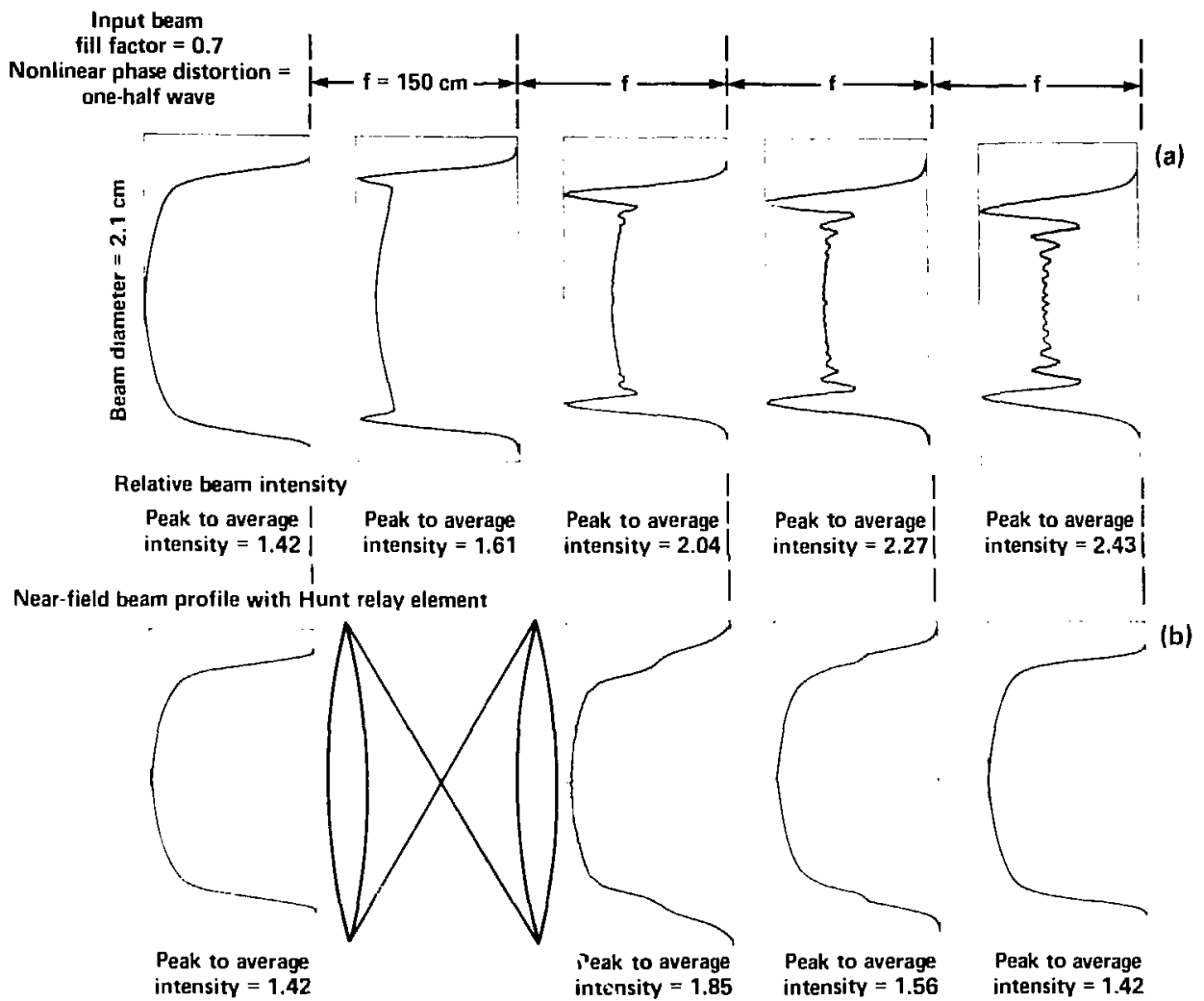

FIG. 63. Beam propagation-diffraction and self-focusing (calculations performed with ARTEMIS computer code) (a) Combination of diffaction wffects and nonlinear phase distortions produce large intensity gradients. With further propagation in nonlinear l:edium self-focusing enhances these gradients and reduces focusable power (b) The use of relay elements reduce: toffraction effects and thereby minimizes growth of intensity gradients due to nonlinear phase distortions.

A second consideration for spatial-filter length is to prevent plasma formation in the pinhole. ${ }^{127}$ No closure occurs if the laser pulse is short compared to the pinhole closure time. For long pulses, $\tau_{2}>R_{\text {pinhole }} / v_{\text {plasma }}$ the intensity of the beam focal spot at the pinhole edge must be less than the threshold for plasma formation or the pinhole must be much larger than $v_{p l a s m a} t_{\text {puise }}$

An example of the effect of relaying and spatial filtering on beam propagation is shown in Fig. 65 . Figure 60 shows a $20-\mathrm{cm}$ spatial filter used on the Argus laser system.

In Fig. 65, each line shows the propagation of a laser beam, from left to right, urder different conditions. All of the initial conditions are the same: a small spot $(0.8 \mathrm{~mm}$ diam.) obscures part of the beam and causes diffraction ripples. The beam profile is shown at propagation locations of $0,1,2,3,4$ and $5 \mathrm{~m}$. If a modifying element is present, such as the obscuration spot, a lens, or a nonlinear element, the element was located at the location shown and the beam profile is shown immediately upon exiting that element. In line (a) the evolution of the beam and the difiraction ripples from a $0.8 \mathrm{~mm}$ obscuration are shown as they propagate $5 \mathrm{~m}$ down a laser chain. 


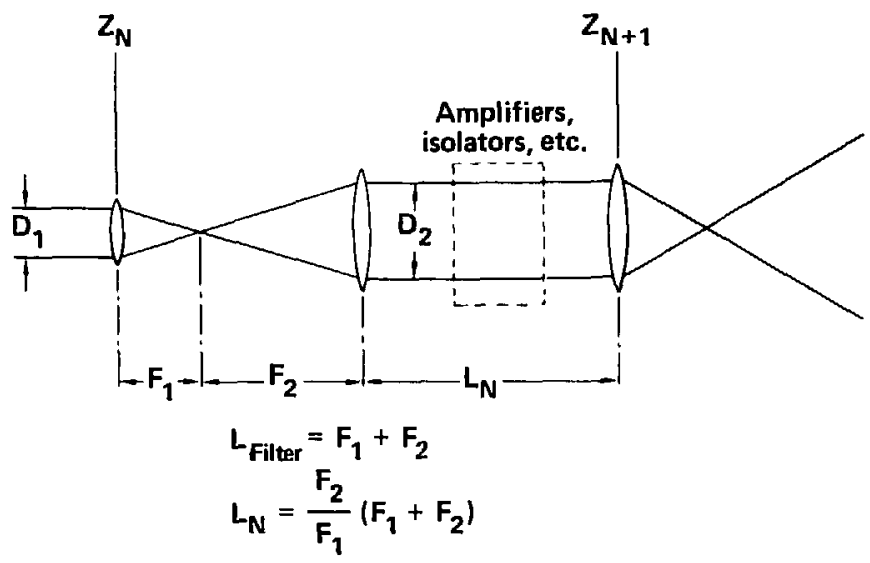

FIG. 64. Selection of relay length. The beam and aperture at location $Z_{N}$ is imaged at location $\mathrm{Z}_{\mathrm{N}+1}$.

In (b) an element with an intensity-dependent index of refraction is placed at the $1 \mathrm{~m}$ location. The average nonlinear phase retardation for the radial envelope of the laser beam was $\sim 1 / 2$ wave $(B=3 \mathrm{rad})$. Notice the increase in the peak intensity of the beam compared to (a) which has no nonlinear materials. In (c) the effect of a simple relay is demonstrated (no nonlinear elements). The image at $0 \mathrm{~m}$ is reimaged to $4 \mathrm{~m}$ by the 1-to-1 image relay.

In (d) a nonlinear element is added directly upstream from the relay input. The phase aberration contributed by this element causes severe distortion downstream and prevents image formation of the $0 \mathrm{~m}$ position onto the $4 \mathrm{~m}$ position. However, the image at $1 \mathrm{~m}$ (at the lens, immediately after the nonlinear element) is reimaged to the $5 \mathrm{~m}$ location because no additional nonlinear aberrations are present. In real lasers we image from the input of one relay spatial filter to the input of the next spatial filter for this reason.

In (e) a pinhole is added to remove the high-frequency ripples associated with the small obscurations and subsequent nonlinear enhancement of its high spatial frequencies. The beam exiting the relay/spatial filter is suitable for subsequent laser amplification and propagation.

\section{ISOLATORS}

Isolators act as optical diodes, permitting light to pass in one direction but not in the other. They are used to prevent back-reflected laser light from re-entering the small diameter stages. This is accomplished by either the Faraday effect (the polarization plane of the light is rotated by an amount proportional to the strength of a magnetic field applied along the direction of propagation), or by the Pockels effect (the polarization plane is rotated by an amount proportional to the material length times the electric field). The Faraday effect is particularly attractive because of its scalability to large diam ${ }^{* 4}$ ers. ${ }^{128,1^{2} y}$ With it, forward-propagating, linearly polarized light can be rotated $45^{\circ}$ in a right-hand sense with respect to the direction of the magnetic field. Thus, the plane of polarization of any back-reflected light ; vill be perpendicular to that of the initial beam and can be rejected by a polarizer. The Faraday effect occurs ir almost all glasses, particularly in paramagnetic glasses.

The Pockels effect occurs in ferro-electric crystals such as potassium dihydrogen phosphate (K DP) and its isomorphs. ${ }^{130.131}$ It achieves isolation by simply rotating the beam polarization for the requisite time period ( $\sim 10 \mathrm{~ns})$ and allowing the pulse through the crossed polarizers. 


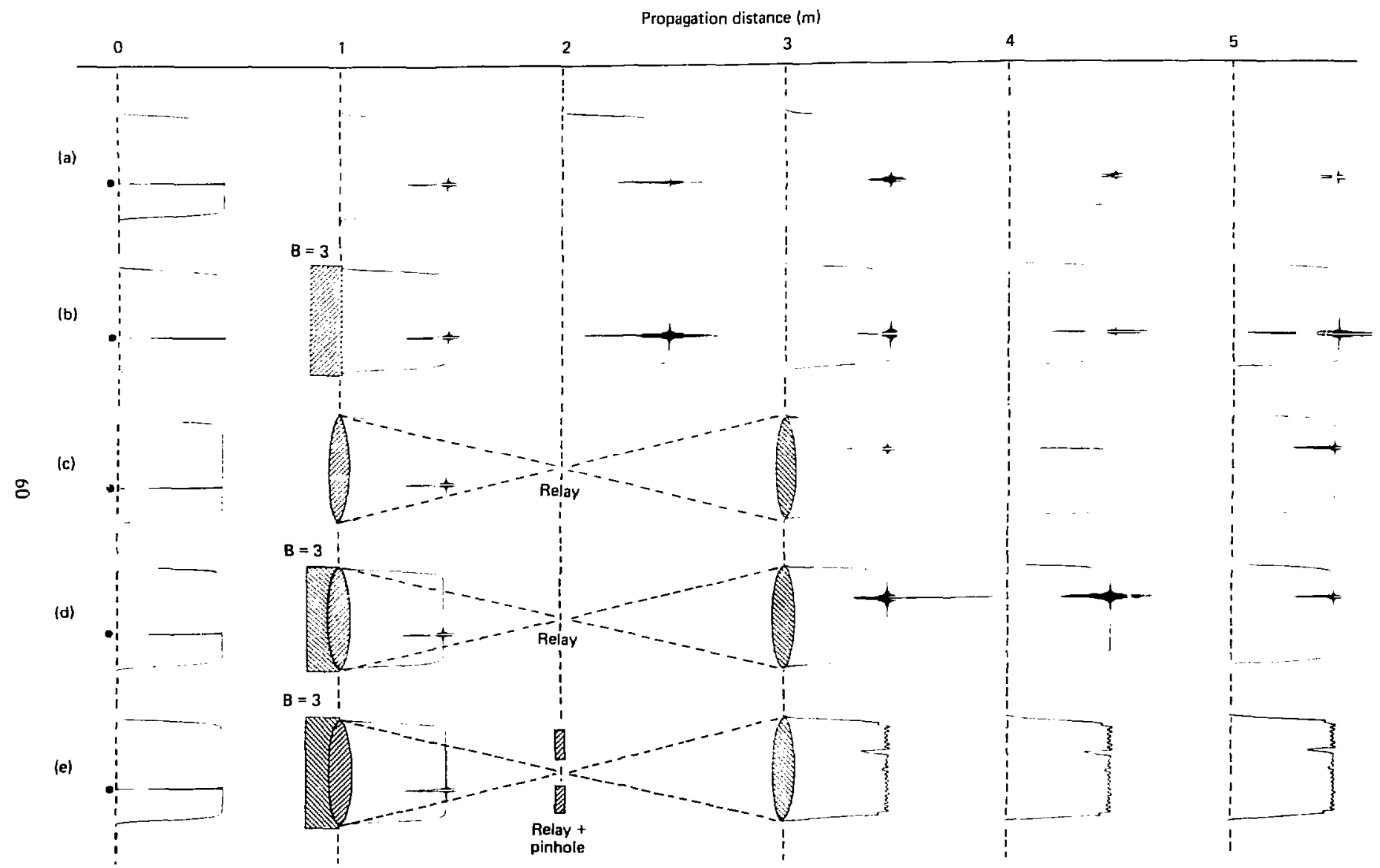

FIG. 65. Propagation of a $1 \mu$ wavelength $14-\mathrm{cm}$-diam constant-intensity laser beum with conditions of linear and nonlinear propagation, spatial filtering, and imaging relaying. (Note: the initial obscuration is not drawn to scale.) 


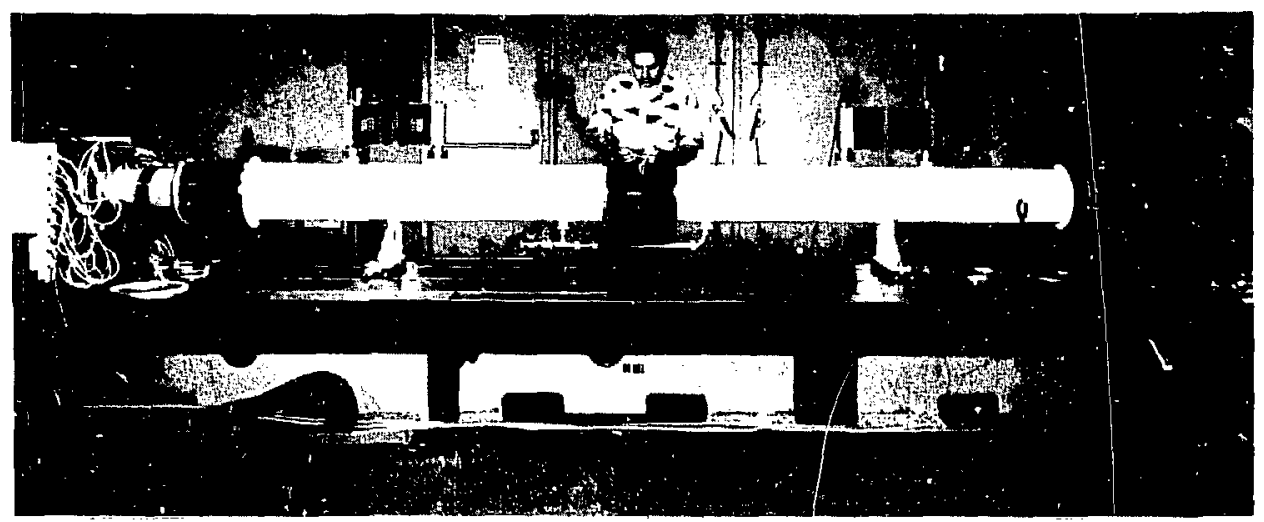

FIG. 66. Argus laser 20-cm-diam spatial filter. The pinhole section is directly in front of the man.

TABLE 10. Faraday rotator material properties at $1.06 \mu \mathrm{m}$.

\begin{tabular}{|c|c|c|c|c|c|}
\hline Designation & Inn & $\begin{array}{l}\text { Verdet coefficient } \\
\text { V(min/Oe cm })\end{array}$ & $\begin{array}{l}\text { Refractive } \\
\text { index }\end{array}$ & $\left(\times 10^{-13}\right.$ esu $)$ & $\begin{array}{c}\text { Loss } \\
\left(\mathrm{cml}^{-1}\right)\end{array}$ \\
\hline SFS6 & - & 0.029 & -1.8 & $\times 2.0$ & 0.007 \\
\hline FR4 & $\mathrm{Ce}$ & 0.031 & 1.551 & 1.95 & 0.005 \\
\hline EY-1 & $\mathbf{T b}$ & 0.037 & 1.610 & 1.77 & 0.005 \\
\hline FR5 & Tb & 0.069 & 1.678 & 2.0 .3 & 0.005 \\
\hline
\end{tabular}

The degree of isolation required in a laser chain can be calculated for a given gain per stage $G$. If the reflected light returns along the same path as the original forward beam. and the intensity $\left(\mathrm{W} / \mathrm{cm}^{2}\right)$ or fluence $\left(\mathrm{J} / \mathrm{cm}^{2}\right)$ nowhere exceeds that in the outgoing beam, then it can be shown that the extinction ratio required for polarizers is $\mathrm{G}^{-4}$ per stage and the uniformity of rotation required for the rotators is $\mathrm{G}^{-2}$. Thus, a system consisting of successive amplifier stages with gains of 3.2 requires a polarization extinction of $100: 1(-20 \mathrm{~dB})$ for each stage. In Faraday rotators, the magnetic-field uniformity must be such that, after one pass, less than $1 \%{ }_{\text {. }}$ of the light is of the "wrong" polarization at any (transverse) position of the beam. This translates into a magnetic-field uniformity of about $10 \%$. The system requirements are thus $-20 \mathrm{~dB}$ extinction ratio per stage for polarizers and $-10 \mathrm{~dB}$ uniformity for rotators.

Glass for Faraday rotators must have short optical path length tu minimize nonlinear effects, absorption, and the effects of residual and thermal birefrigence. Hence. a high Verdet constunt $V$ is desirible. This has led to the development of paramagnetic terbium glasses. ${ }^{57.132}$ Material properties for pertinent glasses are shown in Table 10.

The essential features of a Faraday rotator are shown schematically in Fig. 67.

\section{SUMMARY}

Some of the properties of the important components required for laser-chain construction were summarized in this section. The engineering of the comporents into a laser system is essential for successful target irradiation. A complete discussion of the engineering requirements of these systems can be found in the reports of the laboratories engaged in laser-fusion research and in reports of contractual research sponsored by the U.S. Department of Defense, which are available from NTIC. 


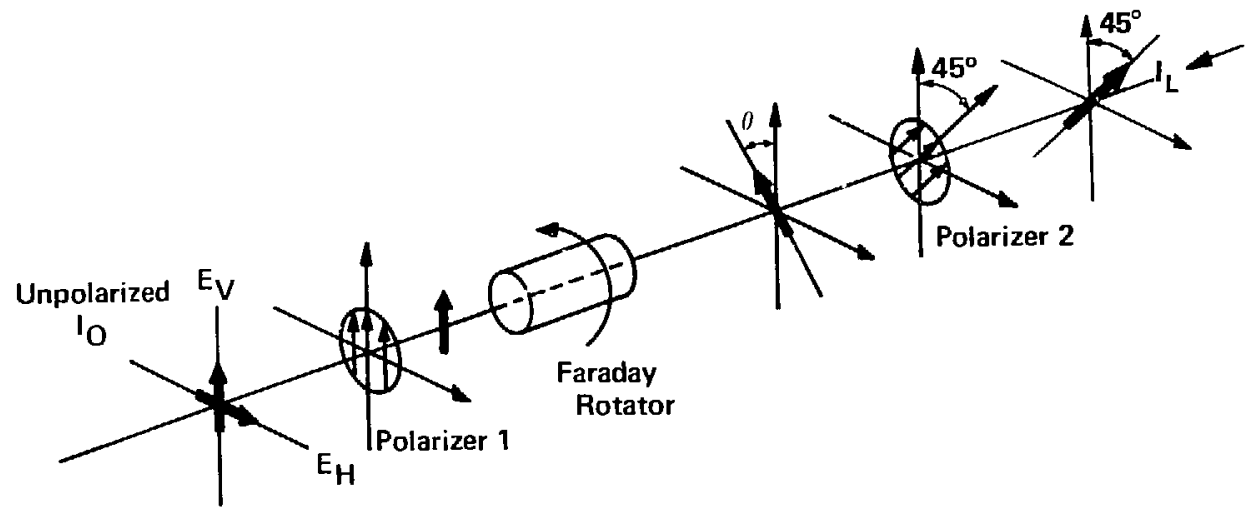

FIG. 67. Inpolarized light reflected back from a target on the left is partially rejected by polarizer 1 and completely rejccted by polzrizer 2 . Laser light propagating from a laser stage at the right passes through polarizer 2 and is rotated to pass through polarizer 1 , then goes to the target.

\section{LASER DIAGNOSTICS}

In the laser-plasma interaction problem, a natural separation occ : at the interface between the incoming laser beam and the plasma. While this interaction regime is not strictly defined, a common experimental approach is to specify the initial-beam focal conditions at the target-vacuum interface. A model is then attempted, in a self-consistent fashion, of the time evolution of the interaction, knowing the time evolution of the laser pulse and the physics of the light-plasma interactions. ${ }^{133}$ The complete description of this interaction is the subject of a great deal of research. Present practice is to provide as complete a description of the laser input as possible, in order to set the initial conditions between laser and target. Then, subsequent comparison between target performance and target simulations can be used to distinguish between competing models.

It is difficult to completely diagnose the laser performance because it is time consuming, expensive, and probably unnecessary. The instruments for measuring the laser interaction are, in many cases, as close to the stete-of-the-art of development as are the laser and targets. The best diagnostic would be a motion picture of the beam-target interaction with picoseccnd framing over focal intensities from $\sim 10^{10} \mathrm{~W} / \mathrm{cm}^{2}$ (at plasma initiation) to peak intensities of $\sim 10^{15} \mathrm{~W} / \mathrm{cm}^{2}$, and down the trailing edge of the pulse. The spatial resolution should be on the order of the diffraction spot. Such complete dynamic range and information content is not presently possible. With a minimal set of diagnostics it is possible to be sure that the laser focal spot is reasonably well behaved and that all subsequent target behavior is due to the "mysteries" of plasma response. not laser performance. A typical laser-target diagnostics set is shown in Fig. 68. The techniques shown in these notes are those used at Lawrence Livermore Laboratory for the $1.06-\mu \mathrm{m}$ laser systems. Other laboratories use similar techniques but with important variations because of special problems caused by the availability of special optics and diagnostics at other wavelengths.

\section{VITAL MEASUREMENTS}

Proper laser operation is ascertained by monitoring at the points described in Fig. 68. Accurate measurements of the beam energy along the laser chain from oscillator to focal lens, when correlated with beam images and focusable energy measurements, give the operator confidence that the laser chain is operating correctly. A correlation between easily obtained laser-diaguostic indicators and measured laser 


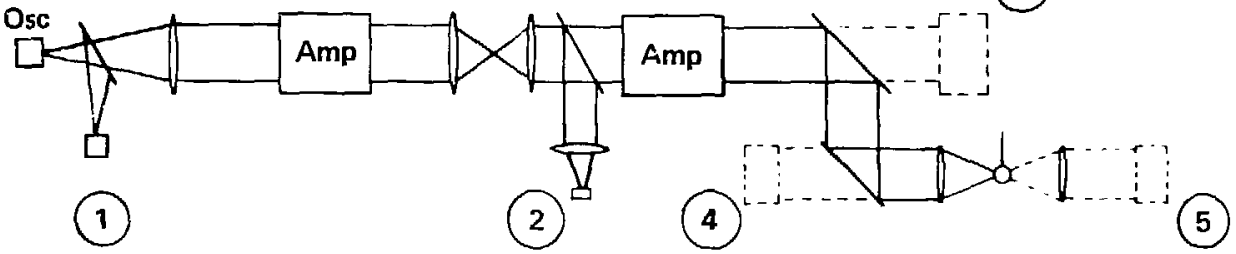

FIG. 68. Diagnostics are : (1) Oscillator performance and chain input beam quality. The oscillator pulse duration, spatial shape at the input to the chain, and the input energy. (Prepulses are usually too small to measure here.) (2) Bearn quality at an intermediate chain location. The energy level is used to determine amplifier chain performance, time-integrated beam photographs to determine the quality of image. Damage spots, self-focusing, apodization, etc., are observed. (3) Incident-beam diagnostic station. The beam quality at the exit of the laser chain is measured here. These measurements include energy, the pulse shape in time, a tine-integrated near-field beam image to determine propagation quality, a far-field diagnostic location, and a diagnostic location to image chain points back toward the uxcillator for pinhole alignment. $(4,5)$ Return-beam diagnostic location. This location is used to image both the target and the opposice beam's fecal quality. The reflected beam energy, spectra. and time-evolution diagnostics are located here. Also located here are automatic laser-target pointing sensors.

focal performance is one of the most important activities in laser-system maintenance, because the resources or time to constantly verify laser focal quality generally are not available.

The most important diagnostics for the laser-target irradiation are the "far-field" diagrostics 134 shown in Fig. 69. They are usually acr . olished by using secondary optical systems to measure the focal spot on the target (1), and to stimulate $\mathrm{tl}$ ical spot with a secondary far-field focal system (2).

(2)

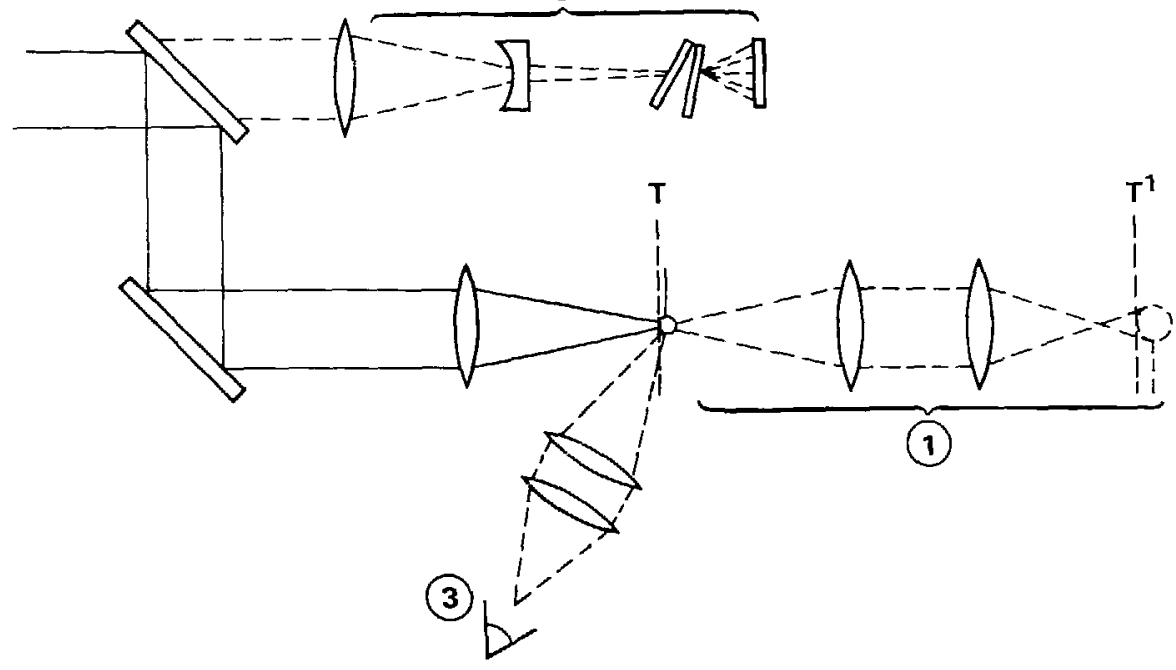

FIG. 69, Schematic of ineident-beam diagnostic package (2), and two target viewing packages (1) and (3). 
Each of these systems works by using an objective lens to image or to re-image an thiect onto an imaging device such as a vidicon for real-time observation, or onto sensitive film for pulse recording. System (1) (Fig. 69) re-imuges plane $T$, usually the front target surface, onto a vidicon. Using this device the laser focal size is set, and if the target is transparent, the target is positioned with respect to the beam and the beam size is adjusted (see Fig, 70). A low-level cw laser, coincident with the pulsed oscillator beam, is used for most of this work, because it has been found ${ }^{135}$ that the $\mathrm{cw}$-beam focal properties simulate the aberration and focal properties of the fully pulsed laser chain satisfactorily.

For diagnostic system (1) in Fig. 69, the primary objecive lens is usually the focusing lens on the oppusite incoming laser beam. For the lens to serve as an imaging objective for the target plane $T$. it must be used at its designed focus and within its somewhat narrow field of view. The secondary lens system is chosen to obfain the desired magnification.

System (2) (Fig. 69) is similar, except that it is designed to diagnose the incident laser beam (IBD diagnostic) by directly imaging a fraction of the incoming laser beam. The beam passes through a turning mirror and is focused onto an imaging device or through a pinhole and into a calorimeter. This diagnostic then measures "far-field" or focal-plane energy distribution and, when directed onto a streak-camera slit, farfield time duratior. at this location can also be measured. At this location prepulse diagnosis is also performed. for this diagnostic to accurately record full-power laser performance, its effective image position must be the same as that of the laser focal spot on the target. Figure 71 shows the Argus incident-beam system.

Finally, a third system (3) (Fig. 69) has also been used ${ }^{136}$ to record the image of a focused beam on a larget by imaging the seattered light from the focal spot onto an imaging sensor. This technique is sometimes useful for examining the focal distribution on an opaque target, but it is difficult to implement on transparent targets.

The accuracy with which systems (1). (2), or (3) replicate the actual radiation distribution at the target depends on the quality of the optical components in each diagnostic optical train. A great deal of care must be taken to be certain that the images obtained with each of these systems correspond to the actual distribution.

In a typical setup at L.I.L. on a well-instrumented laser system such as Argus, the following measurements are made:

- Uscillator

Oscillat or pulase-train shape and intensity.

Oscillator suitch-out pulse energy.

Streak camera image of input pulse to chain.

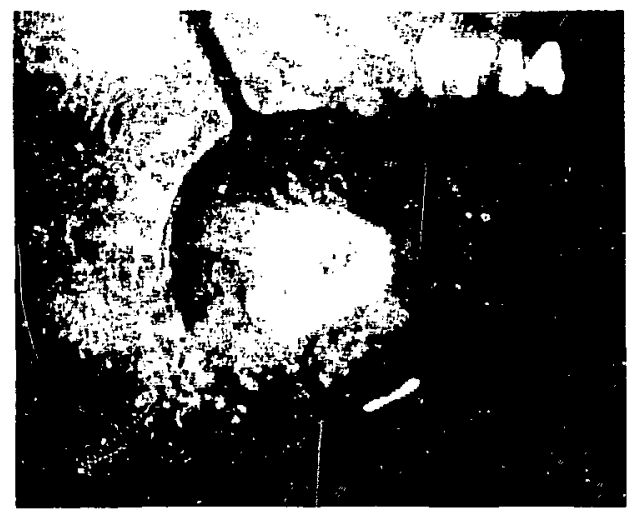

FlC. 70. Target ball ( $100 \mu \mathrm{m}$ diam ) showing position of incūming laser beam. 


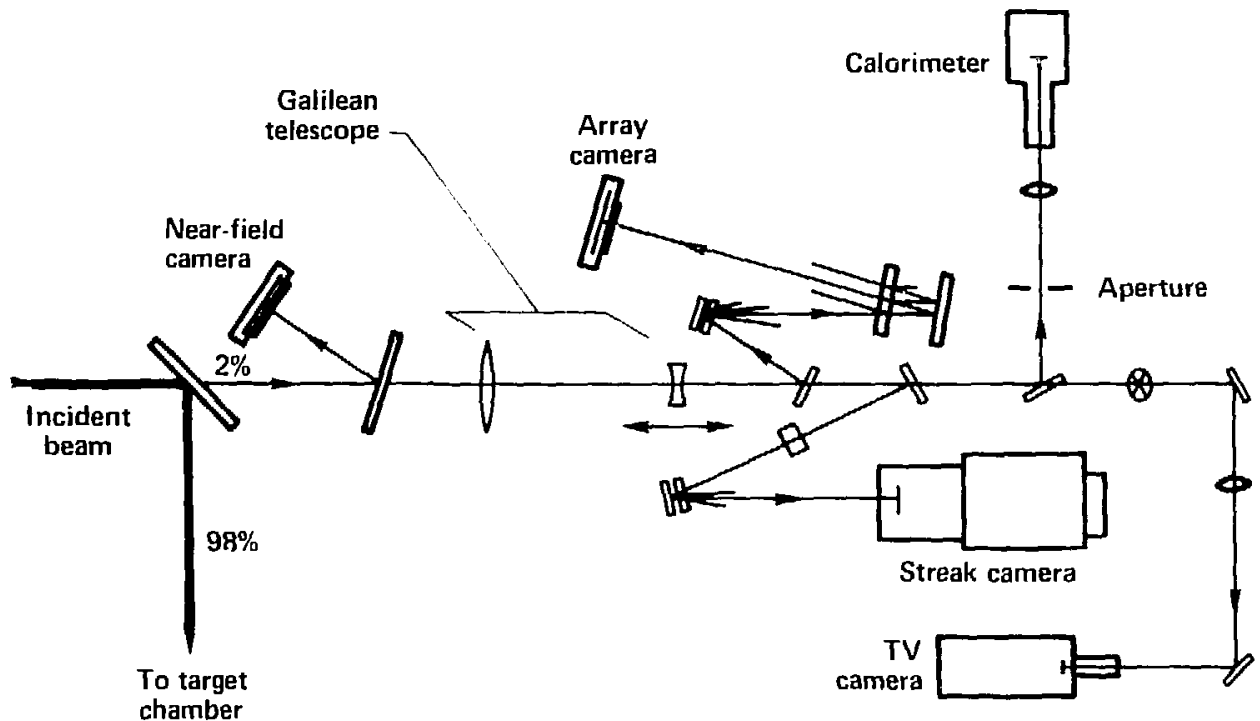

FIG. 71. Argus incident-beam diagnostic system.
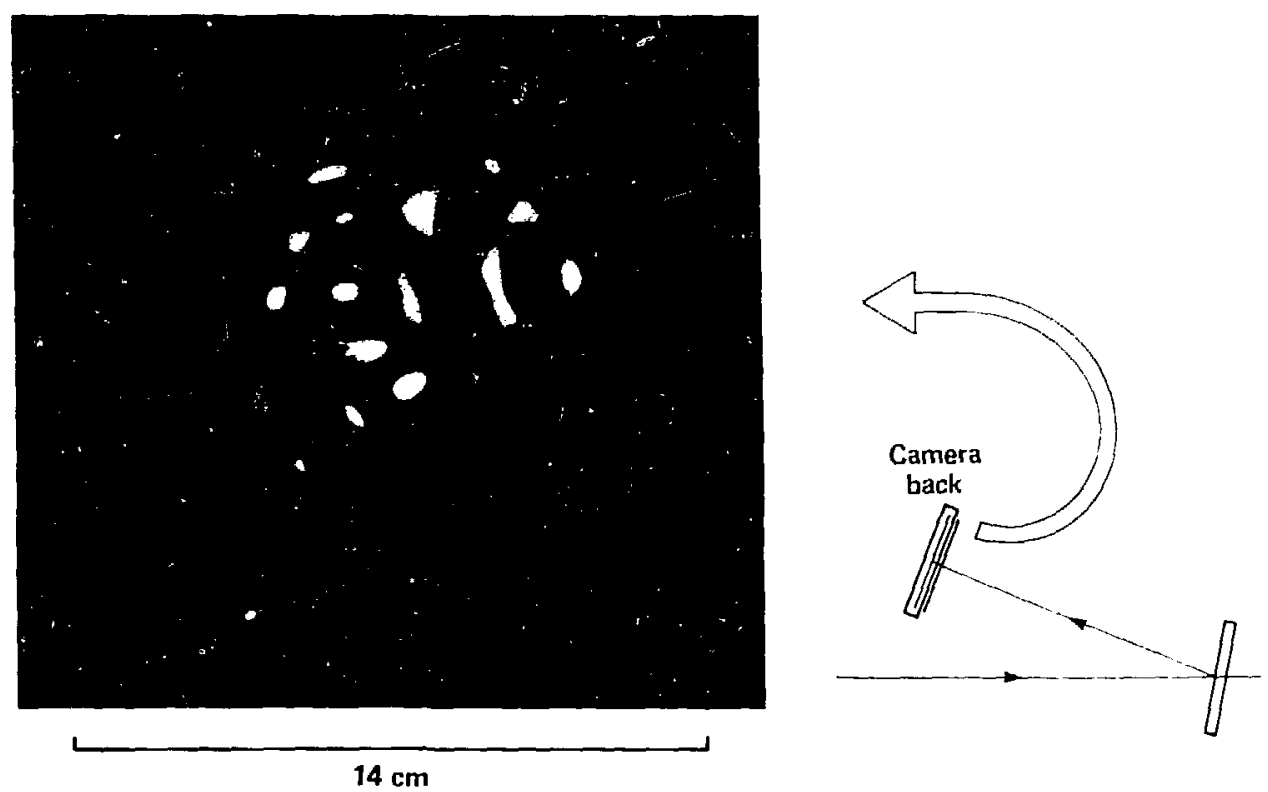

FIC. 72. Near-field camera-laser-energy distribution. 
- Laser chain

Energy at 4-cm rod, prepulse energy.

Energy and beam profile (see Fig. 72 ) at exit from 10-cm disk amplifier.

- Incident-beam diagnostic

Laser-output energy.

Laser-output pulse shape (100:I dynamic range).

Laser far-field energy, through pinhole.

Laser focal pattern at various locations in front of, at, and behind target plane.

Prepulse diagnostic-70 dB ahead of pulse peak.

- Return-beam diagnostic package

Reflected energy.

Reflected target image.

Reflected pulse shape.

\section{LASER SYSTEMS}

The assemblage of laser components and diagnostics for target experimentation is a laser-target system. ${ }^{137-141}$ The effectiveness of this system as measured by single-shot performance (TW or $\mathrm{kJ}$ )/ $\$$. by the number of successful shots per day. by the cost of uperation. or by overall electrical efficiency, is inliuenced greatly by the manner in which the system is assembled.

Present research laser-target systems are designed to ma ximize performance and to minimize capital cost. The number of experiments per day and the cost of operations. while recessary considerations. have been of lesser importance than initial cost. Electrical efficiency is not important for research lasers. except to reduce the capital cost of the laser electrical power system. As fusion lasers make the transition from very low-dutycycle systems to reactor drivers, emphasis is shifting to overall efficiency and low maintenance costs. with capital cost being a lesser consideration.

Primary system considerations are:

Laser architecture.

Fluence and aberration limits.

Component efficiency.

Quality of engineering and physics design

Laser architecture influences the overall size (length and diameter) of the system. thus fixing the costper-beam aperture and strongly influencing related costs such as building space. maintenance equipment. etc. Fluence-limits fix the aperture size required to meet a given power level and the amount of amplification required to bring a beam from the oscillator output to a fluence-limited output area. Aberration limits constrain the length and optical quality of the laser medium. Component cost, especially amplification, is generally proportional to diameter squared and to electrical efficiency. In many components, such as the amplifiers and Faraday rotators, the electrical-system cost is two thirds of the total component cost: thus efficiency influences the component cost. Finally, good engineering design layout, conservative operational levels, and proper execution of this system consistent with laser-physics constraints are essential in meeting objectives and having a system that can be reliably operated.

\section{GENERIC SYSTEMS}

Figure 73 shows three generic forms of system architecture. The master-osciliator power amplifier (MOPA) system is the most common fusion-laser system and is used exclusively at LLL. A low-level bandwidth-limited pulse is injected into pulse amplifiers. which increase the pulse energy to levels consistent with propagation constraints. By sclection of the rate of beam expansion and amplifier medium. reasonable extraction of stored amplifier energy occurs within the constraints of fluence limitations. The disadvantage of 
this system type is that a large number of amplifier sizes and optics are required and many amplifiers are not operated at full-beam fluence: thus their stored energy is not efficiently extracted. The positive attributes are that this system design is relatively straightforward, the propagation issues are clear, the beam travel distance is minimal, and the linear aberrations of the beams are averaged as the beam traverses the many laser components. Finally. it is the only system (until recently) that has been physically realizable with the present understanding of laser design and components. As a resuit. most operating fusion-laser systems are of this design. Examples are the Livermore Argus and Shiva lasers.

The second and tbird types of laser systems, shown in Fig. 73, are variations of the multipass geometry. $1+2,1+3$ The primary object of these geometries is to extract as much of the stored energy as possible from a single amplifier system, thus avoiding the need for a multiplicity of components and inefficiently used amplifiers. The regenerative liser system in Fig. 73 is, with some variation, the usual oscillator geometry used for generating low-level pulses. The problems associated with the off-axis multipass system are the long propagation paths required to reduce bearn vignetting at the amplifier aperture and the need to use largediameter components to make proper use of economics of scale. The major problem with the switched regenerative system is the attainment of a large-diameter optical switch that has a rapid switching time (consistent with cavity length), low leakage to prevent prepulse. and low-loss optical components. In both multipass geometries. the repetitive cycling of the uptical beam through the same components laads to cumulative aberration buildup. The second and third systems are especially sensitive to intracavity losses, because unless the round-trip loss is low, the amplifier energy that is transferred to the laser optical field is in turn deposited in the cavity losses and not stored in the optisal field for eventual deposition on the targ-: The switch in the regenerative system is quite prone to excess loss.

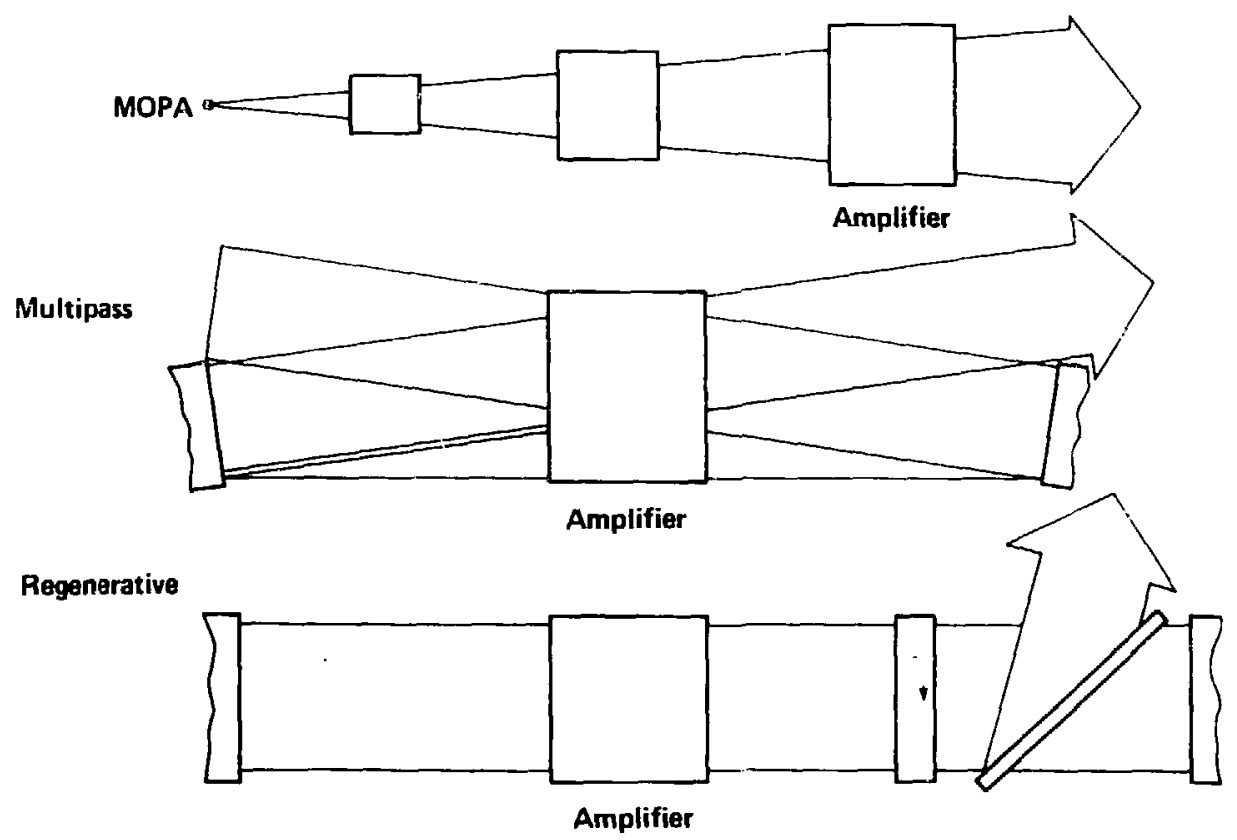

FIG. 73. Siorage laser media--system architeclure. 


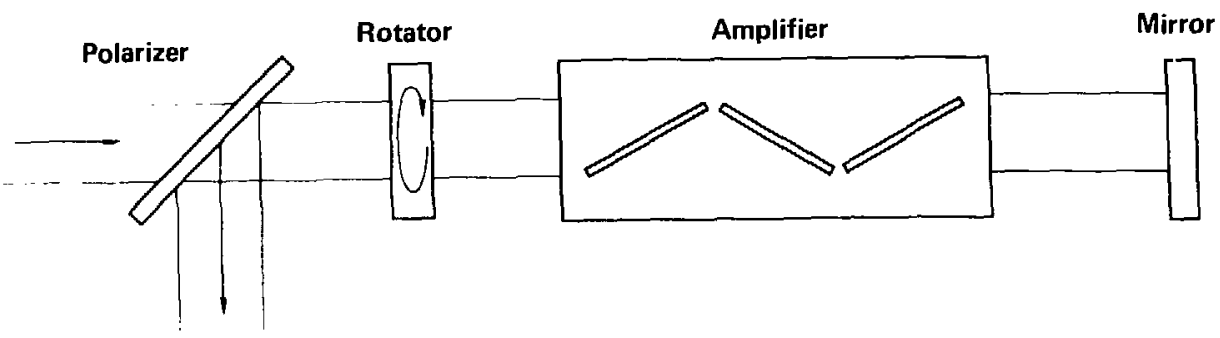

FIG. 74. Two-pass amplifier design used in Nd:glass laser systems.

While the examples in Fig. 73 were chosen to illustrate three basic ideas in laser-system architecture, many innovative hybrid variations have been used. For example, in Fig. 74 it is common to use a Faraday rotator to pass a beam through a glass-laser disk amplifier. ${ }^{144}$ This technique is useful primarily for low-level beams becausc of the damage level associated with the polarizer and sotator at the output section.

Similarly, one can multipass a final-amplifier stage as in Fig. 75 to raise a low-level beam to a fluence level sufficient for an ultimate extraction pass as on the Helios $\mathrm{CO}_{2}$ laser at Los Alamos. ${ }^{140,145}$

Other variations have been proposed and tested. Difficultics have arisen with schemes similar to these above because of fluence limitations, nonlinear aberrations, and parasitus oscillation limitations. To be effective, these multiple-use schemes must sutisfy the same constraints as any other system.

\section{SYSTEM DESIGN}

A common thread in all laser-system designs is the idea of transfer ring energy from stored atomicinversion energy to electromagnetic beam energy in a performance-optimum fashion. ${ }^{146}$ This transfer of energy accurs efficiently when the beam fluence exceeds the saturation fluence of the laser medium. For example. saturated fluences are $4-5 \mathrm{~J} \mathrm{~cm}^{2}$ in a $1-n s$ pulse in $N d: g l a s s$ laser or $0.11 / \mathrm{cm}^{2}$ at 1 ns in a $1-a t m \mathrm{CO}_{2} \mathrm{am}$ plifier. In addition. the laser beam should propagate at a fleence near a propagation limit usually defined by

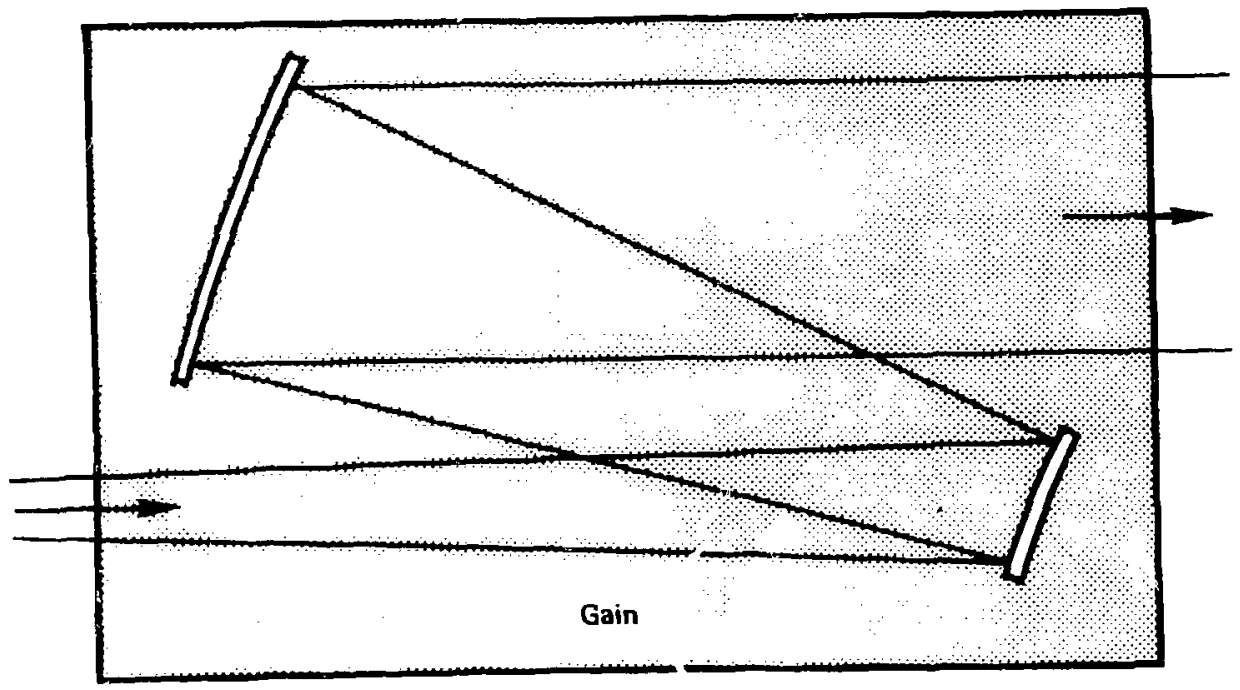

FIG; 75. Three-pass amplifier design used in the Helios $\mathrm{CO}_{2}$ laser system. 

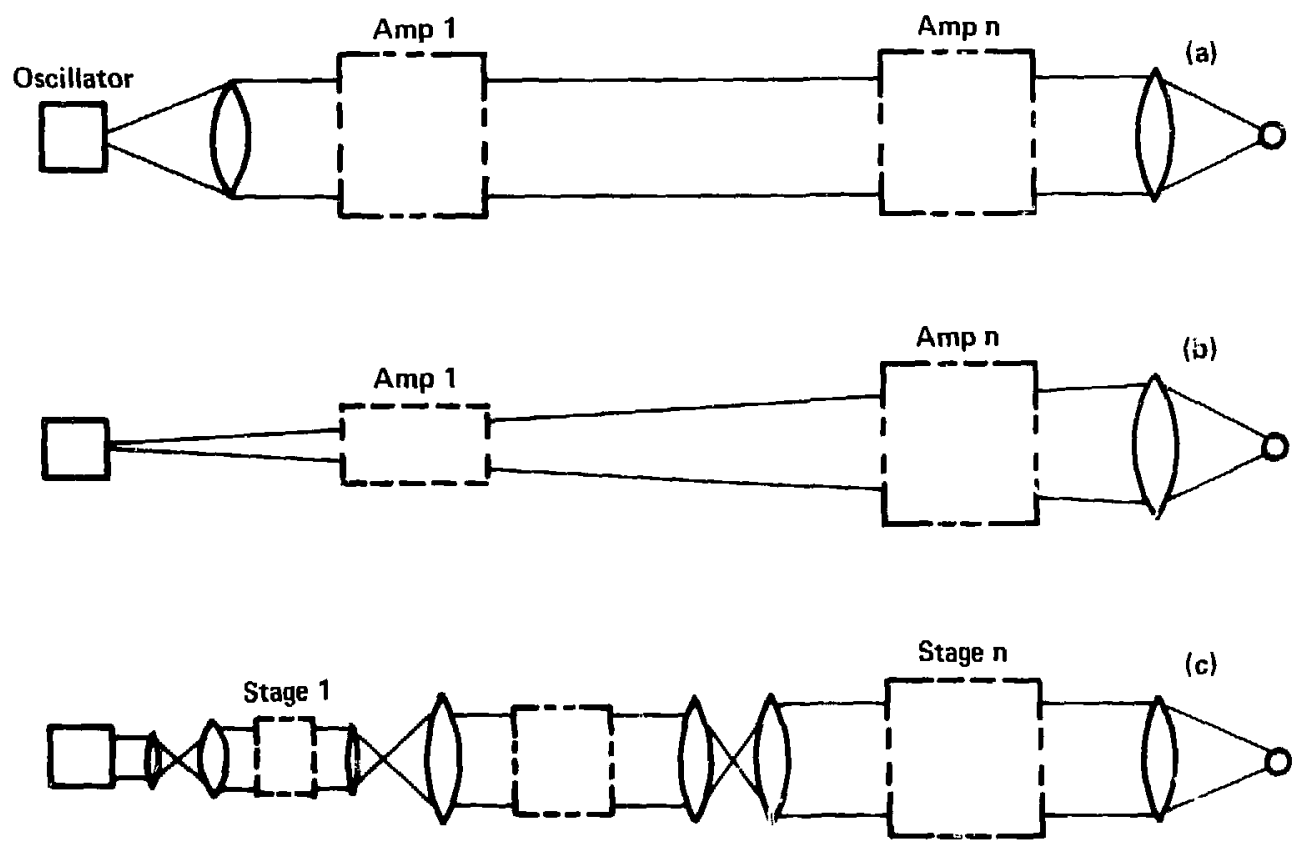

FIG. 76. Examples of system design, showing how amplifier size and beam expansion infucnce system costs.

dielectric breakdown $\left(3-15 \mathrm{~J} / \mathrm{cm}^{2}\right)$, or by nonlinear effects such as self-focusing or two-photon absorption. Unless the system is operated at or above a saturation fluence. energy stored in the amplifier medium is left unused and, unless operation occurs near a breakdown fluence. optical area is purchased that is not required. In addition to these laser fluence considerations. cumulative linear and nonlinear propagation effects will also constrain system performance. In summary, the following considerations determine system operating levels.

- Damage limits.

- Accumulation of nonlinear phase distortion.

- Accumulation of linear phase distortion.

- Amplifier energy storage (gain) vs system loss.

As examples of these considerations in system designs, consider the systems in Fig. 76. The purpose of each system is to amplify the oscillator palse from the $\sim 1-\mathrm{mJ}$ oscillator output to a fuence limit imposed by the exit aperture area and the length of propagation. However, system (a) in Fig. 76 is too expensive. Amplifier $I$ is not used efficientiy: the laser intensity is well below the saturation fluence and propagation limits: thus little of the (expensive) stored energy is extracted. In addition the gain of a larger-diameter amplifier is usually lower than the gain in smaller-diameter amplifiers like those used in the early stages of (b) and (c). Thus additional amplifiers are required, imposing larger propagation distances through gain media and accumulatung additional beam aberrations. Concept (a) however, could be used in multipass systems (e.g.., the multipass system in Fig. 73, where amplifier I replaces amplifier 2 to $\mathrm{n}$, and the low extraction efficiency per pass is overcome by reusing the amplifier many times. When propagation constraints are satisfied, this technique is thought to be efticient.

System (b) in Fig. 76 is a conceptually simple approach to matching the rate of increase in laserbeam intensity to the increase in amplifier diameter. The rate of divergence of system (b) is set by the amplifier gain and the accumulation of aberrations. A rapid divergence is associated with high-gain amplifiers (nonsaturated) and short path length to limit nonlinear aberration accumulations: a lenger, more gentle taper is 
associated with saturated gain and more gradual accumulation of nonlinear aberrations. The system, as it is portrayed in Fig. 76 (b) with a continuously expanding beam, does not provide for image relaying.

System (c) in Fig. 76 is the arrangement for present Nd:glass laser systems. The growth of the beam diameter is optimized to extract energy from the amplifier as efficiently as possible and to stay within damage limits and nonlinear-aberration limits. The use of expanding telescopes to increase beam diameter also permits relay imaging and spatial filtering to be done conveniently. Each stage contains the necessary components for isolation, amplification, and imaging and meets nonlinear and linear propagation constraints.

\section{SUMMARY OF SOME RULES FOR LASER-STAGE DESIGN}

tion.

- The gain length within a given amplifier stage must be such that there is no internal stage oscilla-

- Linear and nonlinear aberration constraints are satisfied and damage limits are not exceeded.

- The amplifier diameter is selected to amplify the low-level oscillator signal as cheaply as possible to an output level determined by the propagation limitations associated with both the output aperture and the cumulative aberration limits.

\section{STAGING}

Laser chains are conveniently divided into stages. The system in Fig. 76 (c) is examined in more detail as it is a common design and the ideas are easily applied to similar chain configurations. Figure 77 shows a stage of this chain. This stage consist: of an input element, in this case a lens with a coating (1); an amplifier stage with gain; a loss element, which includes losses and aberrations on windows. disks, Faraday rotators, lenses, etc.: and an exit element, in this case coated or uncoated lenses (2).

Because of the variation in component performance with laser-pulse duration. the stages. and consequently the laser system. can be optimized only at one pulse duration. The performance of the system at another pulse duration, while adequate, will be less than optimum because the system is not optimized at that specified pulse duration. Three clear regimes of system design occur, limited by the above constraints. The short-pulse operation region $(<50 \mathrm{ps})$ is where nonlinear-aberration constraints are dominan, the intermediate-operation regime is where surface-damage constraints limit performance, and the long-pulse operation regime is where the laser-gain (stored energy)-stage-loss ratio determines performance. Examples of lasers in each regime are short-pulse ( $50 \mathrm{ps)} \mathrm{Nd:glass} \mathrm{laser} \mathrm{systems,} \mathrm{which} \mathrm{are} \mathrm{nonlinear-aberration}$ dominated, the $\mathrm{CO}_{2}$ laser system operating at $\sim 1 \mathrm{~ns}$, which is damage limited, and the $\mathrm{KrF}$ system at $\sim 10 \mathrm{~ns}$. which is stored-energy limited. Figure 78 shows the regimes of operation for Nd:glass lasers.

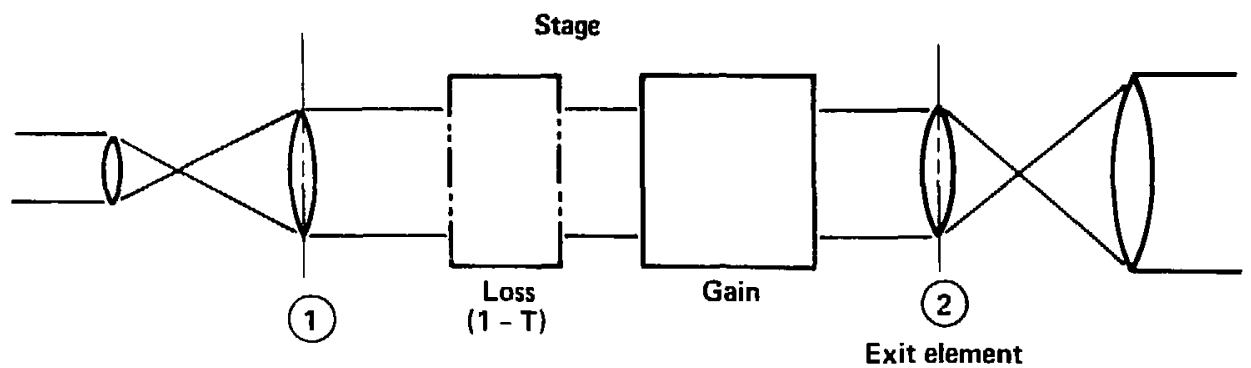

FIG. 77. A typical stage in a laser chain. 


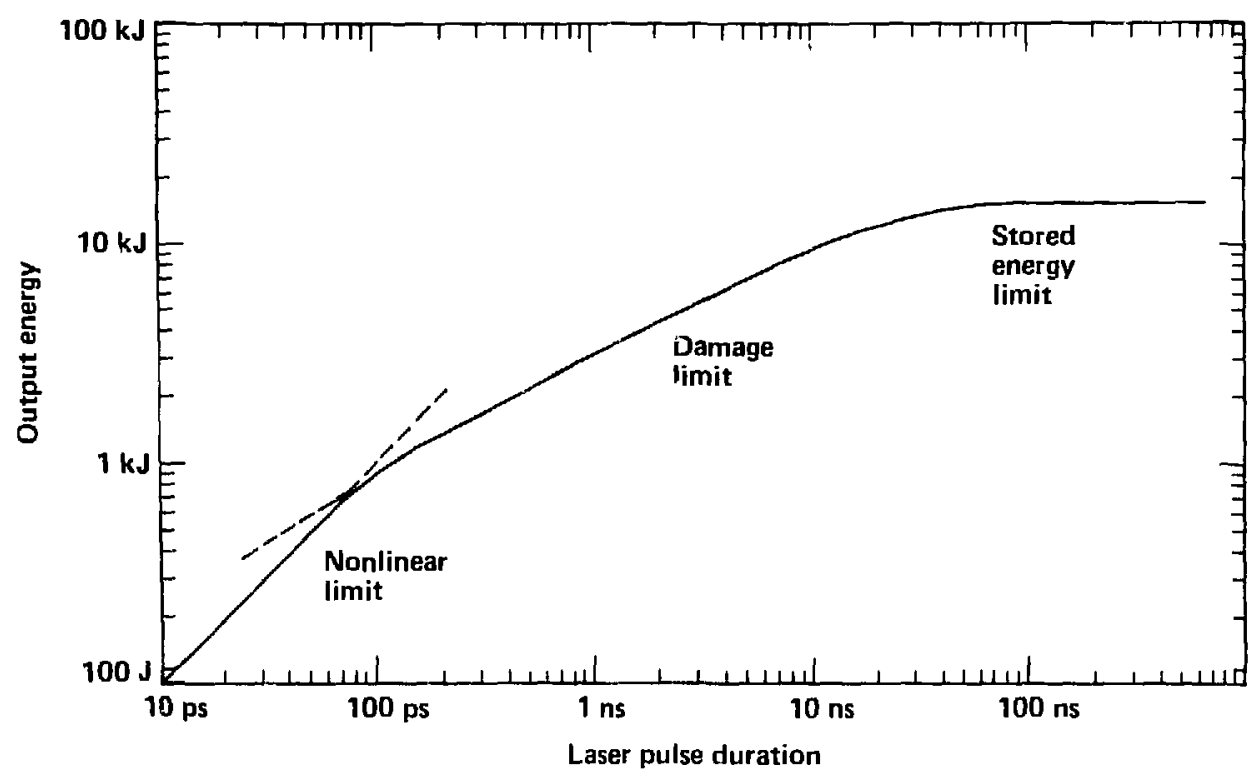

FIG. 78. A laser chain is constrained in its optical output by different phenomena at different pulse durations.

Laser-system designs are optimized at a singie-pulse duration. For a Nd:glass system, the dominant limiting mechanisms are shown. Other systems are constrained by similar limits. The constraints are summarized below:

I. Nonlinear aberrations limit short-pulse performance:

$B=\frac{2 \pi}{\lambda} \int_{\text {input }}^{\text {output }} \gamma ! \mathrm{d} \ell<$ constraint $\sim 4$ rad of nonlinear retardation between spatial filters

In this case nonlinear aberrations accumulate rapidly and deteriorate beam focusability before damage limits are reached. With ultraclean relayed systems this constraint may be relaxed somewhat to $B \approx 4$.

2. Damage levels of the output aperture limit the total laser fluence:

$\mathscr{E}_{\text {Liset }} \leqslant \mathscr{E}_{\text {dimage }}$.

3. Laser-beam energy is limited by the amplifier stored energy and the stage losses.

$E_{\text {buan }} \leqslant \frac{\alpha}{\gamma} E_{\text {sat }}$.

To optimize stages for each performance region, the components are first optimized. ${ }^{146}$ In particular, a great deal of attention is paid to amplifier performance, since it is the most costly element and it contributes a major fraction of the nonlinear and linear aberrations.

Under constraint 1) above, the amplifier is optimized so that gain per unit contribution of nonlinear aberration (B integral) is maximum. In glassndisk amplifiers this means that thin, strongly pumped laser disks are selected (see Fig. 79). Amplifier electrical efficiency is sacrificed to maximize fluence through the whole stage. At the same time, the thickness of the other optical components is reduced to minimize nonlinear phase accumulation. With amplifiers so optimized, the best siluation possible for chain construction is obtained. These stages are then assembled to make a laser system. ${ }^{15.79}$ Nonlinear-aberration constraints of <4 rad/stage (including the focusing stage) are usual, with overall-chain ( $\Sigma$ stages) cumulative nonlinear aberrations of 


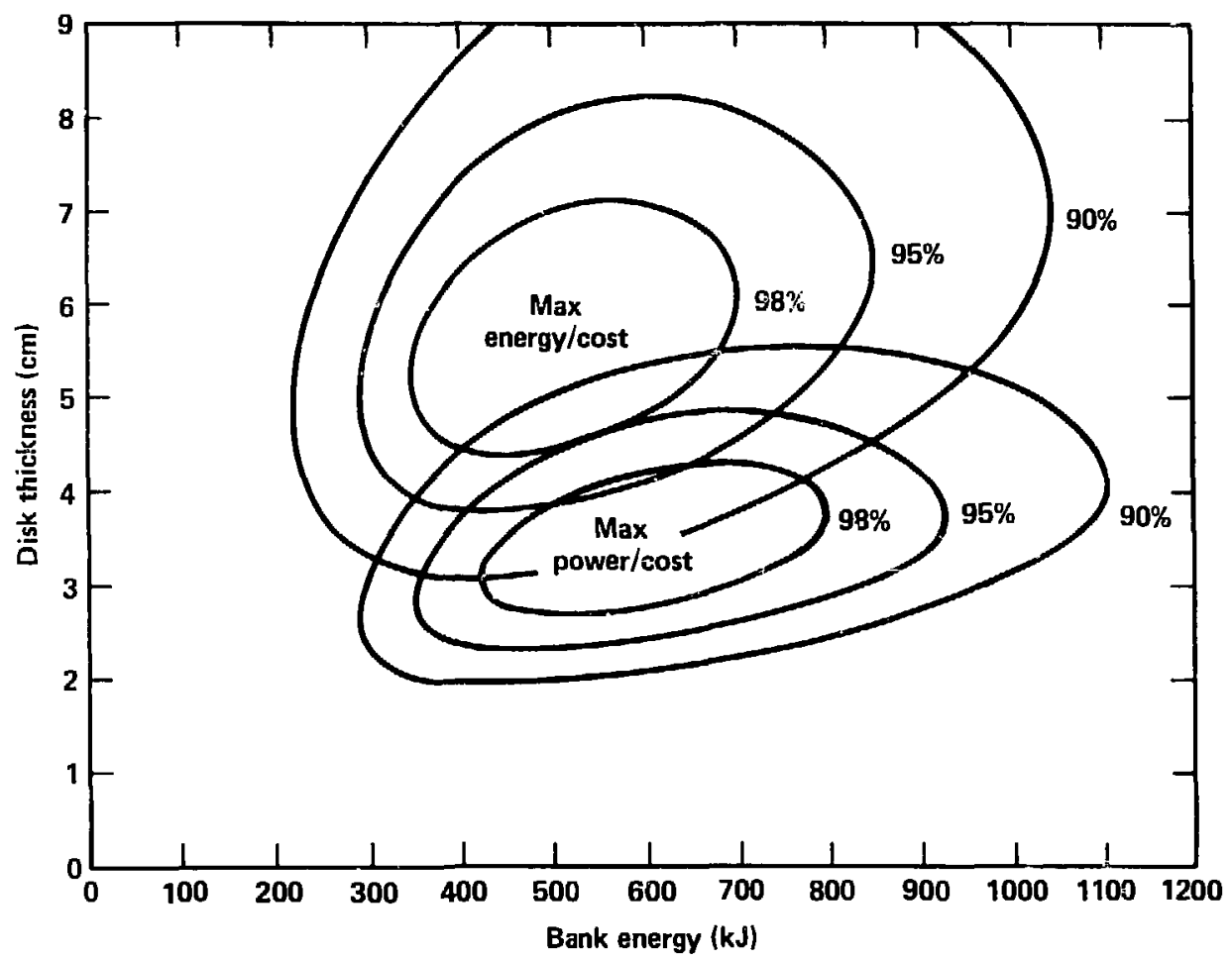

FIG. 79. Nd:glass amplifier optimization by varying bank energy and disk thickness. Maxima for energy occur with thicker glass disks than the maxima for power performance.

$\sim 10$ rad. Such a system optimization emphasizes small-diameter, high-gain amplifiers, thin optical materials, and short systems. See Fig. 80, for example, which shows the Argus staging consideration. The optimization of a system using chains constructed from these stages requires the consideration of the additional costs associated with each chain. These costs include chain diagnostics, automatic pointing, control, etc. When these considerations are included, it is found that larger-diameter chains are desired to minimize the number of chains and thus, total costs.

In a system where nonlinear (and linear) phase accumulation is not an issue, the design is governed by the efficiency of energy storage in the amplifier. In this case, the efficiency of the amplifier is optimized becauss with the constraint of either a damage limit or stored energy limit leads to the lowest-cost stage. Figure 79 also shows an example of glass laser disks in an amplifier optimized for long-pulse operation.

The total gain in the stage is determined either by the stage losses, since there is an optimum gain-toloss ratio, or in present cascaded systems by the ratio of the damage threshold of the entrance optic to the exit optic. With present damage thresholds, the optimum technique for long-pulse system synthesis is shown in Fig. 81 .

This technique, used to optimize performance in long pulse Nd:glass laser systems, is to let the exit fluence of an amplifier approach the damage threshold of uncoated optics. ${ }^{147}$ The area expansion ratio of the telescope is the ratio of damage thresholds, modified by the attenuation in the peak noise due to the spatialfilter pinhole and the loss term in the first lens. Typical ratios are $\mathscr{E}_{\text {bare }} / \mathscr{E}_{\text {coated }} \sim 2$. The amplifier in tire stage is optimized for energy storage to overcome the stage losses associated with the reflections from the uncoated surfaces at $O_{\text {exit }}^{n}$ and to bring the beam up to the permissible fluence on element $O_{\text {exit }}^{n}$ in Fig. 81 . 


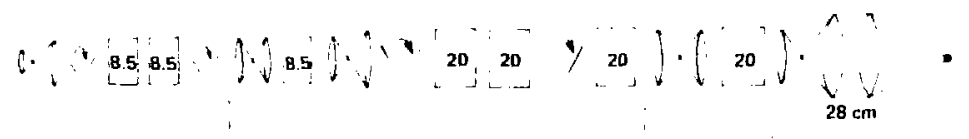

100 ps (2.5 TW)

$\begin{array}{lcccr}\text { Estimated peak flux }\left(\mathrm{J} / \mathrm{cm}^{2}\right) & 0.3 & 1.1 & 1.28 & 1.87 \\ \text { Break-up integral "B" } & 0.55 & 1.9 & 4.50 & 6.69 \\ \text { Beam energy (J) } & 11 & 33 & 162 & 268\end{array}$

\section{1 ns (1 TW)}

Estimated peak flux $\left(\mathrm{J} / \mathrm{cm}^{2}\right)$
Break-up integral " $\mathrm{B}$ "

Beam energy (J)

$$
\begin{aligned}
& 3.4 \\
& 0.93
\end{aligned}
$$

60
5.2

1.81

175
3.7

2.97

714
5.2

3.79 1040

FIG. 80. Argus staging.

Isofluence means that each stage exit aperture is used to its utmost capability. This minimizes the costs of each stage, which are proportional to the aperture area.

In summary, the steps required to optimize a laser stage are to select the laser-pulse duration and to use amplifiers optimized for the pulse regime. If the operating regime is not clear cut, that is, short-pulse nonlinear limited or long-pulse fluence limited, or if an extremely versatile laser system is needed, amplifier parameters halfway between short-pulse and long-pulse opimized devices are selected (Fig. 79). The next step is to use optimized amplifiers to build up chain stages, subject to constraints of stage construction. These are nonlinear phase accumulations, isolation requirements, and fluence limitations. Once an optimized stage is obtained (for each diameter), the stages can be assembled into a chain, and the chains assembled into a system. This process may have to be iterated several times.

An example of a short-pulse optimized Nd:glass-laser chain is the Argus laser chain ${ }^{15}$ shown in Figs. 80 and 82. The Argus chain is nonlinear-phase-optimized (B limited) for 100-ps laser pulses. Under these conditions the nonlinear phase retardation per stage is balanced, the system is isolated against $100 \%$ target back reflection, and the laser fluence never exceeds commercial thin film damage limits. Figure 9 shows the focal characteristics of this system. The output section of the system was recently expanded to improve the long-pulse performance. Additional $1.4 \times$ magnification from 20 to $28 \mathrm{~cm}$ at the amplifier exit permitted the amplifier stage to amplify the beam to greater intensity than the focusing lens could tolerate when it was $20 \mathrm{~cm}$ diameter, thus improving the long-pulse energy performance. The University of Rochester's Omega laser system has been designed using similar rules. ${ }^{139}$

Another example of a laser chain is the Nova laser system shown in Fig. 83. This particular chain is optimized to be efficient from $100 \mathrm{ps}$ to $2 \mathrm{~ns}$. The use of a low nonlinear-index-of-refraction fluorophosphate laser glass has permitted very efricient amplifier operation within nonlinear-aberration constraints. The system is designed to become isofluent at $\sim 2 \mathrm{~ns}$, where the fluence limits permit an intense-enough laser beam to obtain good energy extraction from the amplifier. The use of uncoated lenses at the exit of each stage permits average beam intensities to approach $10 \mathrm{~J} / \mathrm{cm}^{2}$ at $1 \mathrm{~ns}$, much higher than the $5-7 \mathrm{~J} / \mathrm{cm}^{2}$ damage limit of antireflection coatings. 


\section{Stage n}

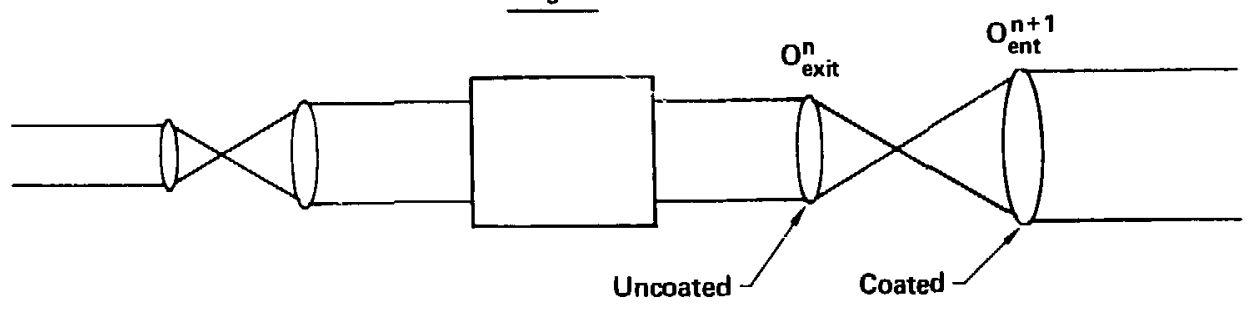

FIG. 81. Expansion technique used in damage-limited long-pulse Nd:glass laser systams.

\section{SYSTEM COSTS}

The costs of a large laser system can be divided into approximately four equal eategories ${ }^{148}$ :

- Optics.

- Eletrical equipment.

- Mechanical equipment.

- Buildings and contingeney.

In this report the optical performanee of a laser chain has been emphasized, since a great deal of leveruge is available in this area. Any increase in the fluence-handling eapucity of an aperture leads to a direct

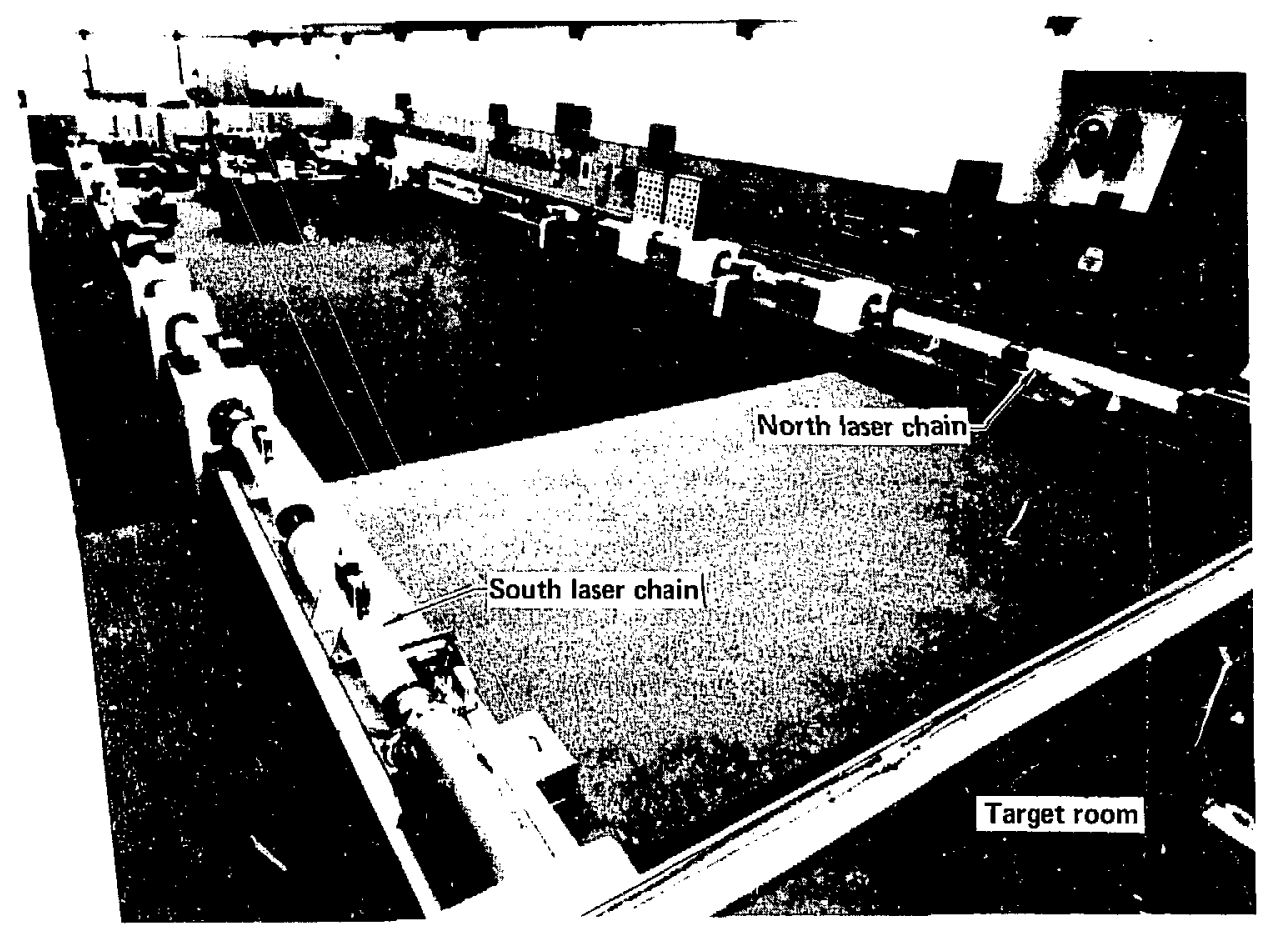

FIC. 82. Argus laser irradiation facility. 


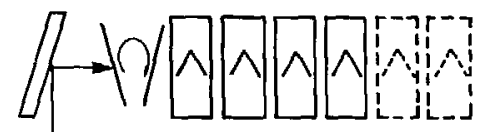

$3: 5 \mathrm{~cm}$ disk amps

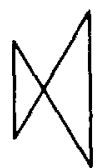

$46 \mathrm{~cm}$ disk amps

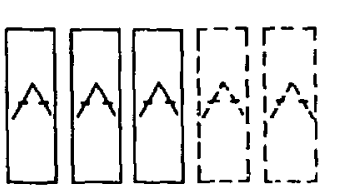

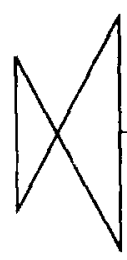

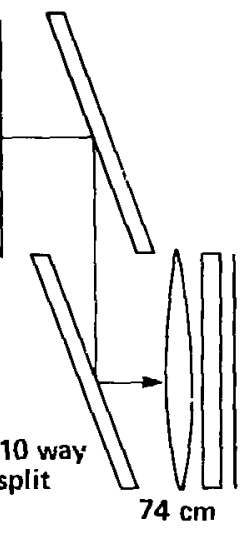

$74 \mathrm{~cm}$

$\mathbb{N}$

\subsection{0 .820 .8}
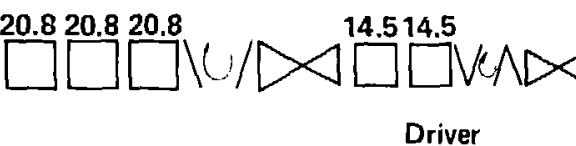

9.4

9.4
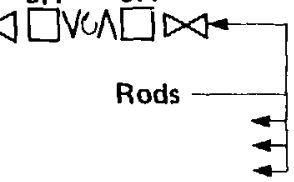

^ $\mathrm{N}-\mathrm{cm}$ aperture amplifieis

\section{$\infty$ Spatial filters}

IV Faraday isolators

8 Mirrors

에 Focus lens, window, debris shield

FIG. 83. Nova chain design.

increase in system performance with little cost increase. For example, the implementations of spatial filters and relay imaging have each led to significant increases in the fluence capacity of laser operations for a negligible cost increase. Also, the isofluence concept used on Nova will permit a $2 X$ increase in aperture fluence, with a small chain-cost increase associated with extra gain to overcome the $7 \%$ loss of the uncouted exit optic. Cunversely, a mistake in the physics or engineering of the optical system can lead to an enormous reduction in laser performance. Past examples have been the lack of attention to chain-target isolation, inaccurate assessments of nonlinear aberrations limits (B limits), and overly optimistic estimations of laser-optics damage thresholds.

The designs of the electrical and mechanical subsystems strongly influence the ease with which the systems can be assembled, operated, and maintained. Performance or cost improvements in these areas do not influence the total system performance dramatically as do optical-system improvements. Nonetheless, constant improvement is being made in these areas. Figure 84 shows the improvements in Nd:giass.laser-system performance with innovation and with improved cost engineering.

Experience also shows that the capital cost of a laser chain is distributed as shown in Fig. 85. As a result, a great deal of attention is paid to engineering the amplifier section, which includes isolators and spatial filters, as well as amplifiers. Since the amplifier costs scale with area, the division of the total required beam area into smaller subsections (more chains) is permitted with small cust penalties on amplifier performance. However, the diagnostics, control, and maintenance costs are very nearly the same for each chain no maiter what the chain diameter. As a consequence, the use of as small a number of chains as possible is emphasized, each with as large a beam area as possible. As an example of this at Livermore, the Janus laser used an $8.5-\mathrm{cm}$ diam beam, Argus and Shiva a 20 -cm-diam beam, an early Nova design a $50-\mathrm{cm}$-diam beam, arid the present Nova design has a $74-\mathrm{cm}-$ diam output beam. 


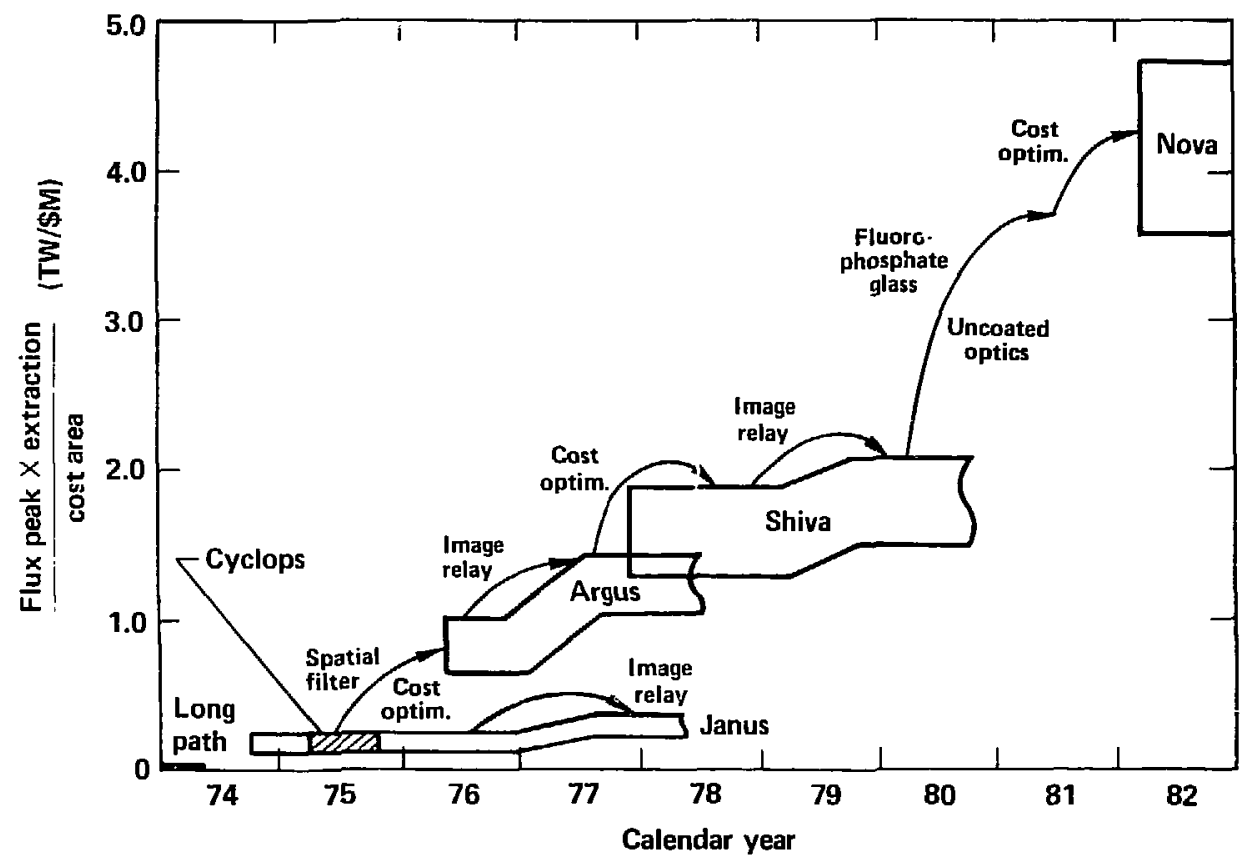

FIG. 84. Performance/cost breakthroughs on solid-state laser systems.

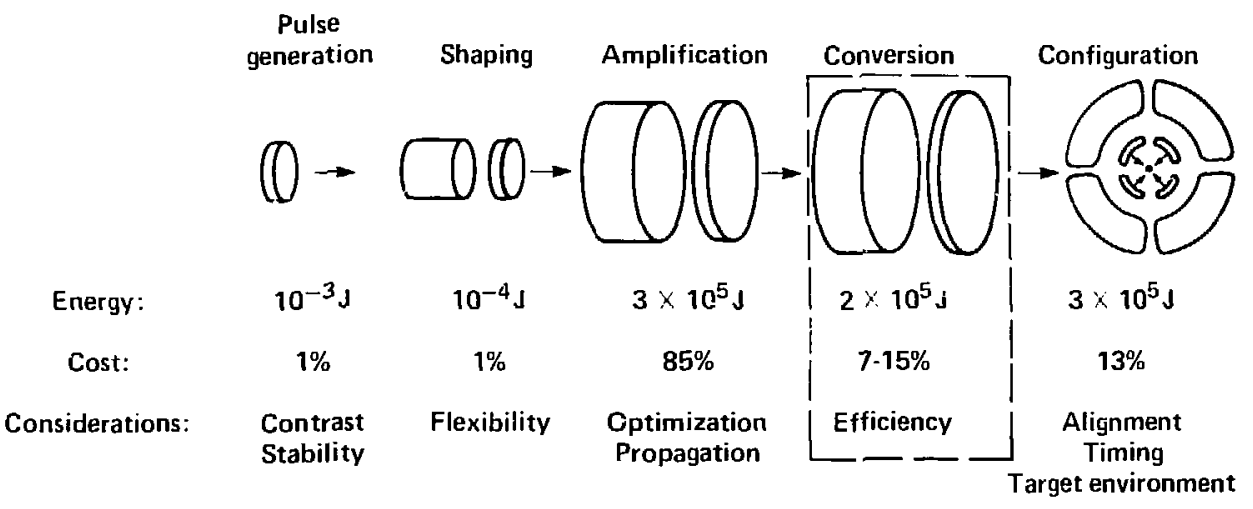

FIG. 85. Fusion laser functions and costs.

\section{THE FUTURE}

Futuize laser systems for fusion application will be required to deposit $\sim 1-5 \mathrm{MJ}$ of energy on target in 10-20 ns pulse durations. The target irradiation rate must occur at $1-10 \mathrm{~Hz}$. Such laser systems will undoubtedly be of the multipass architecture and will possess $\sim 1-3 \mathrm{~m}$ diam apertures. ${ }^{149}$ Gas-laser systems are favored because of the simplicity of cooling and potential high efficiency. These systems are technically feasible, but require additional work on materials to permit the highest possible fluence through the laser apertures to maintain reasonable overall system costs. The constant increase in performance per dollar, shown in Fig. 84, should continue as laser technology evolves. 


\section{REFERENCES}

1. S. Colgate, R. E. Kidder, J. H. Nuckolls, R. F. Z :bawski, and E. Teller, Lawrence Livermore Laboratory, Livermore, $\mathrm{CA}_{2}$ unpublished calculations (1951).

2. N. G. Basov and O. N. Krokhin, "The Conditions of Plasma Ficating by the Optical Generator Radiation," in Proc. 3rd Quantum Electronics Conference, Paris. 1963, N. Bloembergen and P. Grivet, Eds. (Columbia University Press, NY, 1964).

3. J. H. Nuckolls, L. L. Wood, A. R. Thiessen, and G. B. Zimmerman, "Laser Compression of Matter to Super-High Densities: Thermonuclear (CTR) Applications." Nature 239 (5368), 139-192 (1972).

4. K. A. Brueckner and S. Jorna, "Laser Driven Fusion," Rev. Mod. Phis. 46, 325 (1974).

5. J. L. Emmett, J. H. Nuckolls, and L. L. Wood, "Fusion Power by Litier Implosion," $S_{c i}$. Am. pp. 24-37 (June 1974).

6. G. Charatis, J. Downard, R. Goforth, B. Guscat, T. Henderson, S. Hildum, R. Johnson. K. Moncur, T. Leonard, F. Mayer, S. Segal, L. Siebest, D. Solomon, and C. Thomas, "Experimental Study of" Laser Driven Compression of Spherical Glass Shells," in Proc. of the 5 th IAEA Plasma Fusion Conf. in" Tokyo, Japan, 1974 (Plasma Physics and Controlled Nuclear Fusion Research 1974, 2, 317 IAEA. Vienna, 1975).

7. J. F. Holzrichter, H. G. Ahlstrom, R. D. Speck, E. K. Storm, J. E. Swain, L. W. Coleman, C. D. Hendricks, H. N. Kornblum, F. Sewaıd, V. W. Slivinsky, Y. L. Pan, G. B. Zimmerman, and J. H. Nuckolls, "Implosion Experiments with an Asymmetrically Irradiated Laser Fusion Target," Plasma Phys. 18, 675-680 (1976); K. R. Manes, H. G. Ahlstrom, R. A. Haas, and J. F. Holzrichter, "LightPlasma Interaction Studies with High-Power Glass Lasers," J. Opt. Soc. Am. 67, 717 (1977).

8. Laser implosion experiments are being carried out by workers at Los Alamos Scientific Laboratorits. Los Alamos, NM; Rutherford Laboratories, England; Naval Research Laboratories, Washington. D.C.: University of Rochester, Rochester, NY; CEA in Limeil, France; The Lebedev Institute. Moscow, USSR; and Osaka University, Japan.

9. A. L. Schawlow and C. H. Townes, "Infrared and Optical Masers," Phys. Rev. 112 (6), 1940-1949 (1958).

10. D. Ross, Lasers, Light Ámplifiers, and Oscillators (Academic Press, NY, 1968).

11. Laser Theory. F. Barnes, Ed. (IEEE Press, NY, 1972).

12. Laser Interaction and Related Plasma Phenomena, Vols. 1-4, H. J. Schwartz and H. Hora, Eds. (Plenum Press, NY, 1971-77).

13. Laser Program Annual Reports 1973-1978, Lawrence Livermore Laboratory, Livermore, CA, UCRL$50021-73,-74,-75,-76,-77,-78$. These reports are avalzble from the National Technical Information Service, Sprıngfield, VA 22161.

14. W. F. Krupke, E. V. George, and R. A. Haas, "Advanced Lasers for Fusion," in The Laser Handbook. M. L. Stitch, Ed. (North Holland Publ. Co., 1979), p. 627.

15. W. W. Simmons, D. R. Speck, and J. T. Hunt, "Argus Laser System: Performance Summary," Appl. Opt. 17, 999 (1978).

16. A. E. Siegman, An Introduction to Lasers and Masers (McGraw-Hill Book Co., NY, 1971).

17. A. Yariv, Quantum Electronics (John Wiley \& Sons, NY, 1967).

18. E. Snitzer, "Optical Maser Action of $\mathrm{Nd}^{3+}$ in a Barium Crown Glass," Phys. Rev, Lett. 7, 444 (1961).

19. E. Snitzer, "Glass Lasers," Appl. Opt. 5, 1487 (1966).

20. W. F. Krupke, "Induced-emission Cross Sections in Neodymium Laser Glasses," IEEE J. Quant. Elect. QE-10, 450 (1974).

21. S. E. Stokowski, R. A. Saroyan, and M. J. Weber, Nd-Doped Laser Glass Spectroscopic and Phy'sical Properties, Lawrence Livermore Labutatory, Livermore, CA, Misc. Reporl MS-095 (1978).

22. C. K. N. Patel, "Selective Excitation Through Vibrational Energy Transfer and Optical Maser Action $\mathrm{N}_{2}-\mathrm{CO}_{2}$," Phys. Rev. Lett. 13, 617 (19;; ; ;

23. For a complete review of the $\mathrm{CO}_{2}$ literat:"s un to 1974 the reader should consult: $\mathrm{O}, \mathrm{P}$. Wood, "High Pressure Pulsed Molecular Lasets," Proc. IEEE 62, 355 (1974). 
24. See articles in: Principles of Laser Plasmax. G. Bekefi. Ed. (John Wiley \& Sons, NY, 1976).

25. K. Hohla and K. L. Kompa, "Photochemical lodine Laser - A High Power Gas Laser," in Laser Interaction and Reliated Plasma Phenomena. Vol. II. H. J. Schwarty and H. Hora. Eds. (Plenum Press. NY, 1971), p. 61.

26. N. G. Basov et al., Kvant. Elektron 6, 116 (1973).

27. K. Hohla and K. L. Kompa, "The Photochemical lodine Laser," in Handbook of Chemical Lasers. R. W. F. Grose and J. F. Batt, Eds. (John Wiley \& Sons, NY, 1976), p. 667.

28. G. Brederlow, K. J. Witte, E. Fill. K. Hohla, and R. Volk, "The Asterix III Pulsed High-Power lodine Laser," IEEE J. Quant. Elect. QE-12, 152 (1976).

29. G. A. Fisk, M. A. Gusinow, A. K. Heys, T. D. Padrick, R. E. Palmer, J. K. Rice, M. E. Riley, and F. K. Truby, The Atomic lodine Laser, Sandia Laboratories, Albuquerque, NM, Technical Report SAND 78-1071 (1978), available from National Technical Information Services, Springlield, VA. 22161 .

30. J. J. Ewing and C. A. Brau, "Laser Action on the $2_{1}$ I $2_{1}$. Bands of KrF and XeCl," Appl Phy!s. Lett. 27, 350 (1975).

31. J. A. Mangano and J. H. Jacob, "Electron-Beam-Controlled Discharge Pumping of the KrF Laser," Appl. Phys. Lett. 27, 495 (1975).

32. For an up-to-date review on the subject of excimer lasers see IEEE J. Quant. Elect. QE-15 5 (1979).

33. L. M. Frantz and J. S. Nodvik, "Theory of Pulse Propagation in a Laser Amplifier." J. Appl. Phys. 14. 2346 (1963).

34. N. G. Basov and V. S. Letckov, "Propagation of a Light Pulse in an Inversely Populated Medium," Opt. Spektrosk. 6, 586 (1965).

35. J. B. Trenholme and K. R. Manes, A Simple Approach to Laser Amplifiers, Laurence Livermore Laboratory, Livermore, CA, UCRL-51413 (1972). The deriviation of the saturated gain equations in the lecture notes are due to J. B. Trenholme.

36. For a clear discussion of Fourier optics see: J. W. Goodman, Introduction to Fourier Optics (McGrauHill Book Co., Inc., NY, 1968).

37. For a complete discussion of diffruction, focusing. aberrations, etc., set: M. Born and E. Wolf. Principles of Optics (Pergamon Press, NY, 1964).

38. There is an extensive bibliography on lens design that the reader should consult. The reference given is one example of lens design for last-fusion applications. A. Greenbaum, A. J Glass, and J. B. Trenholme, "Lens and Mirror Design via the Principal Surface," Appl. Opt. 15, 2579 (1976).

39. Figures 26 and 27 were kindly' supplied by Itek Corporation, Lexington, MA.

40. P. A. Renard and J. T. Hunt, ARTEMIS-A Diffrartion Model for Laser Light Propagation. Lawrence Livermore Laboratory, Livermore, CA, UCID-17381 (1977). A. J. Glass and W E. Warren. Malaprop-List Propagation in a Nonlinear Medium with Stochasiic Perturbations, Lawrence Livermore Laboratory, Livermore, CA, UCRL-80316 (1977).

41. N. H. Burnett and M. C. Richardson, "Sparkgap Optical Isolator for High Power Infrared Lasers," Rev. Sci. Instrum. 47, 241 (1976).

42. R. F. Benjamin, D. B. Henderson, K. B. Mitchell, and M. A. Stroscio, "Laser-Target Retropulse Isolation Using an Aperture in Vacuum," Appl. Phys. Lett. 31, 511 (1977).

43 L. P. Bradley and P. Koert, Plasma Shater for High Power Glass Laser, Lawrence Livermore 1.aboratory, Livermore, CA, UCR L-81364 (1978).

44. M. A. Summers, B. M. Carder, J. Myall, W. W. Simmons, and D. R. Speck, Laser-Target and Laser Isolation for Shiva, Lawrence Livermore Laboratory, Livermore, CA, UCRL-80194 (1978).

45. J. B. Trenholme, Fluorescence Amplification and Parasitic Oscillation Limitations in Disk Lasers, Nav i Research Laboratory Mismorandum Report 2480 (July 1972).

46. A. N. Chester, "Gain Thresholds for Diffuse Parasitic Laser Modes," Appl. Opt. 12, 2139 (1973).

47. J. A. Glaze, S. Guch, and 3. B. Trenholme, "Parasitic Suppression in Large Aperture Nd:Glass Disk Laser Amplifiers," Appl. OY!t. 13, 2808 (1974). 
46. J. M. McMahon. J. L. Emmeli, J. F. Holarichter. and J. B. Trenholme, "A Glass Disk-Laser Amplifier." IEEE J. Quant. Elect. QE-9. 992 (1973).

14 For a descussion of amplified spontancous cmession in Nd:glass lasers, see Laser Program Annual Report. Lawrence Livermore Laburatory, Livermort. CA. UCRL-5002i-75 (1976). p. 249.

5) D. R. Speck, Laser Program thumal Repurt. Law rence Livermure Laboratory, Livermore. CA. UCRL. S(1021.78 (1979). p. 2-15

\$1. Huorophosphate Laser Glass LG-812, Informatuon Bulleton 7513, Schot Optical Glass, Inc., Duryea. PA (1978).

52 Vew Houa Gilusen for High-Power Laner Systems, Hoya Gilass Campans. Menlo Park, CA (1977)

$\therefore$ ED-2 Silicale Laser Glass. Onens-llinoss (14)79).

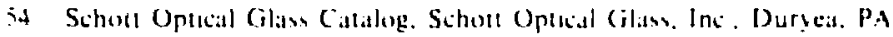

is Ohara Optcal Cilass Cataleg. Buans Opheal Giass, Inc.

in Hoya Opilail Glass Catalog. Hoya Glass Wurk, Menlo Park. CA (1975).

57. Hoya Faraday Rolator Gilass. Husa (jlatse Works, Menlo Parh, CA (1974).

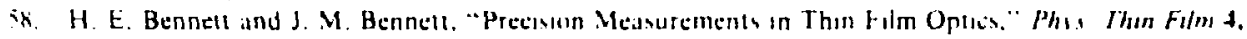
(1967).

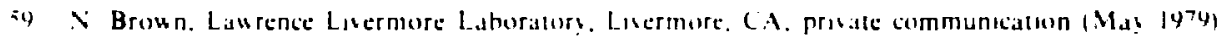

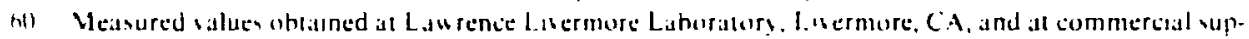
pliers such as Optacal Cowang Lahorators. Ime. Santa Rosa. CA. and Spectra Physats Inc , Mountan Vieu. CA

al. 16. Reichelt, Los Alamos Suentulic Laboratory, Los Alamos, NM. and K. Manes. Lawrence Luermure Lahoratory. Livermort. CA. private sommuntation (June 1979).

0.2 Compendium on High Power Infrared Laser Windaw Materials. Air Force Cambridge Research Center. Cambridge. MA, AFCRC-72-0170 (1972).

n3. H. Mahr, "Two-Pholon Absorplion Spectrosicopy." H. Rabin and C. L. Tang. Eds., Quantum Eler. ironics. Vol. 1. Part II (Acudemic Press, NY, 1975). pp. 285-361.

64. P. Liu. W. L. Smith. H. Lotem. J. H. Bechtel. N. Bloembergen, and R. S. Adhav, "Absulute TwaPhotun Absurpuon Cofficlents at $355 \mathrm{~nm}$ and $266 \mathrm{~nm}, "$ Phyr. Rev. B 17, 4620-4632 (1978).

65. R. 1. Carman, R. Y. Chiao, and P. L. Kelley, "Observation of Degenerate Stimulated Fuur-Photon interacion and four-Wave Parameiric Amplification," Phir. Rev. Lell. 17, 1281 (1966).

66. V. I. Bespatov and V. I. Talanov. "Filamentary Structure of Light Beams in Nunlinear Liquids," JETP LetI. 3, 307 (1966).

67. A. J. Campillo, S. L. Shapiro, and B. R. Snydam. "Penodic Breakup of Optical Beams due to SelfFocusing." Appl. Phis. Letl. 23, 628 (1973).

68. E. S. Bliss, D. R. Speck, J. F. Holerichter, J. H. Erkkila, and A. J. Glass, "Propagation of a HighIntensity Laser Pulse With Small-Scale Intensity Modulation," Appl. Phys. Letr. 25, 448 (1974).

69. "Small-Scale Instability Growth" and "Whole-Beam Self-Focusing," Laser Program Annual Report, Lawrence Livermore Laboratory, Livermore, CA, UCRL-50021-74 (1975), pp. 179-205.

70. Y. R. Shen, "Self-Focusing: Experimental," and J. H. Marburger, "Self-Focusing: Theory," in Progress in Quanrum Elecironics, Vol, 4. Parl I, J. H. Sanders and S. Stenholm, Eds. (Pergamon Press. NY. 1975).

71. E. S. Bliss, D. R. Speck, and W. W. Simmons, "Direct Interferometric Measurements of the Nonlinear Refractive Index Coefficient $n_{2}$ in Laser Materials," Appl. Phys. Letr. 25, 728 (1974).

72. E. S. Bliss, J. T. Hunt, P. A. Renard, G. E. Sommergren, and H. J. Weaver, "Effects of Nonlinear Propagation on Laser Focusing Properties," IEEE J. Quant. Elect. QE-12, 402 (1976).

73. M. J. Weber, D. Milam, and W. L. Smith, "Nonlinear Refractive Index of Glasses and Crystals," $O p$. Eng. 17, 463-469 (1978).

74. C. S. Wang, "The Stimulated Raman Process," in Quantum Electronics, Vol. I, Part Il, H. Rabin and C. L. Tang, Eds. (Academic Press, NY, 1975), pp. 447-472.

75. C. T. Hseih, N. B. Foltz, and C. W. Cho, "Production of Stimulated Raman Lines in $\mathrm{H}_{2}$ Gas with a Focused Laser Beam," J. Opt. Soc. Am. 64, 202 (1974); and Ref. 74. 
Th S. A Akhmanov. A. I. Kovrygin, and A. P. Sukhorukov. "Optical Harmonic Generation and Optical Frequency Multipliers," in Quantum Electronics. Vol. I. Parl III. H. Rabin and C. L. Tang. Eds. (Academic Press. NY. 1975). pp. 476-586.

$\rightarrow$ J. F. Holarichter and D. R. Speck. "Laser Focusing Limitations from Nonlinear Beam Instabilities," J. Appl. Phys. 47, 2459 (1976).

$\rightarrow$ J. A Glaze, "High Energy Glass Lasers," Opt. Eng. 15, 1.36 (1976).

-4 The derivalion of the far-field growth of small-scalc, self-focusing instabilities has been done by J. B. Trenholme; the only availahle reference is in the Laser Program Annual Report. Lawrence Livermore L.aboralory. Livermore, CA. UCRL-50021-76 (1977). p. 2.337.

.6) W. W. Simmons, S. Guch, Jr., F. Ratner, and J. E Murray. "Terrawatt Laser Performance," IEEE J. Quant Elec QE-11, 31D (1975).

-1 A. Betlinger, C. Charles, J Osmalın, and J. G. Giraud, “Laser Beam Brightness Improvement wath High Power Spatial Filterıng." Opt Commun. 18. 176 (1976).

$\mathrm{K} 2$ An excellent history and review on the subject of laser-materials damage problems is Laser Induced Damage in Optical Materials, 1978, A. J. Glass and A. H. Guenther. Eds. (NBS Special Publication S4l, 1978).

\$1. Also see the revieu article by A. J. Giass and A. H. Guenther, "Material Limitations in Fusion Lasers" in Crivical Materials Prohlems in Energy Production (Academic Press Inc. NY. 1976).

4. These experiments showing the onset of laser damage were performed by D. Milam. W. H. Loudermilk. and F. Raner. The SEM photographs of the damaged samples uere kandly provided by G. Carrier of Cornıng Glass Works, Corning. NY. For details of the experimental techniques, see D. Milam. "Measurement and Identification of Laser-Damage Thresholds in Thin Films," Opt. Coatings-Applications and Utilization If, Soc. Phol. Insinm. Eng. 140, 52 (1978).

85. N. L. Boling. G. Dube, and M. D. Crisp. "Laser Surface Damage Studies on Several Glasses. " in Laser Induced Damage in Optical Materials (NBS Special Publication 387. i973). p. 69: J. H. Apfel. E. A. Enemark. D. Milam, W. L. Smith, and M. J. Weber, "The Effects of Barrier Layers and Surface Smoothness on $150 \mathrm{ps}, 1.06+\mu \mathrm{m}$ Laser Damage," in Laser Induced Damage in Optical Materials (NBS Special Publication 509. 1977), p. 255

86. A review of inclusion-initiated laser damage in optical glass is given in R. W. Hopper and D. R. Uhiman, "Mechanism of Inclusion Damage in Laser Glass," J. Appl. Phys. 41, 4023 (1970).

87. The $100 \mathrm{ps}$ damage thresholds for $0.25 \mu \mathrm{m}$ light were obtained by $T$. Deaton and $W$. L. Smith at the Lawrence Livernore Laboratory, Livermore. CA. The $20 \mathrm{~ns}$ data were obtained by $\mathbf{B}$. W. Newmann and D. H. Gill, "Ultraviolet Damage Resistance of Laser Coatings," in Laser Induced Damage in Optical Materials (NBS Special Publication 541, 1978), p. 100. The $1.0 \mu \mathrm{m}$ dates were obtained by D. Milam, W. H. Lowdermilk, and F. Rainer at the Lawrence Livermore Laboratory, Livermore, CA (1979), and at $10 \mu \mathrm{m}$ by the Los Alamos Scientific Laboratory, Los Alamos, NM.

88. The darnage hresholds for pulse durations of $0.1,1.0$, and 3.0 ns were measured at the Lawrence Livermore Laboratory, Livermore, CA by D. Milam, W. H. Lowdermilk, and F. Rainer. The damage thresholds at $30 \mathrm{~ns}$ were measured by N. Boling, G. Dube, and M. D. Crisp in Laser Induced Damage in Optical Materials, A. J. Glass and A. H. Guenther, Eds. (NBS Special Publication 387, 1974).

89. M. Bass and H. Barrett, "Avalanche Breakdown and the Probabilistic Nature of Laser-Induced Damage," IEEE J. Quan!. Ėlect. QE-8, 338 (1972).

90. W. L. Smith, “Laser-Induced Breakdown in Optical Materials," Opt. Eng. 17, 1353 (1971).

91. J. Maniscalco, J. Blink, R. Buntzen, J. Hovingh, W. Meier, M. Monsler, and P. Walker, Civilian Applications of Laser Fusion, Lawrence Livermore Laboratory, Livermore, CA, UCRL-52349, Rev. 1 (1978).

92. R. M. Feinberg, R. S. Lowder, and O. L. Zappa, Low Pre'ssure Laser Cavity for Repetilively Pulsed Electric Discharge Lasers, Air Force Weapons Laboratory, Kirtland Air Force Base, NM, AFWL-TR$75-99$ (1975).

93. S. N. Vlasov, V. A. Petrischer, and V. I. Talanov, "Averaged Description of Wave Beams in Linear and Nonlinear Media (The Method of Moments)," Izv. Vuz., Radio Fiz. 14, 1353 (1971). 
94. R. A. Haas and A. J. Glass. "Propagation of Laser Energy." Laser Progrant f nmeal Report. Lasurence Livermore Laboratory. Livermore. CA. UCRL.50021.77 (1978). pp. $716-720$.

95. A. J. Glass, "Beam Transport Optus for Laser Fusion," Soc. Phot Insinam. Eng. 121, 111 (1977).

96. P. Jacquinol and B. Roken-Dossicr. "Apodization," Prog. Opt. 3, 31 (1964).

97. B. C. Iohnson, Laser Program Annual Report. Lawrence Livermore Laboratory. Livermerc, ( $A$. UCRL-50021-76 (1977), p. 2-322: UCRL-50021.74 (1975), p. 48.

98. A. J. Campillo. J. E. Pearsun. S. L. Shapiro, and N. S. Terrell. Jr. "Fresnel Diffrateton Effectu in the Design of High-Power Laser Systems." tppl. Phys. Lett. 23, 85 (1973)

99 J. T. Hunt. J. A. Glass, W. X. Simmans, and P. A. Renard, "Suppression of Self-l ocusing Thruph Low-Pass Spatial Filtering and Relas Imagung." Appl $O_{p 1}$ 17, 2053 (1978).

100. The improvements in the performance of the Argus laser systens are given by $J \mathrm{~T}$. Hunt, “Argus lmprovements." Laser Program fnnual Repart, Lawrence Locemare Lahoratory. Lwermore. ( A. UCRL-50021-78 (1979). P. 2-133.

101. M. U. Crisp. N. L. Boling. and G. Dube. "Importance of Fresnel Reflections in Laser Surface Bamitec of Transparent Diclectrics," Appl. Phis. Le'lt 21.364 (19)72).

102. J. B. Trenholme, "The Ghase Focusing Code," Laser Program fnmual Reporr. Lawrencte Lisermuse Laboratory, Lwermure, CA. UCRL -50021.75 (1976), P. 157

103 The heuristic deseription of oscillator modes in thos report follows that of D. Russ. Rel. I0. PP. 4-5

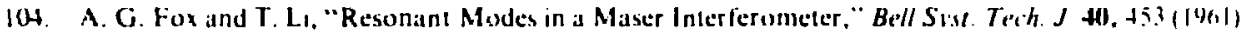

I0\$ H. Kugelnik and T. L1, “Laser Beams and Resonators," Prot. 1GEE 54, 1312 (1966).

106. P. W. Smith, "Mode Selection in Laser," Proc. IEEE 60, 422 (1972).

107. A. E. Siegman, Ref. 15, p. 293.

108. The oscillograms, streak camera pictures, and Fabry-Perot interferograms were provided ha B. C Johnson, Lawrence Livermore Laboratory. Livermore, CA

109. P. W. Smith. "Mode-Locking of Lasers," Proc. IEEE 58, 1312 (1970).

110. D. J. Kuizenga and A. E. Siegman. "FM and AM Mode-Locking of the Homogentous l.alser-Parts I \& 11," IEEE J. Quan. Elear. QE-6, 673 (1970).

111. A. E. Siegman and D. J. Kuizenga, "Active Mode-Coupling Phenomena in Pulsed and Contınusus Lasers," Opt. Elect. 6, 63 (1974).

112. Laser Program Annual Report. Lawrence Livermore Laboratory, Livermore. CA. UCRL-50021-77 (1978). p. 2-210; and UCRL-50021-78 (1979).

113. W. E. Martin, B. C. Johnson, and K. R. Guinn, "Pulse Shaping for Laser Fusion," in IEEE/OSA Cin!!. on Laser Engineering and Application. Washington. D.C., 1977, p. 53.

114. D. J. Kuizenga and W. E. Martin. "Argus Oscillator and Pulse Stacker," Laser Program Annual Repori, Lawrence Livermore Laboratory, Livermore, CA, UCRL-50021-77 (1978), p. 2-219.

115. These examples of coherent pulse shortening and stretching were obtuiried by J. E. Murray. For detusk see Laser Program Anmual Report, Lawrence Livermore Laboratory, Livermore, CA, UCRL-50021-77 (1978), p. 2-210; and UCRL-50021-78 (1979), p. 7-11.

116. C. Brecher, L. A. Risenberg, and M. J. Weber, "Line-Narrowed Fluorescence Spectra and SiteDependent Transition Probabilities of $\mathrm{Nd}^{3+}$ in Oxide and Fluoride Glasses," Phys. Rev. E 18, 5799 (1978).

117. J. C. Almasi, J. P. Chernock, W. S. Martin, and K. Tomiyasu, "Face Pumped Laser," General Electric Report to the Office of Naval Research, AD-467 468/5 ST (May 1966).

118. J. E. Swain, R. E. Kidder, K. Pettipiece, F. Rainer, E. D. Baird, and B. Loth, "Large-Aperture Disk Laser System," J. Appl. Phys. 40, 3973 (1969).

119. W. Koechner, Solid State Laser Engineering (Springer-Verlag, NY, 1976).

120. G. J. Linford, R. A. Saroyan, J. B. Trenholme, and M. J. Weber, "Measurements and Modeling of Gain Coefficients for Neodymium Laser Giasses," IEEE J. Quant. Elect. QE-15, 510 (1979).

121. A. J. Beaulieu, "Transversely Excited Atmospheric Pressure $\mathrm{CO}_{2}$ Lasers," Appl. Phys. Lett. 16, 504 (1970). 
122. R. Dumanchin and J. Roced-Serra. "Augmentation de L'energic at de la Puissance Fourne Par Unite de Volume dans un Laser a $\mathrm{CO}_{2}$ en Regime Pulse," Compt. Rend. A cad. Sci. 269 (1969).

123. J. D. Daugherty, E. R. Pugh, and D. H. Douglas-Hamilton, "h Stable, Scalable High Pressure Gas Discharge as Applied to the High Pressure CO, Laser," Bull. Am. Phys. Soc. 17, 399 (1972).

124. C. A. Fenstermacher, M. J. " Hler, W. T. Leland, and K. Boyer, "Electron Beam Controlled Electrical Discharge as a Method of Pumping Large Volumes of $\mathrm{CO}_{2}$ Laser Media al High Pressure." Appl. Phy's. Lell. 20, 56 (1972).

125. N. G. Basov, "Gas Lasers at High Pressures," JETP Lett. 14, 285 (1971).

126. For a review sec J. D. Daugherty, "Electron Bean. Innized Lasers," in Principles of Laser Plasma, G. Bekefe, Ed. (John Wiley \& Sons, NY, 1976), F. 369.

127. J. M. Auerbuch, iN. C. Holmes, J. T. Hunt, and G. J. Linford, "Closure Phenomena in Pinhules Irradialed hy Nd Laser Pulses," Appl. Opl, 18, 2495 (1979).

128. L. J. Aplet and J. W. Carson, "A Faradiy Effect Optical Isolator," Appl. Opt. 3, 544 (1974).

129. K. Eidman, P. Sachsenmaic, H. Salzmann, and R. Sigel, "Optical Isolaturs for High Power Giant Pulse Lasers," J. Phy's. E 5, 56 (1972).

130. For a revew and history of electro-optic switchess see I. P. Kaminow. An Introduction an Electro-Optic Devices (Academic Press, NY, 1974).

131. M. A. Summers and B. C. Johnson, Design and Performance of Large Aperture KD*P Pockels Cells. Lawrence Livermure Laboratory, Livermore, CA. UCRL-79952 (1977).

132. M. J. Weber, "Optical Materials for Neodymium Fusion Latsers," reprinted from Critical Materials Prohlems in Energy Production (Academic Press. NY. 1976). p. 261.

133. For detaited descriptions of laser and target optical diagnostics, see the Amnual Reports from the laboratories involved in laser plasmia interaction work. These laboratories include: CEA Limeil, France: KMS Fusion, Inc., Ann Arbor, MI: Naval Research Laboratory, Washinglon, DC: Osii..al University, Osaka, Japan: Sandia Laboratories, Albuquerque, NM: the Lebedev Institute, Moscon. USSR: the Max Planck Institute for Plasma Physics, Garching, Germany; the Rutherford Laboratory. Didcot, England; Lawrence Livermore Laboratory, Livermore, CA; Los Alamos Scientific Laboratory. Los Alamos, NM; University of Rochester, Laboratory for Laser Energetics, Rochester, NY.

134. The diagnostic techniques used to verify the initial beam-target relationship in the Livermore Luser Program were reviewed in the Laser Program Annual Report, Lawrence Livermore Lahoratory, Livermore, CA, UCRL-50021.75 (1976), p. 369.

135. In a laser system operating safely within its propagation limits, and in which the amplifiers are uniformiv pumped, a "square-profile" laser pulse and a low-level cw laser beam will propagate in virtually an identical manner. However, if the amplifier pumping characteristics are significantly nonuniform, then thermal lensing will occur due to nonuniform heat deposition, and the focal properties of the pulsed beam will differ from those of the $\mathrm{cw}$ alignment beam. Examples of pump-induced distortion are found in G. J. Linford, H. H. Chau, J. A. Glaze, C. B. Layne, and F. Rainer, "Pump-Induced Optical Distortions in Disk Amplifier Modules: Holographic and Interferometric Measurements," Appl. Opt. 14, 3057 (1975).

136. J. Petruzzi and C. E. Violet, Positioning Alignment Balls at the Focal Point of a Lens. I: Preliminary Measurements. Lawrence Livermore Laboratory, Livermore, CA, UClR-749 (1974); and C. E. Violet and J. Petruzzi, Positioning Alignment Balls at the Focal Point of a Lens. II: Two Beam Mock Up. Lawrence Livermore Laboratory, Liverm:cre, CA, UCIR-859 (1975).

137. J. B. Trenholme, E. S. Bliss, J. L. Emmett, J. A. Glaze, T. J. Gilmartin, R. O. Godwin, W. F. Hagen, J. F. Holzrichter, G. J. Linford, W. W. Simmons, and D. R. Speck, "Fusion Laser Development at the Lawrence Livermore Laboratory," in Laser Interactions and Related Plasma Phenomena, J. Schwartz and H. Hora, Eds. (Plenum Press, NY, 1977), Vol. 4A, p. 1.

138. N. G. Basov, O. N. Krokhin, Yu. A. Mikhailov, G. V. Sklizkov, and S. I. Fedotov, "Powerful 12Channel Laser Installation 'Delfin' for Spherical Heating of Thermonuclear Targets," in Laser Interaclion and Related Plasma Phenomena, ibid. 
139. W. Sekd, J. Soures, O. Lew is, J. Bunkenherg. D. Broun, S. Jacobs, G. Mourou, and J. Zimmerman. "Design and Performance Charatteristles of a High Power Phosphate Glass Laser System." Appl. Opt. 19.: .3 (1980). pp. 409-419, also avallable as Laboratory for Laser Energetics Report 6 ). Universily of Ruchester, Rochester, NY.

140. S. Singer, "CO2 Laser Systems for Fusion Experiments." in Laser Interacrions and Related Plasma Phenomena (Plenum Press. NY, 1977). Vol. 4A. p. 121.

$1+1$ T. F. Stratton, "CO 2 Short-Pulse Laser Technology," in Lectures on High Power Gas Lasers at miternational College' of Applied Phisios on Capri. Septemher 1975 (Institute of Physies, London, Insi. Phys Conf. Ser. No, 29, E. R. Pike. Ed., 1976). p. 284.

142. The ideas for multipass-laser-systen architecture have been present for mans sears. One of the carlest examples was the long-path laser constructed at the Lawrence Livermore Laboratory in 1965-68. A deseription can be found in S. W. Mead, R. E. Kidder, J. E. Swaln, F. Ranter, and J. Patru/s!, "Preliminary Measurements of X-ray and Neutron Emission from Laser Produced Plasmias," Appl. Opt. 11, 345 (1972). I1 was not completely sucessful because the very long propagalion paths led to severe beam distortion. Relay amaging and hetter understanding of selffocusing make these systems. feasible loday. For a review of recent ideals on multipass systems, nE. CP\&D Imerim Repurt - Shuva Vova, T. J. Gilmartin and C. W. Meier, Eds., Lilwrence Livermore Laborators, Livermore. CA. Mist. Report 107 (1977), pp. 2-161.

143. W. F. Hatgen, "Multipass Laser Systems-A nalysis and Optimization," Laser Prugram , Anmual Repurt. Lawrence Livermore Liborators, Livermore, CA, UCRL-50021-77 (1978), p. 2-224.

14. This geometry has been employed on the laser systems at KMS Fusion, Inc., Ann Arbor. MI.

145. M. D. Montgomery, R. L. Carlson, D. E. Cisperson, S. J. Cauthlewski, J. F. Figreira, R. F. Haglund Jr., J. S. Ladish. A. V. Nowak, and S. Singer. "Stahilication of High-Gain Multupass Pawer Amplifiers Using Saturable Ahsorhers: Experience un the LASL Eight Bean System." Appl Phr. Le'lt 32. 324 (1978).

146. The optimization of Nd:laser systems to obtuin the greatest performance dollur cost is described in the Laser Program Anmal Reporl. Lawrence Livermore Lahoratory. Livermore, CA. UCRL-50021-74 to 78 (1975-1979). For a concise review of glass-laser optimization, see the article by J. B. Trenholne el al., "Laser System Design-Shiva Nova," in Nova. T. J. Gilmartin and C. W. Meier, Eus., Lawrence Livermore Laboratory, Livermore, CA, Misc. Report 107 (1977).

147. The isofluence laser concept is reviewed in the Nova CP\&D Reports, Lawrence Livermore Laboratory. Livermore, CA, Misc. Reports 2242 (1976), 107 (1977), and 111 (1978).

148. The costs of Nd:glass laser system components are reviewed in the Nova CP\&D Reports, ibid.

149. J. L. Emmetl, "Approaches to Extremely High-Power Laser Systems," in Topical Meeting on Inertial Confinement Fusion, Feb. 7-9. 1978, San Diego, CA. 